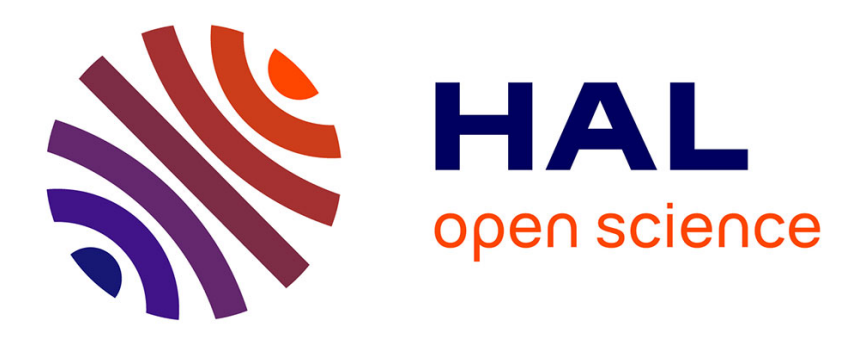

\title{
Isomonodromic deformation of Lamé connections, Painlevé VI equation and Okamoto symetry
}

Frank Loray

\section{To cite this version:}

Frank Loray. Isomonodromic deformation of Lamé connections, Painlevé VI equation and Okamoto symetry. Izvestiya: Mathematics, 2016, 80 (1), pp.113-116. 10.1070/IM8310 . hal-01075998

\author{
HAL Id: hal-01075998 \\ https://hal.science/hal-01075998
}

Submitted on 20 Oct 2014

HAL is a multi-disciplinary open access archive for the deposit and dissemination of scientific research documents, whether they are published or not. The documents may come from teaching and research institutions in France or abroad, or from public or private research centers.
L'archive ouverte pluridisciplinaire HAL, est destinée au dépôt et à la diffusion de documents scientifiques de niveau recherche, publiés ou non, émanant des établissements d'enseignement et de recherche français ou étrangers, des laboratoires publics ou privés. 


\title{
ISOMONODROMIC DEFORMATION OF LAMÉ CONNECTIONS, PAINLEVÉ VI EQUATION AND OKAMOTO SYMETRY
}

\author{
FRANK LORAY \\ In the memory of Andrei Bolibrukh
}

\begin{abstract}
A Lamé connection is a logarithmic sl $(2, \mathbb{C})$-connection $(E, \nabla)$ over an elliptic curve $X:\left\{y^{2}=x(x-1)(x-t)\right\}, t \neq 0,1$, having a single pole at infinity. When this connection is irreducible, we show that it is invariant by the standart involution and can be pushed down as a logarithmic sl $(2, \mathbb{C})$-connection over $\mathbb{P}^{1}$ with poles at $0,1, t$ and $\infty$. Therefore, the isomonodromic deformation $\left(E_{t}, \nabla_{t}\right)$ of an irreducible Lamé connection, when the elliptic curve $X_{t}$ varry in the Legendre family, is parametrized by a solution $q(t)$ of the Painlevé VI differential equation $P_{V I}$. We compute the variation of the underlying vector bundle $E_{t}$ along the deformation via Tu moduli map: it is given by another solution $\tilde{q}(t)$ of $P_{V I}$ equation related to $q(t)$ by the Okamoto symetry $s_{2} s_{1} s_{2}$ (NoumiYamada notation). Motivated by the Riemann-Hilbert problem for the classical Lamé equation, the question whether Painlevé transcendents do have poles is raised. Those results were partially announced in $[23]$.
\end{abstract}

\section{Contents}

Motivations 2

1. Result 5

2. Our main construction: elliptic pull-back 8

3. Computing the vector bundle of an elliptic pull-back 11

3.1. Proiective bundles 12

3.2. Ruled surfaces and elliptic pull-back 13

3.3. Elliptic ruled surfaces and elementary transformations 14

4. Isomonodromic deformations and the Painlevé VI equation 18

4.1. Isomonodromic deformations and flat connections 18

4.2. Painlevé VI equation and fuchsian equations 19

4.3. Painlevé VI equation and fuchsian svstems 20

4.4. The vector bundle of an elliptic pull-back 22

Date: October 18, 2014. 
5. The geometry of Painlevé VI equations 23

5.1. Okamoto's space of initial conditions 24

5.2. Projective structures and Riccati foliations 25

5.3. Riccati foliations on $\mathbb{F}_{2}$

$\begin{array}{lll}\text { 5.4. Riccati foliations on } \mathbb{F}_{1} & 29\end{array}$

5.5. Riccati foliations on $\mathbb{F}_{0}=\mathbb{P}^{1} \times \mathbb{P}^{1} \quad 29$

5.6. The moduli space $\mathcal{M}_{+}^{\kappa} \quad 30$

5.7. The moduli space $\mathcal{M}_{+}^{\boldsymbol{\theta}}$ of $\mathrm{sl}(2, \mathbb{C})$-connections 34

5.8. Okamoto symetries 35

5.9. Special configurations 38

5.10. Bolibrukh-Heu transversality 39

6. Lamé connections 40

6.1. Riemann-Hilbert correspondence 41

6.2. Fricke moduli space $\quad 42$

6.3. Resonant cases 44

6.4. Irreducible Lamé connections are elliptic pull-back 48

7. Proof of Theorem 1150

8. Flat logarithmic sl $(2, \mathbb{C})$-connections 50

8.1. Polar divisor 51

8.2. Flatness and monodromv representation 51

8.3. Flat logarithmic connections $\quad 52$

8.4. Trace and twist 53

8.5. $\mathrm{sl}(2, \mathbb{C})$-connections 53

8.6. Riemann-Hilbert correspondence 54

8.7. Reducible gl $(2, \mathbb{C})$-connections $\quad 54$

8.8. Projective sl(2. $\mathbb{C}$ )-connections and Riccati foliation $\quad 55$

8.9. Stability of bundle and connections 57

8.10. Meromorphic and elementary gauge tranformations $\quad 57$ References

\section{Motivations}

The classical Lamé equation (here in Legendre form)

(1) $\frac{d^{2} u}{d x^{2}}+\frac{1}{2}\left(\frac{1}{x}+\frac{1}{x-1}+\frac{1}{x-t}\right) \frac{d u}{d x}+\frac{c-n(n+1) x}{4 x(x-1)(x-t)} u=0$,

$t, n, c \in \mathbb{C}, t \neq 0,1$, defines a projective structure on the elliptic curve

$$
X:\left\{y^{2}=x(x-1)(x-t)\right\}, \quad t \in \mathbb{C}-\{0,1\}
$$


having a fuchsian singularity at the point $\omega_{\infty}$ at infinity. On the affine part of the curve, projective charts are local determinations of

$$
\phi: X-\left\{\omega_{\infty}\right\} \rightarrow \mathbb{P}^{1} ;(x, y) \mapsto \frac{u_{1}(x)}{u_{2}(x)}
$$

where $u_{1}$ and $u_{2}$ range over independant solutions of (11). Setting $\vartheta:=$ $2 n$, there is a local coordinate $z$ at $\omega_{\infty}$ such that one of the projective charts around this point is given by

- $\phi=z^{\vartheta}$ when $\vartheta \notin \mathbb{Z}$,

- $\phi=z^{m}$ or $\phi=\frac{1}{z^{m}}+\log (z)$ when $\vartheta= \pm m, m \in \mathbb{Z}_{>0}$,

- $\phi=z$ (regular) when $\vartheta=0$.

The monodromy of any projective chart $\phi=\frac{u_{1}}{u_{2}}$ after analytic continuation along any loop $\gamma$ is given by $\rho(\gamma) \circ \phi$ where

$$
\rho: \pi_{1}\left(X-\left\{\omega_{\infty}\right\}\right) \rightarrow \operatorname{PGL}(2, \mathbb{C})
$$

is the projective monodromy representation of (11) computed in the basis $\left(u_{1}, u_{2}\right)$. The following natural question goes back to Poincaré (see [36]): which topological representation

$$
\left.\rho: \pi_{1} \text { (once punctured torus }\right) \rightarrow \operatorname{PGL}(2, \mathbb{C})
$$

occurs as the monodromy of a fuchsian projective structure?

Any fuchsian projective structure on the once punctured torus (i.e. having moderate growth at the puncture) is of the form above: the parameter $t$ stands for the underlying complex structure, $n$ (or $\vartheta=2 n+1$ ) for the fuchsian type of the puncture and $c$ is the accessory parameter. The number of parameters fits with the dimension of the space of those topological representations up to conjugacy (see [13]). One thus expects that a generic representation should be the monodromy of some Lamé equation. The similar question for regular projective structures on complete curves have been answered only recently in [12] by pants decomposition and gluing methods. Our initial aim, originating the present work, was to use isomonodromy method in order to answer the Lamé case as a test; as we shall see, we actually reduce the above question to the existence of poles for Painlevé VI transcendents, which looks difficult, of a different nature though.

The Lamé equation may be viewed as a logarithmic sl $(2, \mathbb{C})$-connection on the trivial vector bundle $\mathcal{O} \oplus \mathcal{O}$ over the elliptic curve $X$, having a single pole at $\omega_{\infty}$ : any eigenvector of the residual matrix connection at $\omega_{\infty}$ provides a cyclic vector going back to the scalar elliptic form. On the other hand, the Riemann-Hilbert correspondence asserts that any representation

$$
\rho: \pi_{1}\left(X-\left\{\omega_{\infty}\right\}\right) \rightarrow \mathrm{SL}(2, \mathbb{C})
$$


is the monodromy of a logarithmic sl $(2, \mathbb{C})$-connection $\nabla$ on some rank 2 vector bundle $E$ over $X$, having a single pole at $\omega_{\infty}$. Our initial question becomes: for a given topological representation (3), can we choose the complex structure $X$ in such a way that the realizing connection $(E, \nabla)$ is defined on the trivial bundle? Now, the question perfectly fits into the setting of isomonodromic deformations.

Starting with a "Lamé connection" $\left(E_{0}, \nabla_{0}\right)$ on $X_{t_{0}}$, consider its isomonodromic deformation $\left(E_{t}, \nabla_{t}\right)$ when the complex structure of the curve $X_{t}$ varry; here, the parameter $t$ of the deformation has to be considered in the universal cover $T \simeq \mathbb{H} \rightarrow \mathbb{P}^{1}-\{0,1, \infty\}$, the Teichmüller space of the once punctured torus. When the pole of the Lamé connection is not apparent, this unique deformation is characterized by the fact that its monodromy representation is locally constant. Equivalently, the deformation is induced by the unique logarithmic integrable connection on the universal curve $\boldsymbol{X} \rightarrow T$ having a single pole along the section $t \mapsto \omega_{\infty} \in X_{t}$ whose restriction to the initial fiber $X_{t_{0}}$ coincides with $\left(E_{0}, \nabla_{0}\right)$. Our initial question becomes: for which connection/representation can we insure that the underlying vector bundle $E_{t}$ becomes trivial for some convenient parameter $t$ along the isomonodromic deformation of a Lamé connection?

In this paper, we will compute the variation of the vector bundle $E_{t}$ along the isomonodromic deformation of a Lamé connection and show that it is given by a solution of the Painlevé VI equation for some convenient parameter; the bundle $E_{t}$ becomes then trivial only when the Painlevé transcendent has a pole (outside 0,1 and $\infty$ ). We are finally led to the following question which seems widely open: do Painlevé transcendents have poles?

Actually, much more interesting is the similar question for regular connections over genus $g>1$ curves. Following [14, regular projective structures correspond there to regular connections on the maximally unstable undecomposable $\mathrm{SL}(2, \mathbb{C})$-bundle (a sl $(2, \mathbb{C})$-oper in the sense of [3], §2.7). The main result of [12] can be rephrased as follows: the special bundle above occur along the isomonodromic deformation if, and only if, the monodromy is neither reducible, nor in $\mathrm{SU}(2, \mathbb{C})$ (up to $\mathrm{SL}(2, \mathbb{C})$-conjugacy). Can we prove this directly by computing the variation of the bundle?

Another interseting question when $g>1$ is whether a given topological representation $\pi_{1}\left(X^{t o p}\right) \rightarrow \mathrm{SL}(2, \mathbb{C})$ can be realized as the monodromy of a connection on the trivial bundle, for a convenient choice of complex structure $X$ ? In the case the image $\Gamma$ is a discrete subgroup, 
this provides an embedding $X \rightarrow \mathrm{SL}(2, \mathbb{C}) / \Gamma$ : the fundamental matrix of the associate system defines an equivariant map $\tilde{X} \rightarrow \operatorname{SL}(2, \mathbb{C})$ (where $\tilde{X} \rightarrow X$ the universal cover). The existence of compact curves in quotients of $\operatorname{SL}(2, \mathbb{C})$ is still an open problem. The isomonodromic approach gather into the same geometrical framework questions arising from various contexts.

\section{Result}

Isomonodromic deformations of meromorphic connections have been extensively studied over the Riemann sphere (see [27, 34]). In this situation, the underlying vector bundle is constant on a Zariski open subset of the parameter space (see [5]) allowing an explicit computation of the isomonodromy condition, namely the Schlesinger equations; Painlevé equations arise after further reduction in the simplest case: rank 2 with 4 poles. In order to observe some continuous deformation of the underlying bundle, one has to switch to connections over genus $g>0$ curves. The simplest non trivial case, namely regular rank 1 connections over an elliptic curve, has been considered in [17, 25]: there, it was observed that the variation of the underlying line bundle along an isomonodromic deformation is a Painlevé transcendent. In this paper, we study the next case in difficulty, namely rank 2 logarithmic connections over elliptic curves with a single pole.

Throughout this work, a Lamé connection consists in the data $(E, \nabla)$ of a rank 2 locally trivial holomorphic vector bundle $E$ over an elliptic curve belonging to the Legendre family:

$$
X:\left\{y^{2}=x(x-1)(x-t)\right\}, \quad t \in \mathbb{C}-\{0,1\}
$$

equipped with a logarithmic and trace free connection $\nabla$ having (at most) a single pole at the point $\omega_{\infty}$ at infinity:

$$
\nabla: E \rightarrow E \otimes \Omega_{X}^{1}\left(\left[\omega_{\infty}\right]\right), \quad \operatorname{det}(E)=\mathcal{O}_{X}, \quad \operatorname{trace}(\nabla)=d: \mathcal{O}_{X} \rightarrow \Omega_{X}^{1}
$$

(here, we identify vector bundles $E$ and $\Omega$ with the corresponding sheaves of holomorphic sections). Up to now, such a connection $(E, \nabla)$ will be considered up to holomorphic bundle isomorphism. The exponent $\vartheta \in \mathbb{C}$ of $(E, \nabla)$, defined up to a sign, is the difference between the eigenvalues $\pm \frac{\vartheta}{2}$ of the residual matrix connection at $\omega_{\infty}$.

The underlying vector bundle of a Lamé connection has trivial determinant because of the trace free condition. Following Atiyah [2], almost all rank 2 vector bundles on $X$ with trivial determinant are decomposable, i.e. of the form

$$
E=L \oplus L^{-1} \quad \text { with } \quad L \in \operatorname{Pic}(X) ;
$$


to complete the list, one has to add 4 extra bundles $E_{i}, i=0,1, t, \infty$. Semistable bundles are those decomposable ones with $L \in \operatorname{Pic}^{0}(X)$, i.e. of the form

$$
E=L \oplus L^{-1} \quad \text { with } \quad L=\mathcal{O}_{X}\left([\omega]-\left[\omega_{\infty}\right]\right), \omega=(x, y) \in X
$$

together with the 4 undecomposable ones above. The corresponding moduli space is $\mathbb{P}^{1}$ (see [39]) with quotient map given by

$$
\lambda:\left\{\begin{array}{cc}
E \mapsto x & \text { under notation of (6) } \\
E_{i} \mapsto i & i=0,1, t, \infty
\end{array}\right.
$$

If we denote by $\omega_{i}=(i, 0) \in X$ the 2 -torsion points, $i=0,1, t, \infty$, then $E_{i}$ is in fact the unique non trivial extension

$$
0 \rightarrow L_{i} \rightarrow E_{i} \rightarrow L_{i} \rightarrow 0, \quad \text { with } L_{i}=\mathcal{O}\left(\left[\omega_{i}\right]-\left[\omega_{\infty}\right]\right)
$$

the moduli map $\lambda$ identifies $E_{i}$ with the trivial extension $L_{i} \oplus L_{i}$. In particular, the point $\lambda=\infty$ corresponds to both the trivial vector bundle and $E_{\infty}$.

The isomonodromic deformation of a Lamé connection is defined as follows. Consider the universal cover $T \rightarrow \mathbb{P}^{1}-\{0,1, \infty\}$, namely the Teichmüller space of the punctured torus, and the universal Legendre curve $\boldsymbol{X} \rightarrow T$ over this parameter space: the fiber $X_{t}$ at some point $t$ is precisely the curve $\left\{y^{2}=x(x-1)(x-t)\right\}$ (by abuse of notation, we denote by $t$ a point of $T$ and its projection on $\left.\mathbb{P}^{1}\right)$. The point $\omega_{\infty}$ at infinity defines a section $T \rightarrow \boldsymbol{X}$ of this fibration. Starting from a Lamé connection $\left(E_{0}, \nabla_{0}\right)$ over $X_{t_{0}}$, there is a unique logarithmic flat connection $(E, \nabla)$ over the total space $\boldsymbol{X}$, having the section $\omega_{\infty}$ as polar set and inducing the initial connection $\left(E_{0}, \nabla_{0}\right)$ on $X_{t_{0}}$ (see [10, 27, 16]). The deformation $t \mapsto\left(E_{t}, \nabla_{t}\right)$ induced over the family $t \mapsto$ $X_{t}$ is the isomonodromic deformation of $\left(E_{0}, \nabla_{0}\right)$. When the pole of $\nabla_{0}$ is not an apparent singular point (i.e. having local monodromy $\pm I$ ), then $t \mapsto\left(E_{t}, \nabla_{t}\right)$ is precisely the unique deformation having constant monodromy representation. The exponent $\vartheta$ of the Lamé connection is constant along such a deformation. Finally, one can talk about the variation of the underlying vector bundle $E_{t}$ along the deformation just by considering the moduli map $t \mapsto \lambda\left(E_{t}\right)$ defined above. Our result is

Theorem 1. Let $\left(E_{t}, \nabla_{t}\right)$ be the isomonodromic deformation of an irreducible Lamé connection. Then, for a Zariski open subset of the parameter space $T$, the underlying vector bundle $E_{t}$ is semistable (see [16]) and its $T u$ invariant $t \mapsto \lambda\left(E_{t}\right) \in \mathbb{P}^{1}$ defined by (7) is solution of 
the Painlevé VI differential equation

$$
\begin{aligned}
& \frac{d^{2} \lambda}{d t^{2}}=\frac{1}{2}\left(\frac{1}{\lambda}+\frac{1}{\lambda-1}+\frac{1}{\lambda-t}\right)\left(\frac{d \lambda}{d t}\right)^{2}-\left(\frac{1}{t}+\frac{1}{t-1}+\frac{1}{\lambda-t}\right)\left(\frac{d \lambda}{d t}\right) \\
& \quad+\frac{\lambda(\lambda-1)(\lambda-t)}{t^{2}(t-1)^{2}}\left(\frac{\kappa_{\infty}^{2}}{2}-\frac{\kappa_{0}^{2}}{2} \frac{t}{\lambda^{2}}+\frac{\kappa_{1}^{2}}{2} \frac{t-1}{(\lambda-1)^{2}}+\frac{1-\kappa_{t}^{2}}{2} \frac{t(t-1)}{(\lambda-t)^{2}}\right) .
\end{aligned}
$$

with parameter $\left(\kappa_{0}, \kappa_{1}, \kappa_{t}, \kappa_{\infty}\right)=\left(\frac{\vartheta}{4}, \frac{\vartheta}{4}, \frac{\vartheta}{4}, \frac{\vartheta}{4}\right)$ where $\vartheta$ is the exponent of the Lamé connection.

By "Zariski open", we just mean that exceptional values of $t$ form a discrete subset of the parameter space $T$. This actually directly follows from [16].

It is already known that isomonodromic deformation of (generic) Lamé connections are parametrized by Painlevé VI equation with parameters specified above: in [21, 22], isomonodromic deformation equations are directly computed on the elliptic curve, and the elliptic form of Painlevé VI equation (see 28]) is recognized.

Our approach of this result is quite different. We first prove, using the Riemann-Hilbert correspondence, that any irreducible Lamé connection can be pushed down, via the 2 -fold cover $X \rightarrow \mathbb{P}^{1}$ as a logarithmic rank 2 connection over $\mathbb{P}^{1}$ with 4 poles, namely at the ramification values $0,1, t$ and $\infty$. We are back to the classical case of Fuchs: the isomonodromic deformation is parametrized by a solution, say $q(t)$, of the Painlevé VI equation with parameter $\left(\kappa_{0}, \kappa_{1}, \kappa_{t}, \kappa_{\infty}\right)=$ $\left(\frac{1}{2}, \frac{1}{2}, \frac{1}{2}, \frac{\vartheta-1}{2}\right)$. This already explains why Painlevé VI equation arises in the Lamé case; so far, no computation is needed. Elementary birational geometry is used to go back to the initial deformation $\left(E_{t}, \nabla_{t}\right)$ and the moduli $\lambda$ of the vector bundle $E_{t}$ can be computed by means of $q(t)$ : we recognize in $\lambda\left(E_{t}\right)$ the image of $q(t)$ by the Okamoto symetry $s_{1} s_{2} s_{1}$ (see [30]). This automatically implies that $\lambda\left(E_{t}\right)$ is also solution of the Painlevé VI equation, but with new parameter $\left(\frac{\vartheta}{4}, \frac{\vartheta}{4}, \frac{\vartheta}{4}, \frac{\vartheta}{4}\right)$.

A similar statement holds for the classical Painlevé VI setting (logarithmic $\operatorname{sl}(2, \mathbb{C})$-connections over $\mathbb{P}^{1}$ with 4 poles), when considering the parabolic bundle defined by eigendirections of the residual matrix of the connection (see [1, 26]).

More generally, we can start with the isomonodromic deformation of a rank 2 logarithmic connection with 4 poles over $\mathbb{P}^{1}$, parametrized by any Painlevé VI transcendent $q(t)$. Then one can lift-up conveniently the deformation over the Legendre elliptic curve $X_{t}$ as a rank 2 logarithmic connection with poles at the ramification points $\omega_{i}$ in such a way that the moduli $\lambda\left(E_{t}\right)$ of the vector bundle is the Okamoto symetric $s_{1} s_{2} s_{1}$ of $q(t)$; this provides a new geometric interpretation of this strange symetry. We thus obtain in a natural way an isomonodromic deformation problem (a Lax pair) for the general elliptic form of the 
Painlevé VI equation, just by considering those rank 2 and trace free logarithmic connection over $X_{t}$ having poles at the 2-order points $\omega_{i}$ that moreover commute with the elliptic involution $(x, y) \mapsto(x,-y)$; this has been also considered in [41].

When we set $\vartheta=0$, all Lamé connections with vanishing exponent are reducible, but those regular ones can still be pushed down to $\mathbb{P}^{1}$ : our result remains valid in this case and we retrieve the

Corollary 2 ([17, 25]). Let $t \mapsto\left(L_{t}, \nabla_{t}\right)$ be the isomonodromic deformation of a regular rank 1 connection on the Legendre deformation $X_{t}$ and let $E_{t}=\mathcal{O}\left([\omega(t)]-\left[\omega_{\infty}\right]\right)$ be the underlying line bundle, $\omega(t)=(x(t), y(t)) \in X_{t}$. Then $t \mapsto x(t)$ is solution of Painlevé VI equation with parameters $\left(\kappa_{0}, \kappa_{1}, \kappa_{t}, \kappa_{\infty}\right)=(0,0,0,0)$.

One can explicitely compute, in this case, the variation of the line bundle $L_{t}$ by means of elliptic functions and retrieve the

Corollary 3 (Picard [35], see [29]). The general solution of Painlevé $V I$ equation with parameters $\left(\kappa_{0}, \kappa_{1}, \kappa_{t}, \kappa_{\infty}\right)=(0,0,0,0)$ is given by

$$
t \mapsto x(t) \quad \text { where } \quad(x(t), y(t)):=\pi\left(c_{0} \cdot \omega_{0}+c_{1} \cdot \omega_{1}\right), c_{0}, c_{1} \in \mathbb{C}
$$

where $\pi: \mathbb{C} \rightarrow X_{t}$ is the universal cover and $\omega_{i}(t)$, half-periods of $X_{t}$.

The Painlevé transcendents of Corollary 3 have poles if, and only if, either $c_{0}$ or $c_{1}$ is not real.

\section{OUR MAIN CONSTRUCTION: ELLIPTIC PULL-BACK}

Here, we construct Lamé connections by lifting on the elliptic twofold cover $\pi: X \rightarrow \mathbb{P}^{1}$ certain $\mathrm{sl}(2, \mathbb{C})$-connections having logarithmic poles at the critical values of $\pi$. Later, we will prove that all irreducible Lamé connections can be obtained by this way; this will be used to parametrize their isomonodromic deformation by means of Painlevé VI solutions in an explicit way.

Let us fix exponents $\boldsymbol{\theta}=\left(\theta_{0}, \theta_{1}, \theta_{t}, \theta_{\infty}\right)$ and consider a logarithmic $\operatorname{sl}(2, \mathbb{C})$-connection $(E, \nabla)$ over $\mathbb{P}^{1}$ with poles at $0,1, t$ and $\infty$ and prescribed exponents: eigenvalues of the residual matrix at $i$ are $\pm \frac{\theta_{i}}{2}$, for $i=0,1, t, \infty$. Such a connection will be called a Heun connection.

The important data that will be used later is the parabolic structure $\boldsymbol{l}=\left(l_{0}, l_{1}, l_{t}, l_{\infty}\right)$ defined by the eigenline $l_{i} \in \mathbb{P}\left(\left.E\right|_{i}\right)$ of the residue of $\nabla$ at $i$ with respect to the eigenvalue $-\frac{\theta_{i}}{2}$, for $i=0,1, t, \infty$. If $\nabla$ do have pole at each $\omega_{i}$, the parabolic structure is perfectly well defined by the connection and the choice of exponents $\theta_{i}$ (they are defined up to a sign). However, it is important to allow non singular points in our construction if we want to fit with the usual Painlevé VI 
phase space (see [19]): when $\theta_{i}=0$ and the corresponding point is non singular, then any line $l_{i} \in \mathbb{P}\left(\left.E\right|_{i}\right)$ is an eigenline and we have to choose one for our construction. We call parabolic Heun connection with parameter $\boldsymbol{\theta}$ the data $(E, \nabla, \boldsymbol{l})$ with above properties.

Example 4. When $E$ is the trivial bundle, then $\nabla$ is defined by a fuchsian system

$$
\frac{d Y}{d x}=\left(\frac{A_{0}}{x}+\frac{A_{1}}{x-1}+\frac{A_{t}}{x-t}\right) Y, \quad A_{i} \in \operatorname{sl}(2, \mathbb{C}) .
$$

The residual matrix at $x=i$ is given by $A_{i}$ for $i=0,1, t, \infty$ where $A_{\infty}$ is defined by

$$
A_{0}+A_{1}+A_{t}+A_{\infty}=0 .
$$

Exponent restrictions are given by $\operatorname{det}\left(A_{i}\right)=-\frac{\theta_{i}^{2}}{4}$ and the parabolic structure, by $l_{i}=\operatorname{ker}\left(A_{i}+\frac{\theta_{i}}{2} I\right)$.

In order to motivate the following construction, let us indicate that for special exponents

$$
\boldsymbol{\theta}=\left(\frac{1}{2}, \frac{1}{2}, \frac{1}{2}, \frac{1}{2}+\frac{\vartheta}{2}\right)
$$

it will provide a Lamé connection with exponent $\vartheta$ at infinity.

Step 1 : We pull-back the connection $(E, \nabla)$ on the elliptic cover

$$
\pi: X \rightarrow \mathbb{P}^{1} ;(x, y) \mapsto x .
$$

We obtain a logarithmic $\operatorname{sl}(2, \mathbb{C})$-connection on $X$

$$
(\tilde{E}, \tilde{\nabla}):=\pi^{*}(E, \nabla)
$$

with poles at the ramification points $\omega_{0}, \omega_{1}, \omega_{t}$ and $\omega_{\infty}$ and twice the initial exponents $2 \theta_{i}$. The parabolic structure $\tilde{\boldsymbol{l}}:=\pi^{*} \boldsymbol{l}$ corresponds to eigenlines with respect to eigenvalues $-\theta_{i}$. The point $i$ is parabolic (resp. non singular) for $\nabla$ if, and only if, the point $\omega_{i}$ is so for $\tilde{\nabla}$. We already note that, for exponents $\theta_{i}=\frac{1}{2}$ for $i=0,1, t$, the corresponding singular points $\omega_{i}$ of $(\tilde{E}, \tilde{\nabla})$ are projectively apparent, i.e. having $-I$ local monodromy. The next two steps will clear them off.

Remark that we could have choosen an initial connection on $\mathbb{P}^{1}$ with a single pole at $\infty$ so that its lifting is of Lamé type; but the monodromy would then be trivial in this case, and the Lamé connection "very reducible".

Step 2 : We make a convenient birational bundle modification

$$
\phi: \tilde{E} \rightarrow \tilde{E}^{\prime}
$$


such that the new connection

$$
\left(\tilde{E}^{\prime}, \tilde{\nabla}^{\prime}\right):=\phi_{*}(\tilde{E}, \tilde{\nabla})
$$

is still logarithmic, having poles at $\omega_{i}$, with eigenvalues $\theta_{i}$ and $1-$ $\theta_{i}$, for $i=0,1, t, \infty$. This is done by applying successive elementary transformations $\mathrm{elm}^{+}$with respect to the parabolic structure $\tilde{\boldsymbol{l}}$ over each singular point (see section 8.10 for the definition and properties of $\operatorname{elm}_{l}^{+}$):

$$
\phi=\operatorname{elm}_{\tilde{l}_{0}}^{+} \circ \operatorname{elm}_{\tilde{l}_{1}}^{+} \circ \operatorname{elm}_{\tilde{l}_{t}}^{+} \circ \operatorname{elm}_{\tilde{l}_{\infty}}^{+} .
$$

The new connection is no more trace free: we have

$$
\operatorname{det}\left(\tilde{E}^{\prime}\right)=\mathcal{O}_{X}\left(\left[\omega_{0}\right]+\left[\omega_{1}\right]+\left[\omega_{t}\right]+\left[\omega_{\infty}\right]\right) \simeq \mathcal{O}_{X}\left(4\left[\omega_{\infty}\right]\right)
$$

and the $\operatorname{trace} \operatorname{tr}\left(\tilde{\nabla}^{\prime}\right)$ is the unique logarithmic connection on $\mathcal{O}_{X}\left(4\left[\omega_{\infty}\right]\right)$ having poles at each $\omega_{i}$ with residue +1 and trivial monodromy. Over the affine chart $X^{*}, \operatorname{tr}\left(\tilde{\nabla}^{\prime}\right)$ is defined in a convenient trivialization of the line bundle by

$$
d-\left(\frac{d x}{x}+\frac{d x}{x-1}+\frac{d x}{x-t}\right) .
$$

Step 3 : We now twist $(\tilde{E}, \tilde{\nabla})$ by a convenient rank one connection in order to restore the trace-free property. For this, we choose the unique square root $(L, \zeta)$ of $\left(\operatorname{det}\left(\tilde{E}^{\prime}\right), \operatorname{tr}\left(\tilde{\nabla}^{\prime}\right)\right)$ defined on the line bundle $L=\mathcal{O}_{X}\left(2\left[\omega_{\infty}\right]\right): \zeta$ is given over the affine chart by

$$
d-\left(\frac{d x}{2 x}+\frac{d x}{2(x-1)}+\frac{d x}{2(x-t)}\right) .
$$

The resulting $\operatorname{sl}(2, \mathbb{C})$-connection

$$
\left(\tilde{E}^{\prime \prime}, \tilde{\nabla}^{\prime \prime}\right):=\left(\tilde{E}^{\prime}, \tilde{\nabla}^{\prime}\right) \otimes(L, \zeta)^{\otimes(-1)}
$$

has exponent $2 \theta_{i}-1$ over $\omega_{i}, i=0,1, t, \infty$. For special parameters

$$
\boldsymbol{\theta}=\left(\frac{1}{2}, \frac{1}{2}, \frac{1}{2}, \frac{1}{2}+\frac{\vartheta}{2}\right)
$$

$\left(\tilde{E}^{\prime \prime}, \tilde{\nabla}^{\prime \prime}\right)$ is a Lamé connection (i.e. having a single pole at $\infty$ ) with exponent $\vartheta\left(\right.$ at $\left.\omega_{\infty}\right)$; as we shall see, all irreducible Lamé connections can be obtained by this way. When $\vartheta$ is an odd integer, we note that $\omega_{\infty}$ is a parabolic singular point of $\tilde{\nabla}^{\prime \prime}$ if, and only if, $\infty$ is parabolic for $\nabla$; when $\vartheta$ is even, $\omega_{\infty}$ is always apparent for the Lamé connection $\tilde{\nabla}^{\prime \prime}$ (never parabolic). 


\section{Computing the Vector Bundle of AN Elliptic PUlL-Back}

Under notations of section 2, we would like to determine the vector bundle $\tilde{E}^{\prime \prime}$ over the elliptic curve $X$ in terms of the initial connection $(E, \nabla)$. In fact, the construction of $\tilde{E}^{\prime \prime}$ only depend on $E$ and the parabolic structure $\boldsymbol{l}=\left(l_{0}, l_{1}, l_{t}, l_{\infty}\right)$. We restrict our attention to irreducible connections for simplicity, although the general case could be handled by similar arguments. The goal of this section is to prove the

Theorem 5. Let $(E, \nabla, l)$ be an irreducible parabolic connection as before and $\left(\tilde{E}^{\prime \prime}, \tilde{\nabla}^{\prime \prime}\right)$ its elliptic pull-back. Then $\tilde{E}^{\prime \prime}$ is semistable and we are in one of the following cases:

- $E$ is the trivial bundle and not three lines $l_{i}$ coincide.

In particular, the cross-ratio

$$
c=\frac{l_{t}-l_{0}}{l_{1}-l_{0}} \frac{l_{1}-l_{\infty}}{l_{t}-l_{\infty}} \in \mathbb{P}^{1}
$$

is well defined and we have:

$$
\lambda\left(\tilde{E}^{\prime \prime}\right)=t \frac{c-1}{c-t}
$$

Precisely, $\tilde{E}^{\prime \prime}$ is undecomposable if, and only if

- either $c=t$ (the diagonal case)

- or $c=0,1, \infty$ and only two lines $l_{i}$ coincide (the other two ones being mutually distinct).

- $E=\mathcal{O}(-1) \oplus \mathcal{O}(1)$ and none of the lines $l_{i}$ coincides with the sub-line-bundle $\mathcal{O}(1)$. Then $\tilde{E}^{\prime \prime}$ is the trivial bundle or the undecomposable one $E_{0}$ depending on the fact that all $l_{i}$ lie on a sub-line-bundle $\mathcal{O}(-1) \hookrightarrow E$ or not.

For the application we have in mind, one should emphasize that, along the isomonodromic deformation $\left(E_{t}, \nabla_{t}\right)$ of such a connection $(E, \nabla)$, the set of parameters $t$ for which $E_{t}$ is not trivial is always a strict (possibly empty) analytic subset of the parameter space $T$ (see [16]).

Let us start the proof of Theorem 5 by justifying restrictions on $E$ and $\boldsymbol{l}$. This will follow from

Lemma 6. Let $X$ be a curve of genus $g, E$ a vector bundle, $\nabla: E \rightarrow$ $E \otimes \Omega(D)$ a logarithmic connection with reduced effective divisor $D$, and $L \subset E$ a line bundle which is not $\nabla$-invariant. Then, the integer

$$
\nu:=\operatorname{deg}(E)+\operatorname{deg}(D)+2 g-2-2 \operatorname{deg}(L) \geq 0
$$

is bounding the number of poles of $\nabla$ where $L$ coincides with an eigenline. 
Here, we mean the eigenline of the residue of $\nabla$.

Proof. The composition

$$
L \stackrel{\text { inclusion }}{\longrightarrow} E \stackrel{\nabla}{\longrightarrow} E \otimes \Omega(D) \stackrel{\text { quotient }}{\longrightarrow} E / L \otimes \Omega(D)=: L^{\prime}
$$

defines an homomorphism of line bundles $L \rightarrow L^{\prime}$, and thus a section

$$
\phi \in \mathrm{H}^{0}\left(X, L^{\prime} \otimes L^{-1}\right)=\mathrm{H}^{0}\left(X, \operatorname{det}(E) \otimes \Omega(D) \otimes L^{-2}\right) .
$$

When $L$ is not $\nabla$-invariant, then $\phi$ is a non trivial section vanishing precisely at

- non singular points of $\nabla$ where $L$ is stabilized: $\nabla(L) \subset L \otimes$ $\Omega(D)$,

- poles of $\nabla$ where $L$ coincides with an eigenline of the residue of $\nabla$.

This gives the result.

In our situation, $g=0$ and $\operatorname{deg}(D)=4$; we deduce that 2 $2 \operatorname{deg}(L) \geq 0$ for any line bundle $L \subset E$ (since $\nabla$ is irreducible, no line bundle $L$ can be $\nabla$-invariant). Therefore, $E$ is either trivial, or $\mathcal{O}(-1) \oplus \mathcal{O}(1)$. In the former caser, any embedding $\mathcal{O} \hookrightarrow E$ passes through at most two eigenlines; in the later case, $L=\mathcal{O}(1)$ passes through no eigenlines.

3.1. Projective bundles. In order to compute the modular invariant $\lambda\left(\tilde{E}^{\prime \prime}\right)$ for the elliptic pull-back, it is more convenient to work with the associated projective bundle. If $E$ is a vector bundle over a curve $X$, then we denote by $\mathbb{P}(E)$ the associated $\mathbb{P}^{1}$-bundle. Another vector bundle $E^{\prime}$ gives rise to the same $\mathbb{P}^{1}$-bundle if, and only if, $E^{\prime}=L \otimes E$ for a line bundle $L$. When $E$ and $E^{\prime}$ are both determinant free, then $L$ is a 2-torsion point of the jacobian: there are at most $2^{2 g}$ determinant free vector bundles $E^{\prime}$ giving rise to the same $\mathbb{P}^{1}$-bundle, where $g$ is the genus of $X$. Line bundles $L \subset E$ are in one-to-one correspondence with sections $\sigma: X \rightarrow \mathbb{P}(E)$. The total space $S$ of $\mathbb{P}(E)$ fits naturally into a ruled surface and any section $\sigma$ defines a curve on $S$ that we still denote by $\sigma$ for simplicity; the normal bundle of $\sigma$ in $S$ identifies with the line bundle $\operatorname{det}(E) \otimes L^{-2}$ where $L \subset E$ is the corresponding line bundle, so that the self-intersection is given by

$$
\sigma \cdot \sigma=\operatorname{deg}(E)-2 \operatorname{deg}(L) .
$$

Recall that $E$ is semistable if, and only if, $\operatorname{deg}(E)-2 \operatorname{deg}(L) \geq 0$ for any line bundle $L \subset E$. If $L^{\prime}$ is any line bundle distinct from $L$, then the composition

$$
L^{\prime} \rightarrow E \rightarrow E / L \simeq \operatorname{det}(E) \otimes L^{-1}
$$


is a non trivial homomorphism of line bundles; this defines a non trivial holomorphic section of $\operatorname{det}(E) \otimes L^{-1} \otimes L^{\prime-1}$ vanishing at those points where $L$ and $L^{\prime}$ coincide. For the corresponding sections $\sigma$ and $\sigma^{\prime}$, we deduce the intersection number

$$
\sigma \cdot \sigma^{\prime}=\operatorname{deg}(E)-\operatorname{deg}(L)-\operatorname{deg}\left(L^{\prime}\right)=\frac{1}{2}\left(\sigma \cdot \sigma+\sigma^{\prime} \cdot \sigma^{\prime}\right) \geq 0 .
$$

A vector bundle $E$ is decomposable, i.e. of the form $E=L \oplus L^{\prime}$, if and only if $\mathbb{P}(E)$ admits two disjoint sections $\sigma$ and $\sigma^{\prime}$ (which obviously correspond to $L$ and $L^{\prime}$ respectively), or equivalently two sections $\sigma$ and $\sigma^{\prime}$ having opposite self-intersection numbers. In this case, the $\mathbb{P}^{1}$-bundle $\mathbb{P}(E)$ may be viewed as the fibre-compactification $\overline{L^{\prime} \otimes L^{-1}}$ of the line bundle $L^{\prime} \otimes L^{-1}$ obtained by adding a section at infinity. Precisely, $\sigma$ (resp. $\sigma^{\prime}$ ) stands for the null section (resp. the section at infinity) in $\mathbb{P}(E) \simeq \overline{L^{\prime} \otimes L^{-1}}$.

When $X$ is an elliptic curve, say $X:\left\{y^{2}=x(x-1)(x-t)\right\}$, and $\operatorname{det}(E)=\mathcal{O}_{X}$, then $E$ is semistable if, and only if, $\mathbb{P}(E)$ has a section having zero self-intersection. The four undecomposable bundles defined by (8) correspond to the same $\mathbb{P}^{1}$-bundle $\mathbb{P}\left(E_{0}\right)$ defined by the unique non trivial extension

$$
0 \rightarrow \mathcal{O}_{X} \rightarrow E_{0} \rightarrow \mathcal{O}_{X} \rightarrow 0 .
$$

The corresponding ruled surface $S_{0}$ is characterized by the fact that it admits one, and exactly one section having zero self-intersection. Following Atiyah [2, 39], all other semistable and determinant free vector bundles are decomposable: the corresponding $\mathbb{P}^{1}$-bundle takes the form

$$
\mathbb{P}(E)=\overline{\mathcal{O}_{X}([\omega]-[-\omega])}
$$

where $\pm \omega \in X$ are the two points of the fiber $\pi^{-1}(\lambda(E)$ ) (see definition (17)). We note that the modular invariant $\lambda(E)$ is determined by $\mathbb{P}(E)$ up to the action of the 2-torsion points of the elliptic curve $X$ : determinant free vector bundles with $\mathbb{P}^{1}$-bundle $\mathbb{P}(E)$ are the four semistable bundles with modular invariants

$$
\lambda, \quad \frac{t}{\lambda}, \frac{\lambda-t}{\lambda-1} \text { and } t \frac{\lambda-1}{\lambda-t} .
$$

3.2. Ruled surfaces and elliptic pull-back. Let us now describe the construction of section 2 in terms of ruled surfaces. We start from a rational ruled surface $p: S=\mathbb{P}(E) \rightarrow \mathbb{P}^{1}$ equipped with the parabolic structure $\boldsymbol{l}$ defined by a point $l_{i}$ on the fibre $\left.S\right|_{i}=p^{-1}(i)$ for $i=$ $0,1, t, \infty$.

step 1 : The elliptic ruled surface $\tilde{p}: \tilde{S}=\mathbb{P}(\tilde{E}) \rightarrow \mathbb{P}^{1}$ is obtained after a two-fold ramified cover $\Pi: \tilde{S} \rightarrow S$ ramifying over the four fibres $\left.S\right|_{i}$ 
and makes commutative the following diagram

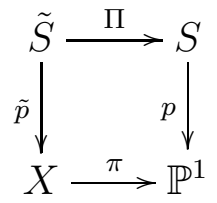

We equipp $\tilde{S}$ with the parabolic structure $\tilde{\boldsymbol{l}}$ defined by $\tilde{l}_{i}=\Pi^{-1}\left(l_{i}\right) \in$ $\left.\tilde{S}\right|_{\omega_{i}}=\tilde{p}^{-1}\left(\omega_{i}\right)$ for $i=0,1, t, \infty$.

step 2 (and 3) : The birational transformation



is obtained by blowing-up the four points $\tilde{l}_{i}$, and then blowing-down the strict transform of the four fibers. Step 3 is not relevant from the projective point of view since we just multiply $\tilde{E}^{\prime}$ by a line bundle in order to obtain $\tilde{E}^{\prime \prime}$. Therefore, $\tilde{S}^{\prime \prime}=\tilde{S}^{\prime}$.

3.3. Elliptic ruled surfaces and elementary transformations. From Lemma 6, we know that the parabolic ruled surface $(S, \boldsymbol{l})$ we start with is

- either $S=\mathbb{P}^{1} \times \mathbb{P}^{1}$ and not three points $l_{i} \in S$ lie on the same horizontal line,

- or $S=\mathbb{F}_{2}$ is the second Hirzebruch surface, the total space of $\mathbb{P}(\mathcal{O}(-1) \oplus \mathcal{O}(1))$, and no point $l_{i}$ lie on the "negative" section $\sigma$ corresponding to $\mathcal{O}(1)$.

In each case, we denote by $\tilde{S}^{\prime}$ the corresponding elliptic pull-back constructed in section 3.2 ,

Proposition 7. When $S=\mathbb{P}^{1} \times \mathbb{P}^{1}$, choose a coordinate $w$ and denote by

$$
c=\frac{w_{t}-w_{0}}{w_{1}-w_{0}} \frac{w_{1}-w_{\infty}}{w_{t}-w_{\infty}} \in \mathbb{P}^{1}
$$

the cross-ratio where $w_{i}$ are defined by $l_{i}=\left(i, w_{i}\right)$. Then we have:

- when $c \neq 0,1, t, \infty$, then $\tilde{S}^{\prime}$ is the decomposable ruled surface

$$
\tilde{S}^{\prime}=\overline{\mathcal{O}_{X}([\omega]-[-\omega])}
$$

where $\pm \omega=(x, \pm y) \in X$ are the two points over $x=t \frac{c-1}{c-t}$;

- when $c=t$ (all four points $l_{i}$ lie on the same irreducible bidegree $(1,1)$-curve) then $\tilde{S}^{\prime}$ is the undecomposable ruled surface $S_{0}$; 
- when $c=0,1, \infty$, then at least two points $l_{i}$ lie on the same horizontal line; if the two other points lie on another horizontal line, then $\tilde{S}^{\prime}$ is the trivial bundle; else, $\tilde{S}^{\prime}$ is the undecomposable ruled surface $S_{0}$.

When $S=\mathbb{F}_{2}$, then we have:

- when the four points $l_{i}$ lie on a section having +2 self-intersection (i.e. induced by any embedding $\mathcal{O}_{\mathbb{P}^{1}}(-1) \hookrightarrow \mathcal{O}_{\mathbb{P}^{1}}(-1) \oplus \mathcal{O}_{\mathbb{P}^{1}}(1)$ ) then $\tilde{S}^{\prime}$ is the trivial bundle;

- else, $\tilde{S}^{\prime}$ is the undecomposable ruled surface $S_{0}$.

Proof. We first consider the generic case $S=\mathbb{P}^{1} \times \mathbb{P}^{1}$ and $c \neq 0,1, t, \infty$. One can choose the vertical coordinate $w$ such that

$$
l_{0}=(0,0), \quad l_{1}=(1,1), \quad l_{t}=(t, c) \quad \text { and } \quad l_{\infty}=(\infty, \infty) .
$$

One easily check by computation that there is a unique curve $C \subset$ $\mathbb{P}^{1} \times \mathbb{P}^{1}$ of bidegree $(2,2)$ intersecting each fibre $x=i$ at the point $l_{i}$ with multiplicity 2 . The equation for $C$ is given by $F=0$ where

$$
F(x, w)=((c-t) x-t(c-1)) w^{2}+2(t-1) c x w-c x((c-1) x-(c-t)) .
$$

The discriminant with respect to the $w$-variable, given by

$$
\operatorname{disc}_{y}(F)=4 c(c-1)(c-t) x(x-1)(x-t),
$$

is not identically vanishing, with simple roots; we deduce that the curve $C$ is reduced, irreducible and smooth. Its lifting $\tilde{C}$ to the trivial bundle $X \times \mathbb{P}^{1}$ splits into the union of 2 distinct sections $\tilde{\sigma}_{0}$ and $\tilde{\sigma}_{\infty}$ intersecting exactly at the 4 points $\tilde{l}_{i}$ without multiplicity. After elementary transformations with center $\tilde{l}_{i}$, we obtain two disjoint sections $\sigma_{0}$ and $\sigma_{\infty}$ of $\tilde{S}^{\prime}$; we already deduce that $\tilde{S}^{\prime}$ is the compactification of a line bundle. In order to determine this line bundle, consider the horizontal section $w=\infty$ of $\mathbb{P}^{1} \times \mathbb{P}^{1}$ passing through $l_{\infty}$ : it intersects the $(2,2)$-curve $C$ in 2 points, namely $l_{\infty}$ and the point $s=(x, \infty)$ with coordinate $x=t \frac{c-1}{c-t}$; it lifts to a section $\tilde{\sigma}$ of $X \times \mathbb{P}^{1}$ intersecting $\tilde{\sigma}_{0}$ over 0 and, say, $\omega$, and intersecting $\tilde{\sigma}_{\infty}$ over 0 and $-\omega$ where $\pm \omega \in X$ are the two points of $X$ over $x$. After elementary transformations with centers $\tilde{l}_{i}$, we derive a section $\sigma$ of $\tilde{S}^{\prime}$ intersecting $\sigma_{0}$ at $\omega$ and $\sigma_{\infty}$ at $-\omega$ : we thus obtain $\tilde{S}^{\prime}=\overline{\mathcal{O}([\omega]-[-\omega])}$. This is resumed in picture 1].

When $c=t$, the curve $F=0$ degenerates to twice the $(1,1)$-curve passing through all $l_{i}$, namely the diagonal section $\sigma(x)=x$. Its lifting to $\tilde{S}$ is the graph $\tilde{\sigma}$ of the two-fold cover $X \rightarrow \mathbb{P}^{1}$ having self-intersection +4. After elementary transformations, we deduce a section $\tilde{\sigma}^{\prime}$ of $\tilde{S}^{\prime}$ having zero self-intersection. More precisely, the normal bundle of $\tilde{\sigma}^{\prime}$ is the trivial bundle. Indeed, the section $\tilde{\sigma}$ is induced in $\tilde{S}=\mathbb{P}(\tilde{E})$, 



Figure 1 . Lifting $\mathbb{P}(\nabla)$ on the elliptic curve $E_{t}$ 
$\tilde{E}=\mathcal{O}_{X} \oplus \mathcal{O}_{X}$, by the line bundle $\tilde{L} \subset \tilde{E}$ generated by its meromorphic section $(x, y) \mapsto\left(\begin{array}{l}x \\ 1\end{array}\right)$, whose divisor is $-2\left[\omega_{\infty}\right]$; the normal bundle of $\tilde{\sigma}$ in $\tilde{S}$ is therefore given by

$$
\mathcal{N}_{\tilde{\sigma}}=\operatorname{det}(\tilde{E}) \otimes \tilde{L}^{-2}=\mathcal{O}_{X} \otimes \mathcal{O}_{X}\left(-2\left[\omega_{\infty}\right]\right)^{-2}=\mathcal{O}_{X}\left(4\left[\omega_{\infty}\right]\right) .
$$

After elementary transformations, we obtain

$$
\begin{gathered}
\mathcal{N}_{\tilde{\sigma}^{\prime}}=\operatorname{det}\left(\tilde{E}^{\prime}\right) \otimes L^{\prime-2} \\
=\mathcal{O}_{X}\left(\left[\omega_{0}\right]+\left[\omega_{1}\right]+\left[\omega_{t}\right]+\left[\omega_{\infty}\right]\right) \otimes \mathcal{O}_{X}\left(\left[\omega_{0}\right]+\left[\omega_{1}\right]+\left[\omega_{t}\right]-\left[\omega_{\infty}\right]\right)^{-2} \\
=\mathcal{O}_{X} .
\end{gathered}
$$

If $\tilde{S}^{\prime}$ were decomposable, it would be the trivial $\mathbb{P}^{1}$-bundle, what we have now to exclude. Consider the centers $\tilde{l}_{i}^{\prime} \in \tilde{S}^{\prime}$ of the inverse elementary transformations. If $\tilde{S}^{\prime}$ were the trivial $\mathbb{P}^{1}$-bundle, most of horizontal sections would avoid the four points $\tilde{l}_{i}^{\prime}$ and would define, back to $\tilde{S}=X \times \mathbb{P}^{1}$, a section having -4 self-intersection, impossible. Thus $\tilde{S}^{\prime}$ is the undecomposable bundle $S_{0}$.

We now assume $c=\infty$; the other cases $c=0$ or 1 are similar. We are in one of the following three cases:

- $w_{0}=0, w_{1}=1$ and $w_{t}=w_{\infty}=\infty$;

- $w_{0}=w_{1}=0, w_{t}=1$ and $w_{\infty}=\infty$;

- $w_{0}=w_{1}=0$ and $w_{t}=w_{\infty}=\infty$.

The last case is easy since the three horizontal sections $\sigma_{0}, \sigma_{1}$ and $\sigma_{\infty}$, respectively defined by $w=0, w=1$ and $w=\infty$, are transformed in the elliptic pull-back $\tilde{S}^{\prime}$ into disjoint sections $\tilde{\sigma}_{0}^{\prime}$ and $\tilde{\sigma}_{\infty}^{\prime}$, and a third section $\tilde{\sigma}_{1}^{\prime}$ that intersects $\tilde{\sigma}_{0}^{\prime}$ at $\omega_{t}$ and $\omega_{\infty}$ and $\tilde{\sigma}_{\infty}^{\prime}$ at $\omega_{0}$ and $\omega_{1}$. We promptly deduce that

$$
\tilde{S}^{\prime}=\overline{\mathcal{O}_{X}\left(\left[\omega_{t}\right]+\left[\omega_{\infty}\right]-\left[\omega_{0}\right]-\left[\omega_{1}\right]\right)}=X \times \mathbb{P}^{1} .
$$

We now study the first case, where only $w_{t}$ and $w_{\infty}$ coincide; it is similar to the diagonal case. The section $w=\infty$ of $S$ defines a section $\tilde{\sigma}_{\infty}^{\prime}$ of $\tilde{S}^{\prime}$ having even trivial normal bundle. If $\tilde{S}^{\prime}$ were decomposable, it would be the trival bundle, and a generic constant section $\tilde{\sigma}^{\prime}$ would provide a section $\tilde{\sigma}$ of the trivial bundle $\tilde{S}$ having -4 self-intersection, contradiction. Thus $\tilde{S}^{\prime}$ is the undecomposable bundle $S_{0}$.

Finally, let us consider the case where $S=\mathbb{F}_{2}$. Like before, one easily shows that the exceptional section $\sigma_{\infty}$ induced by $\mathcal{O}_{\mathbb{P 1}}(1)$ yields a section $\tilde{\sigma}_{\infty}^{\prime}$ of $\tilde{S}^{\prime}$ having trivial normal bundle. Again, like in the diagonal case, consider the centers $l_{i}^{\prime} \in \tilde{S}^{\prime}$ of elementary transformations inversing $\phi$ : they are contained in $\tilde{\sigma}_{\infty}^{\prime}$. If $\tilde{S}^{\prime}$ is the trivial bundle, then constant sections $\tilde{\sigma}^{\prime}$ give rise to a pencil of sections $\tilde{\sigma}$ of $\tilde{S}$ having the 
four points $\tilde{l}_{i}$ as base points. A special member of the pencil is given by the union of $\tilde{\sigma}_{\infty}$ and the four fibres over $\omega_{i}$. In fact, this pencil consists in all sections of $\tilde{S}$ passing through all $\tilde{l}_{i}$ that do not intersect $\tilde{\sigma}_{\infty}$ (plus the special one). Now, the elliptic involution $\tau:(x, y) \mapsto(x,-y)$ permutes those sections, therefore acting on the parameter space $\mathbb{P}^{1}$ : there are at least 2 fixed points, namely $\tilde{\sigma}_{\infty}$ and another section $\tilde{\sigma}_{0}$ that can be pushed down as a section $\sigma_{0}$ of $S$; by construction, $\sigma_{0}$ passes through all points $l_{i}$ and does not intersect $\sigma_{\infty}$ : it's a +2 -curve as required. Conversely, when all $l_{i}$ are contained in a +2 -curve $\sigma_{0}$, we deduce a second section $\tilde{\sigma}_{0}^{\prime}$ of $\tilde{S}^{\prime}$ yielding a trivialization. We note that the pencil considered above comes from a pencil not of sections of $S$, but of curves intersecting twice a fibre.

\section{Isomonodromic Deformations And the PAinlevé VI EQUATION}

In this section, we recall how isomonodromic deformations of logarithmic $\operatorname{sl}(2, \mathbb{C})$-connections $\left(E_{t}, \nabla_{t}\right)$ over the 4-punctured sphere are parametrized by Painlevé VI solutions, and how we can use this parametrization to compute the variation of the bundle $E_{t}^{\prime \prime}$ of the corresponding elliptic pull-back. Let us first recall what an isomonodromic deformation is.

4.1. Isomonodromic deformations and flat connections. Let $X_{0}$ be a complex projective curve and $\left(E_{0}, \nabla_{0}\right)$ be the data of a rank 2 vector bundle over $X_{0}$ equipped with a (flat) logarithmic $\operatorname{sl}(2, \mathbb{C})$ connection having (reduced) effective polar divisor $D_{0}$. Given a topologically trivial analytic deformation $\left(X_{t}, D_{t}\right)$ of the punctured curve, there is a unique deformation $\left(E_{t}, \nabla_{t}\right)$ of both the vector bundle and the connection having constant monodromy data. This just follows from the Riemann-Hilbert correspondence (proposition 23 with parameter). Monodromy data consists in the monodromy representation, completed by the "parabolic structure" at apparent singular points. Equivalently, if we denote by $\boldsymbol{X}$ the total space of the deformation, $\boldsymbol{D} \subset \boldsymbol{X}$ the (smooth) divisor, then the deformation $\left(E_{t}, \nabla_{t}\right)$ is induced by the unique flat logarithmic $\operatorname{sl}(2, \mathbb{C})$-connection $(E, \nabla)$ with polar divisor $\boldsymbol{D}$ inducing $\left(E_{0}, \nabla_{0}\right)$ on the slice $X_{0}$. In this paper, we will consider the case of the 4-punctured sphere and the once-punctured torus whose deformation are parametrized by the corresponding Teichmüller spaces that are both isomorphic to the Poincaré half-plane $\mathbb{H}$. Precisely, we start with the isomonodromic deformation of a logarithmic $\operatorname{sl}(2, \mathbb{C})$-connection $\left(E_{t}, \nabla_{t}\right)$ over the Riemann sphere with poles at 0,1 , $t$ and $\infty$ where $t$ varrying in the universal cover $T \simeq \mathbb{H} \rightarrow \mathbb{P}^{1} \backslash\{0,1, \infty\}$ 
and we consider the deformation $\left(\tilde{E}_{t}, \tilde{\nabla}_{t}\right)$ of the corresponding elliptic pull-back, as constructed in section 2. As one can easily check, $\left(\tilde{E}_{t}, \tilde{\nabla}_{t}\right)$ is still an isomonodromic deformation of a logarithmic connection over the Legendre family of elliptic curves $X_{t}$ with poles contained into the ramification locus $\left\{\omega_{0}, \omega_{1}, \omega_{t}, \omega_{\infty}\right\}$ of the elliptic curve; the parameter space $T$ is now understood as the Teichmüller space of the torus. For special parameters $\boldsymbol{\theta}=\left(\frac{1}{2}, \frac{1}{2}, \frac{1}{2}, \frac{1}{2}+\frac{\vartheta}{2}\right)$, we obtain the isomonodromic deformation of a Lamé connection.

4.2. Painlevé VI equation and fuchsian equations. Although we do not really need it, it is interesting to recall how the Painlevé VI equation was originaly derived as isomonodromic equation for fuchsian projective structures on the 4-punctured sphere with one extra branch point. After normalizing the singular points as $0,1, t$ and $\infty$ by a Moebius transformation, the corresponding fuchsian 2 nd order differential equation $u_{x x}+f(x) u_{x}+g(x) u=0$ takes the form

$$
\left\{\begin{array}{l}
f(x)=\frac{1-\kappa_{0}}{x}+\frac{1-\kappa_{1}}{x-1}+\frac{1-\kappa_{t}}{x-t}-\frac{1}{x-q} \\
g(x)=\frac{-\frac{t(t-1) H}{x-t}+\frac{q(q-1) p}{x-q}+\rho\left(\kappa_{\infty}+\rho\right)}{x(x-1)}
\end{array}\right.
$$

Here, $q \notin\{0,1, t, \infty\}$ is the branch point, $\kappa_{i}$ is the local exponent at $i=0,1, t, \infty$ and $\rho$ is fixed by the relation

$$
\kappa_{0}+\kappa_{1}+\kappa_{t}+\kappa_{\infty}+2 \rho=1 .
$$

Note that parameters $p$ and $H$ are residues of $g$

$$
H=-\operatorname{Res}_{x=t} g(x) \text { and } p=\operatorname{Res}_{x=q} g(x) .
$$

The singular point $q$ has exponent 2 ; it is apparent, i.e. a branch point of the projective chart, if and only if the parameter $H$ is given by

$$
H=\frac{q(q-1)(q-t)}{t(t-1)}\left(p^{2}-\left(\frac{\kappa_{0}}{q}+\frac{\kappa_{1}}{q-1}+\frac{\kappa_{t}-1}{q-t}\right) p+\frac{\rho\left(\kappa_{\infty}+\rho\right)}{q(q-1)}\right)
$$

Under these assumptions, the local charts $\phi=\frac{u_{1}}{u_{2}}$, where $u_{1}$ and $u_{2}$ run over independant solutions of (13), defines a projective atlas on the complement of $0,1, t, q$ and $\infty$ in the Riemann sphere. At each singular point $i=0,1, t, \infty$, one of the projective charts takes the form $\phi=z^{\kappa_{i}}$ (or possibly $\phi=\frac{1}{z^{m}}+\log (z)$ when $\kappa_{i}= \pm m \in \mathbb{Z}_{>0}$ ) for a convenient local coordinate $z$ at $i$; at $q$, one of the projective charts takes the form $\phi=z^{2}$, a simple branch point. Conversely, any projective structure on the Riemann sphere having five singularities with moderate growth, one of which being a simple branch point, is conjugated by a Moebius transformation to an element of the above family. Such a projective structure is characterized by the following data

- the position $t$ and $q$ of the singular points, 
- the exponents $\boldsymbol{\kappa}=\left(\kappa_{0}, \kappa_{1}, \kappa_{t}, \kappa_{\infty}\right)$

- and the monodromy representation of a projective chart $\phi$, up to conjugacy.

A small deformation of equation (13) with moving singular points $t$ and $q$ is said isomonodromic when the projective charts have constant monodromy representation, up to conjugacy. They are characterized by the classical

Theorem 8 (Fuchs-Malmquist). A deformation of equation (13) parametrized by the position $t$ of the singular point is isomonodromic if, and only if, exponents $\kappa_{i}$ are fixed and parameters $(p(t), q(t))$ satisfy the non autonomous hamiltonian system

$$
\frac{\partial q}{\partial t}=\frac{\partial H}{\partial p} \quad \text { and } \quad \frac{\partial p}{\partial t}=-\frac{\partial H}{\partial q}
$$

The first hamiltonian equation (17) rewrites

$$
p=\frac{1}{2}\left(\frac{t(t-1)}{q(q-1)(q-t)} \frac{\partial q}{\partial t}+\frac{\theta_{0}}{q}+\frac{\theta_{1}}{q-1}+\frac{\theta_{t}-1}{q-t}\right)
$$

Now, substituting (18) in the second equation (17) yields the Painlevé VI equation (9) with parameter $\boldsymbol{\kappa}$. From the chronological point of view, the Painlevé VI equation was first derived by Fuchs; the hamiltonian form was discovered later by Malmquist.

4.3. Painlevé VI equation and fuchsian systems. Let us now recall how Painlevé VI solutions correspond to isomonodromic deformations of logarithmic sl $(2, \mathbb{C})$-connections $\left(E_{t}, \nabla_{t}\right)$ with singular points $0,1, t$ and $\infty$ over the Riemann sphere. Let $\left(E_{t}, \nabla_{t}\right)$ be such a deformation and assume moreover that it is irreducible (this does not depend on $t$, only on the monodromy). By Lemma 6 , the underlying bundle $E_{t}$ is either trivial, or $\mathcal{O}(-1) \oplus \mathcal{O}(1)$, but it turns out that $E_{t}$ will be the trivial bundle for all but a discrete subset of the parameter $T$ : there are no non trivial irreducible isomonodromic deformations of such connections on the bundle $\mathcal{O}(-1) \oplus \mathcal{O}(1)$ (see [16]). It is thus enough to consider isomonodromic deformations of $\mathrm{sl}(2, \mathbb{C})$-Fuchsian systems

$$
\frac{d Y}{d x}=\left(\frac{A_{0}}{x}+\frac{A_{1}}{x-1}+\frac{A_{t}}{x-t}\right) Y, \quad A_{i} \in \operatorname{sl}(2, \mathbb{C}) .
$$

The residual matrix of the singular point at $x=\infty$ is given by

$$
A_{0}+A_{1}+A_{t}+A_{\infty}=0 .
$$


Let $\pm \frac{\theta_{i}}{2}$ denote the eigenvalues of $A_{i}$ :

$$
A_{i}=\left(\begin{array}{cc}
a_{i} & b_{i} \\
c_{i} & -a_{i}
\end{array}\right) \quad \text { with } \quad a_{i}^{2}+b_{i} c_{i}=\frac{\theta_{i}^{2}}{4}, \quad i=0,1, t, \infty
$$

After change of variable, $Y:=M Y$ with $M \in \mathrm{SL}(2, \mathbb{C})$, we normalize

$$
A_{\infty}=\left(\begin{array}{cc}
\frac{\theta_{\infty}}{2} & 0 \\
* & -\frac{\theta_{\infty}}{2}
\end{array}\right) .
$$

We exclude the case where $A_{\infty}=0: \quad \nabla$ is assumed singular at $\infty$. Then, we have

Theorem 9. A small deformation $A_{i}=A_{i}(t)$ of the system (19) normalized by (22) is isomonodromic if, and only if, the eigenvalues $\pm \frac{\theta_{i}}{2}$ are constant and $q:=\frac{t b_{0}}{t b_{0}+(t-1) b_{1}}$ satisfies

$$
\frac{d q}{d t}=-2 a_{0} \frac{q-1}{t-1}-2 a_{1} \frac{q}{t}+\left(1-\theta_{\infty}\right) \frac{q(q-1)}{t(t-1)}
$$

and the Painlevé VI equation (9) with parameter

$$
\boldsymbol{\kappa}=\left(\kappa_{0}, \kappa_{1}, \kappa_{t}, \kappa_{\infty}\right)=\left(\theta_{0}, \theta_{1}, \theta_{t}, \theta_{\infty}-1\right) .
$$

Let us see first how to deduce Theorem (9) from Fuchs-Malmquist Theorem (8).

Proof. The vector $\left(\begin{array}{l}0 \\ 1\end{array}\right)$ is an eigenvector for the eigenvalue $-\frac{\theta_{\infty}}{2}$ at $\infty$; by irreducibility of system (19), it is not invariant and can be choosen as a cyclic vector to derive a scalar fuchsian equation. Namely, if $Y=\left(\begin{array}{l}y_{1} \\ y_{2}\end{array}\right)$ if a solution of (19), then

$$
u:=\sqrt{x^{\theta_{0}}(x-1)^{\theta_{1}}(x-t)^{\theta_{t}}} y_{1}
$$

satisfies the scalar equation (13) with exponents

$$
\left(\kappa_{0}, \kappa_{1}, \kappa_{t}, \kappa_{\infty}\right)=\left(\theta_{0}, \theta_{1}, \theta_{t}, \theta_{\infty}-1\right)
$$

and parameters

$$
q=\frac{t b_{0}}{t b_{0}+(t-1) b_{1}}
$$

and

$$
p=\frac{a_{0}+\frac{\theta_{0}}{2}}{q}+\frac{a_{1}+\frac{\theta_{1}}{2}}{q-1}+\frac{a_{t}+\frac{\theta_{t}}{2}}{q-t} .
$$


In fact, the Darboux coordinates have the following interpretation: the point $x=q$ is the unique other point at which $\left(\begin{array}{l}0 \\ 1\end{array}\right)$ is again an eigenvector of system (19) and $-p+\frac{\theta_{0}}{2 q}+\frac{\theta_{1}}{2(q-1)}+\frac{\theta_{t}}{2(q-t)}$ is the corresponding eigenvalue. From Theorem (8), we deduce that a deformation of system (19) is isomonodromic if, and only if, the auxiliary variables $p$ and $q$ defined by (25) and (26) satisfy the hamiltonian system (17) where $H(t, p, q)$ is the non autonomous hamiltonian defined by (16).

Let us now explain how to uniquely reconstruct (19) up to gauge transformation from a solution $q(t)$ of Painlevé VI equation. First introduce auxiliary variable $p$ by formula (18). This gives us a unique scalar equation (13) from which one can reconstruct a fuchsian system by standart method. The resulting system is defined (up to gauge transformation) by (19) with equations (21) and

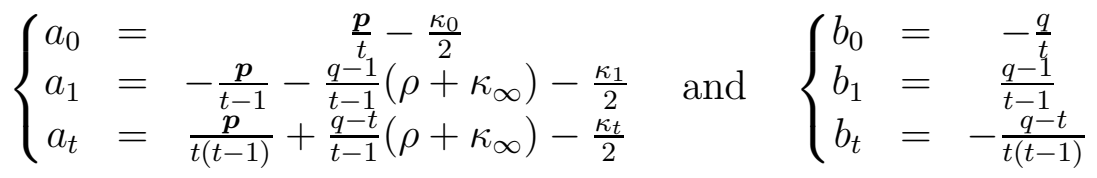

where $\boldsymbol{p}=q(q-1)(q-t) p$ and $\rho$ is defined by (14). The coefficients $c_{i}$ of the system are immediately deduced from equations (21). The standart formulae given by Jimbo and Miwa (see [4, pages 199-200) assume $\theta_{\infty} \neq 0$ so that the matrix $A_{\infty}$ can be further normalized as a diagonal matrix by additional gauge transformation; the resulting formulae look much more complicated than above ones. The way we obtain formulae (27) is described in sections 5.3, 5.4 and 5.5.

4.4. The vector bundle of an elliptic pull-back. Coming back to our initial problem, in view of applying Theorem 5, we would like parametrize the parabolic structure $\boldsymbol{l}$ induced $\nabla_{t}$ (or equivalently system (19)) by means of the Painlevé VI transcendant $q(t)$ that parametrizes the deformation. From formulae (21) and (27), we deduce that the eigenline $l_{i}$ associated to the eigenvalue $-\frac{\theta_{i}}{2}$ over the pole $i=0,1, t$ is given by

$$
l_{i}=\left(-b_{i}: a_{i}+\frac{\theta_{i}}{2}\right)
$$

which gives

$$
\left\{\begin{array}{ccc}
l_{0} & = & \left(1: \frac{\boldsymbol{p}}{q}\right) \\
l_{1} & = & \left(1: \frac{\boldsymbol{p}}{q-1}+\rho+\kappa_{\infty}\right) \\
l_{t} & = & \left(1: \frac{\boldsymbol{p}}{q-t}+\left(\rho+\kappa_{\infty}\right) t\right) \\
l_{\infty}= & (0: 1)
\end{array}\right.
$$


(recall that $l_{\infty}$ has been normalized by (22)). These expressions for $l_{i}$ are not valid anymore for a gauge equivalent system (19), for instance through Jimbo-Miwa normalization, but their cross-ratio

$$
c=\frac{l_{t}-l_{0}}{l_{1}-l_{0}} \frac{l_{1}-l_{\infty}}{l_{t}-l_{\infty}}=t \frac{(q-1) p+\rho+\kappa_{\infty}}{(q-t) p+\rho+\kappa_{\infty}}
$$

only depend on system (22) up to gauge transformation. We note that formula (29) gives an elegant definition of the auxiliary variable $p$ in terms of the parabolic structure of the connection, $q$ and $t$.

Corollary 10. Let $\left(E_{t}, \nabla_{t}\right)$ be the isomonodromic deformation defined by the Painlevé VI solution $q(t)$ like above and let $\left(\tilde{E}_{t}^{\prime \prime}, \tilde{\nabla}_{t}^{\prime \prime}\right)$ be the elliptic pull-back of the deformation. Then $\tilde{E}_{t}^{\prime \prime}$ is semistable and has invariant

$$
\lambda\left(\tilde{E}_{t}^{\prime \prime}\right)=q(t)+\frac{\rho+\kappa_{\infty}}{p(t)} .
$$

Proof. By construction, $E_{t}$ is the trivial bundle. Following Theorem 5 , $\tilde{E}_{t}^{\prime \prime}$ is semi-stable and has invariant

$$
\lambda\left(\tilde{E}_{t}^{\prime \prime}\right)=t \frac{c-1}{c-t}=q+\frac{\rho+\kappa_{\infty}}{p} .
$$

All computations above are valid only under generic assumptions that $q \neq 0,1, t, \infty$. On the other hand, it is well known that constant solutions $q(t) \equiv 0,1, t$ or $\infty$ correspond to isomonodromic deformation of reducible connections. Thus, Corollary 10 is enough to prove Theorem 1. However, we can be more precise and check at special values of $q$ and $p$ if the bundle $\tilde{E}^{\prime \prime}$ is undecomposable or not.

\section{The geometry of Painlevé VI equations}

Here, we introduce some moduli space $\mathcal{M}_{t_{0}}^{\boldsymbol{\theta}}$ of $\operatorname{sl}(2, \mathbb{C})$-connections with poles at $0,1, t_{0}$ and $\infty$ and eigenvalues $\boldsymbol{\theta}$. It will contain all irreducible connections. This space originally appeared in the work [32. of Okamoto to construct a good space of initial conditions for the Painlevé VI equation from which the Painlevé property can be read off geometrically. It is identified in [19] with a moduli space of connections, extending the dictionary established in section 4.3. After recalling this construction, we will use it to determine the vector bundle $\tilde{E}_{t}$ of an elliptic pull-back for special values of $p$ and $q$. 
5.1. Okamoto's space of initial conditions. The Painlevé property, characterizing the Painlevé equations among differential equations $\lambda^{\prime \prime}=F\left(t, \lambda, \lambda^{\prime}\right)$, says that all Painlevé VI solutions can be analytically continuated as meromorphic solutions along any path avoiding 0,1 and $\infty$. Painlevé VI solutions become meromorphic and global on the universal cover of the 3 -punctured sphere.

The (naive) space of initial conditions $\mathbb{C}^{2} \ni\left(q\left(t_{0}\right), q^{\prime}\left(t_{0}\right)\right)$ at some point $t_{0} \in \mathbb{P}^{1} \backslash\{0,1, \infty\}$ for the Painlevé VI equation fails to describe all solutions at the neighborhood of $t_{0}$. Painlevé VI solutions are meromorphic and some of them have a pole at $t_{0}$; we have to add them. The good space of initial conditions is

$$
\mathcal{M}_{t}^{\boldsymbol{\theta}}:=\left\{\text { germs of meromorphic } P_{V I^{-}}^{\boldsymbol{\theta}} \text { solutions at } t\right\}
$$

In order to construct it, Okamoto considers the phase portrait of the Painlevé VI equation in variables $\left(t, q, q^{\prime}\right) \in\left(\mathbb{P}^{1} \backslash\{0,1, \infty\}\right) \times \mathbb{C}^{2}$ (introducing auxiliary equation $\left.\frac{d q}{d t}=q^{\prime}\right)$ : it is defined by a rational vector field that defines a singular holomorphic foliation on any rational compactification. For instance, we can start with $\mathbb{P}^{1} \times \mathbb{P}^{2}$ and observe that the singularities of the foliation are located in special fibers $t=0,1, \infty$ (that we don't care) and at the infinity of the $\mathbb{P}^{2}$-factor. The latter ones are degenerate, located along a one dimensional section for the $t$-projection; we have to blow-up this section in order to reduce the degeneracy of the singular points. After 9 successive blowing-up like this, Okamoto obtains a fibre bundle

$$
t: \overline{\mathcal{M}^{\boldsymbol{\theta}}} \rightarrow \mathbb{P}^{1} \backslash\{0,1, \infty\}
$$

(we just ignore what happens over $t=0,1, \infty$ ) which is not locally trivial as an analytic bundle (it is as a topological bundle). Some non vertical divisor $Z \subset \overline{\mathcal{M}^{\boldsymbol{\theta}}}$ consists in vertical leaves (with respect to $t$-projection) and singular points. On the complement $\mathcal{M}^{\boldsymbol{\theta}}:=\overline{\mathcal{M}^{\boldsymbol{\theta}}} \backslash Z$ of this divisor, the Painlevé foliation is transversal to $t$ inducing a local analytic trivialization of the bundle

$$
t: \mathcal{M}^{\boldsymbol{\theta}} \rightarrow \mathbb{P}^{1} \backslash\{0,1, \infty\}
$$

By construction, the fibre $\mathcal{M}_{t}^{\boldsymbol{\theta}}$ at any point $t \neq 0,1, \infty$ may be interpreted as the set of germs of meromorphic $P_{V I}^{\boldsymbol{\theta}}$-solutions; actually, for special parameters $\boldsymbol{\theta}$, there are leaves staying at the infinity of the affine chart $\left(q, q^{\prime}\right)$ that cannot be viewed as meromorphic solutions, but better as "constant solutions $q \equiv \infty$ ". The divisor $Z$ actually coincides with the reduced polar divisor of the closed 2 -form defined in the affine chart by

$$
d t \wedge d H+d p \wedge d q
$$


where $H$ is defined by (16). The kernel of this 2-form defines the Painlevé foliation.

We now describe the parameter space $\mathcal{M}_{t}^{\boldsymbol{\theta}}$ starting from the Hirzebruch ruled surface $\mathbb{F}_{2}$. Define the reduced divisor $Z_{t} \subset \mathbb{F}_{2}$ as the union of the section $\sigma: \mathbb{P}^{1} \rightarrow \mathbb{F}_{2}$ having -2 self-intersection together with the 4 fibres over $0,1, t_{0}$ and $\infty$. Next, we fix 2 points on each vertical component of $Z_{t}$, none of them lying on the horizontal one. After blowing-up those 8 points, we obtain the compact space $\overline{\mathcal{M}_{t}^{\boldsymbol{\theta}}}$; still denote by $Z_{t}$ the strict transform of the divisor. The complement $\mathcal{M}_{t}^{\boldsymbol{\theta}}:=\overline{\mathcal{M}_{t}^{\boldsymbol{\theta}}} \backslash Z_{t}$ is the space of initial conditions. It remains to define the position of the 8 points in function of $\boldsymbol{\theta}$ and $t$ (see section 5.6).

5.2. Projective structures and Riccati foliations. We go back to the approach of R. Fuchs where Painlevé transcendants parametrize isomonodromic deformations of fuchsian projective structures with $4+1$ singular points (see section 4.2). Such a structure can be defined by the fuchsian 2nd order differential equation (13). One can also define it by the data of a logarithmic $\operatorname{sl}(2, \mathbb{C})$-connection $(E, \nabla)$ together with a sub line bundle $L \subset E$ which is not $\nabla$-invariant, playing the role of a cyclic vector (see section 4.3$)$. Such a data is called an $\mathrm{sl}(2, \mathbb{C}$ )-oper in [3]. A more geometrical picture inspired by the works of Ehresman is given by the projective oper defined by the triple $(\mathbb{P}(E), \mathbb{P}(\nabla), \sigma)$ where $\mathbb{P}(E)$ is the associate $\mathbb{P}^{1}$-bundle, $\mathbb{P}(\nabla)$ the induced projective connection and $\sigma: \mathbb{P}^{1} \rightarrow \mathbb{P}(E)$ the section corresponding to $L$. For instance, system (19) defines the Riccati equation

$$
\frac{d y}{d x}=-b(x) y^{2}-2 a(x) y+c(x)
$$

on the trivial $\mathbb{P}^{1}$-bundle by setting $(1: y)=\left(y_{1}: y_{2}\right)$ where $Y=$ $\left(\begin{array}{l}y_{1} \\ y_{2}\end{array}\right)$ and $A=\left(\begin{array}{cc}a & b \\ c & -a\end{array}\right)$. We preferably consider the associate phase portrait, namely the singular holomorphic foliation $\mathcal{F}_{0}$ induced on the ruled surface $\mathbb{F}_{0}=\mathbb{P}^{1} \times \mathbb{P}^{1}$, a Riccati foliation (see [6] or section 8.8). Singular points of the foliation are located at the poles of the Riccati equation. Precisely, under notations (21), the singular points are

$$
\left\{\begin{array}{l}
l_{i}=\left(-b_{i}: a_{i}+\frac{\theta_{i}}{2}\right)=\left(a_{i}-\frac{\theta_{i}}{2}: c_{i}\right) \\
l_{i}^{\prime}=\left(-b_{i}: a_{i}-\frac{\theta_{i}}{2}\right)=\left(a_{i}+\frac{\theta_{i}}{2}: c_{i}\right)
\end{array}\right.
$$

From the foliation point of view 1 , we say that $l_{i}$ and $l_{i}^{\prime}$ have respective exponents $\theta_{i}$ and $-\theta_{i}$; they correspond to the eigenlines of the system

\footnotetext{
${ }^{1}$ For instance, $1 / \theta_{i}$ is the Camacho-Sad index of $\mathcal{F}_{1}$ along the fibre $x=i$ at $l_{i}$, see [6].
} 
respectively associated to eigenvalues $-\frac{\theta_{i}}{2}$ and $\frac{\theta_{i}}{2}$ (mind the sign). In case $\theta_{i}=0$, either the singular point of the system is logarithmic and the 2 singular points of $\mathcal{F}_{1}$ coincide, or the singular point of the system is apparent and the Riccati foliation is not singular. In this latter case, we will later introduce an additional parabolic structure. The section $\sigma$ defined by $y=\infty$ plays the role of the cyclic vector; it has 2 tangencies with the Riccati foliation, namely at $x=q$ and $x=\infty$ (where $\sigma$ passes through a singular point of the foliation), see bottom of figure 2 .

In this picture, the foliation $\mathcal{F}_{0}$ is regular, transversal to the $\mathbb{P}^{1}$-fibre over a generic point $x$ and is therefore tranversely projective (see [8]). The foliation thus induces a projective structure on the section $\sigma$ that projects on the base $\mathbb{P}^{1}$. It is clear that the projective structure obtained on (a Zariski open subset of) $\mathbb{P}^{1}$ is preserved by a birational bundle transformation and it is natural to look for the simplest birational model. In the triple $\left(\mathbb{F}_{0}, \mathcal{F}_{0}, \sigma\right)$ above, the point $x=\infty$ artificially plays a special role since we impose by normalization (22) that the section $\sigma$ passes through the singular point $(x, y)=(\infty, \infty)$ of the foliation. It is more natural to apply an elementary transformation at this point and get the following more symetric picture (see [24]): a Riccati foliation $\mathcal{F}_{1}$ on the Hirzebruch ruled surface $\mathbb{F}_{1}$ having singular points over 0,1 , $t$ and $\infty$, and the section $\sigma$ has now a single tangency with the foliation at the point $x=q$. In fact, $\sigma: \mathbb{P}^{1} \rightarrow \mathbb{F}_{1}$ is the unique negative section (i.e. having -1 self-intersection). The exponents (eigenvalues) of the foliation are now given by

$$
\boldsymbol{\kappa}=\left(\kappa_{0}, \kappa_{1}, \kappa_{t}, \kappa_{\infty}\right):=\left(\theta_{0}, \theta_{1}, \theta_{t}, \theta_{\infty}-1\right) .
$$

Over each pole $x=i$ of the Riccati equation, the foliation $\mathcal{F}_{1}$ has 1 or 2 singular points depending on the exponent $\kappa_{i}$; when $q=i$, one of the singular points accidentally lie on $\sigma$, see the center of figure 2 ,

We now add a parabolic structure which is convenient for the sequel when we will apply elementary transformations; it is necessary in order to get a smooth moduli space when one of the exponents $\kappa_{i}$ vanishes.

The Okamoto space of initial conditions $\mathcal{M}_{t}^{\boldsymbol{\theta}}$ can be viewed as the moduli space of such Riccati foliations $\mathcal{F}_{1}$. Precisely, we fix exponents $\boldsymbol{\kappa}$ and parameter $t$, and then consider datas $\left(\mathbb{F}_{1}, \mathcal{F}_{1}, \sigma, \boldsymbol{l}\right)$ where

- $\mathbb{F}_{1}$ is the Hirzebruch ruled surface equipped with the ruling $\mathbb{F}_{1} \rightarrow \mathbb{P}^{1}$

- $\mathcal{F}_{1}$ is a Riccati foliation on $\mathbb{F}_{1}$ regular, transversal to the ruling outside $x=0,1, t, \infty$,

- over each $i=0,1, t, \infty$, either the fibre $x=i$ is invariant and there is one singular point for each exponent $\pm \kappa_{i}$, or $\kappa_{i}=0$ and the foliation is regular, transversal to the ruling, 
- $\boldsymbol{l}=\left(l_{0}, l_{1}, l_{t}, l_{\infty}\right)$ where either $l_{i}$ is the singular point with exponent $\kappa_{i}$ over $x=i$, or $\kappa_{i}=0, \mathcal{F}_{i}$ is regular over $x=i$ and $l_{i}$ is any point of the fibre.

Such a data exactly corresponds to the projectivization of the semistable parabolic connections considered in [19] to construct the moduli space $\mathcal{M}_{t}^{\boldsymbol{\theta}}$.

5.3. Riccati foliations on $\mathbb{F}_{2}$. After setting $y=x(x-1)(x-t) u^{\prime} / u$ in the scalar equation (13), we obtain the Riccati equation

$$
\begin{gathered}
\frac{d y}{d x}=-\frac{y^{2}}{x(x-1)(x-t)}+\left(\frac{\kappa_{0}}{x}+\frac{\kappa_{1}}{x-1}+\frac{\kappa_{t}}{x-t}+\frac{1}{x-q}\right) y \\
-\frac{q(q-1)(q-t) p}{x-q}-q(q-1) p+t(t-1) H-\rho\left(\kappa_{\infty}+\rho\right)(x-t) .
\end{gathered}
$$

Recall that $\kappa_{0}+\kappa_{1}+\kappa_{t}+\kappa_{\infty}+2 \rho=1$ and $H$ is defined by formula (16). Equation (34) defines a Riccati foliation $\mathcal{F}_{2}$ that naturally compactifies on the Hirzebruch surface $\mathbb{F}_{2}$ defined by two charts

$$
(x, y) \in\left(\mathbb{P}^{1} \backslash\{\infty\}\right) \times \mathbb{P}^{1} \text { and }(x, \tilde{y}) \in\left(\mathbb{P}^{1} \backslash\{0\}\right) \times \mathbb{P}^{1}
$$

with transition map $\tilde{y}=y / x^{2}$. The singular points of the foliation lie on the 5 fibres

$$
x=0, \quad 1, \quad t, \quad q \text { and } \infty
$$

with respective exponents (up to a sign)

$$
\kappa_{0}, \quad \kappa_{1}, \quad \kappa_{t}, \quad \kappa_{q}=1 \text { and } \kappa_{\infty} .
$$

Precisely, the singular points in the first chart $(x, y)$ are given by

$$
\begin{gathered}
\left\{\begin{array} { l } 
{ s _ { 0 } = ( 0 , 0 ) } \\
{ s _ { 0 } ^ { \prime } = ( 0 , t \kappa _ { 0 } ) }
\end{array} \quad \left\{\begin{array} { l } 
{ s _ { 1 } = ( 1 , 0 ) } \\
{ s _ { 1 } ^ { \prime } = ( 1 , ( 1 - t ) \kappa _ { 1 } ) }
\end{array} \quad \left\{\begin{array}{l}
s_{t}=(t, 0) \\
s_{t}^{\prime}=\left(t, t(t-1) \kappa_{t}\right)
\end{array}\right.\right.\right. \\
\text { and }\left\{\begin{array}{l}
s_{q}=(q, \boldsymbol{p}) \\
s_{q}^{\prime}=(q, \infty)
\end{array}\right.
\end{gathered}
$$

Here, $s_{i}$ (resp. $\left.s_{i}^{\prime}\right)$ is the singular point with exponent $\kappa_{i}\left(\right.$ resp. $\left.-\kappa_{i}\right)$ and $\boldsymbol{p}=q(q-1)(q-t) p$. At $x=\infty$, the singular points are given in chart $(x, \tilde{y})$ by

$$
\left\{\begin{array}{l}
s_{\infty}=(\infty,-\rho) \\
s_{\infty}^{\prime}=\left(\infty,-\rho-\kappa_{\infty}\right)
\end{array}\right.
$$

The "cyclic vector" is given by the section $\sigma$ defined in charts by $y=\infty$ and $\tilde{y}=\infty$ respectively. 

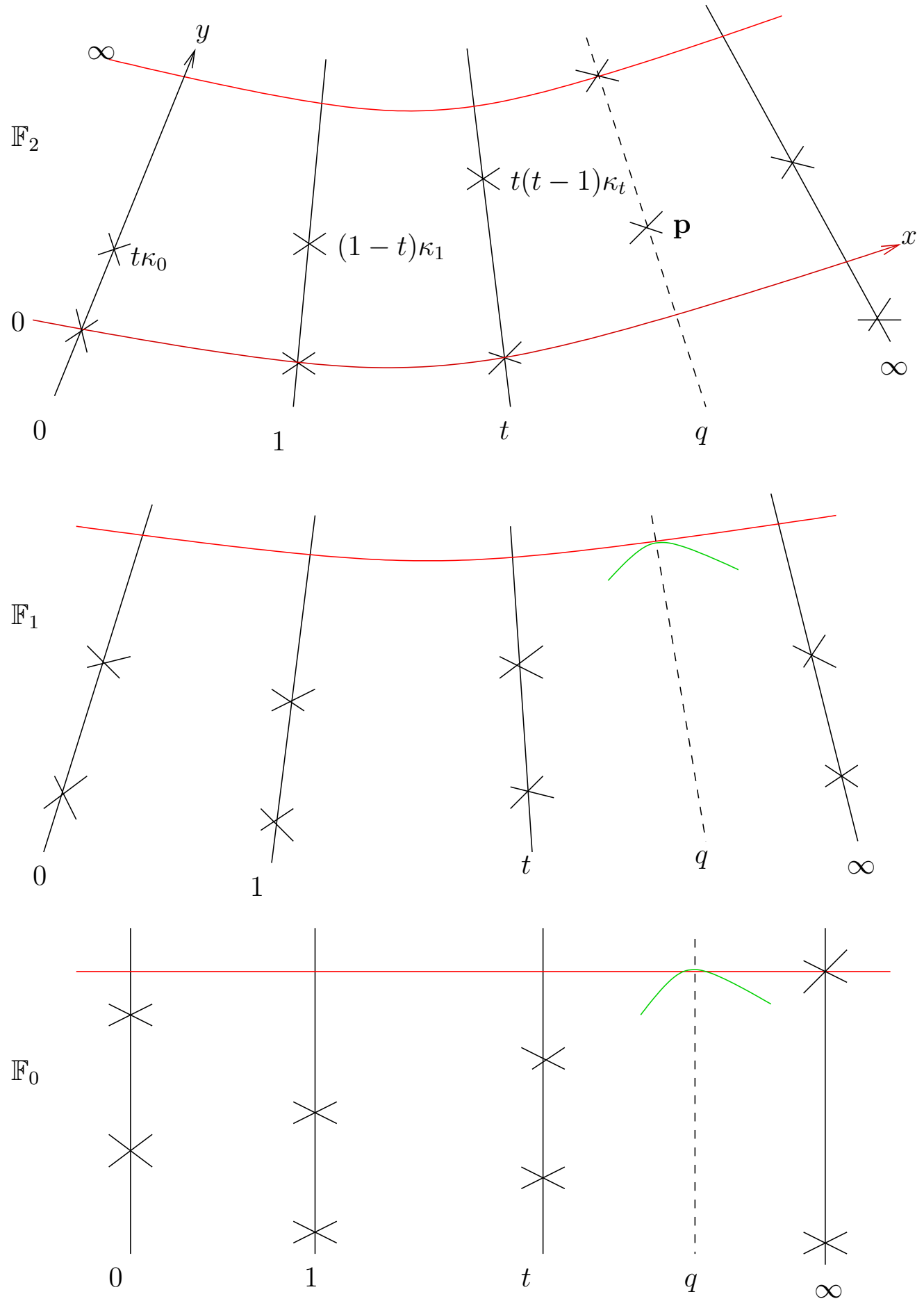

Figure 2. From $\mathbb{F}_{2}$ to $\mathbb{F}_{0}$ 
5.4. Riccati foliations on $\mathbb{F}_{1}$. After an elementary transformation with center the nodal singular point $s_{q}=(q, \boldsymbol{p})$ of $\mathcal{F}_{0}$, we obtain a Riccati foliation $\mathcal{F}_{1}$ on the Hirzebruch surface $\mathbb{F}_{1}$ with poles $x=0,1$, $t$ and $\infty$ with respective exponents $\kappa_{0}, \kappa_{1}, \kappa_{t}$ and $\kappa_{\infty}$ like in 5.2 , The apparent singular point has disappeared.

If we define $\mathbb{F}_{1}$ by usual charts

$$
(x, y) \in\left(\mathbb{P}^{1} \backslash\{\infty\}\right) \times \mathbb{P}^{1} \quad \text { and } \quad(x, \tilde{y}) \in\left(\mathbb{P}^{1} \backslash\{0\}\right) \times \mathbb{P}^{1}
$$

with transition map $\tilde{y}=y / x$, the negative section $\sigma$ is respectively given by $y=\infty$ and $\tilde{y}=\infty$, and the Riccati foliation $\mathcal{F}_{1}$ induced by

$$
\begin{gathered}
\frac{d y}{d x}=\frac{(q y-\boldsymbol{p})\left(q y-\boldsymbol{p}+t \kappa_{0}\right)}{t q x} \\
-\frac{((q-1) y-\boldsymbol{p})\left((q-1) y-\boldsymbol{p}+(1-t) \kappa_{1}\right)}{(t-1)(q-1)(x-1)} \\
+\frac{((q-t) y-\boldsymbol{p})\left((q-t) y-\boldsymbol{p}+t(t-1) \kappa_{t}\right)}{t(t-1)(q-t)(x-t)}-\rho\left(\kappa_{\infty}+\rho\right) .
\end{gathered}
$$

This equation is deduced from (37) by setting $y:=(x-q) y+\boldsymbol{p}$. The singular points in the first chart $(x, y)$ are now given by

$$
\left\{\begin{array} { l } 
{ s _ { 0 } = ( 0 , \frac { \boldsymbol { p } } { q } ) } \\
{ s _ { 0 } ^ { \prime } = ( 0 , \frac { \boldsymbol { p } - \kappa _ { 0 } } { q } ) }
\end{array} \quad \left\{\begin{array} { l } 
{ s _ { 1 } = ( 1 , \frac { \boldsymbol { p } } { q - 1 } ) } \\
{ s _ { 1 } ^ { \prime } = ( 1 , \frac { \boldsymbol { p } - ( 1 - t ) \kappa _ { 1 } } { q - 1 } ) }
\end{array} \quad \left\{\begin{array}{l}
s_{t}=\left(t, \frac{\boldsymbol{p}}{q-t}\right) \\
s_{t}^{\prime}=\left(t, \frac{\boldsymbol{p}-t(t-1) \kappa_{t}}{q-t}\right)
\end{array}\right.\right.\right.
$$

and the singular points at $x=\infty$ are given in chart $(x, \tilde{y})$ by

$$
\left\{\begin{array}{l}
s_{\infty}=(\infty,-\rho) \\
s_{\infty}^{\prime}=\left(\infty,-\rho-\kappa_{\infty}\right)
\end{array}\right.
$$

(again, $s_{i}$ has exponent $\kappa_{i}$ ).

5.5. Riccati foliations on $\mathbb{F}_{0}=\mathbb{P}^{1} \times \mathbb{P}^{1}$. Finally, after an ultimate elementary transformation with center the singular point $s_{\infty}^{\prime}$ of $\mathcal{F}_{1}$, we obtain a Riccati foliation $\mathcal{F}_{0}$ with eigenvalues

$$
\boldsymbol{\theta}=\left(\theta_{0}, \theta_{1}, \theta_{t}, \theta_{\infty}\right)=\left(\kappa_{0}, \kappa_{1}, \kappa_{t}, \kappa_{\infty}+1\right) .
$$

The underlying ruled surface depends on the relative position of the singular point $s_{\infty}^{\prime}$ of $\mathcal{F}_{1}$ with respect to the negative section $\sigma_{-1}$ : the Riccati foliation $\mathcal{F}_{0}$ is defined on

- $\mathbb{F}_{0}$ when $s_{\infty}^{\prime} \notin \sigma_{-1}$ (generic case),

- $\mathbb{F}_{2}$ when $s_{\infty}^{\prime} \in \sigma_{-1}$ (codimension 1 case). 
In particular, $q=\infty$ in the latter case.

When $q \neq \infty$, the Riccati equation defining the foliation $\mathcal{F}_{0}$ on $\mathbb{F}_{0}$ can be deduced from (37) by setting $y:=y-\left(\kappa_{\infty}+\rho\right)$. We obtain equation

$$
\begin{gathered}
-\frac{\left((q-1)\left(y-\rho-\kappa_{\infty}\right)-\boldsymbol{p}\right)\left((q-1)\left(y-\rho-\kappa_{\infty}\right)-\boldsymbol{p}+(1-t) \kappa_{1}\right)}{(t-1)(q-1)(x-1)} \\
+\frac{\left((q-t)\left(y-t\left(\kappa_{\infty}+\rho\right)\right)-\boldsymbol{p}\right)\left((q-t)\left(y-t\left(\kappa_{\infty}+\rho\right)\right)-\boldsymbol{p}+t(t-1) \kappa_{t}\right)}{t(t-1)(q-t)(x-t)} .
\end{gathered}
$$

This is precisely the projectivization of the fuchsian system defined by (27). The singular points $s_{i}$ with eigenvalues $\kappa_{i}$ correspond to the parabolic structure $\boldsymbol{l}$ defined by formula (28)

$$
\left\{\begin{array} { l } 
{ s _ { 0 } = ( 0 , \frac { \boldsymbol { p } } { q } ) } \\
{ s _ { 1 } = ( 1 , \frac { \boldsymbol { p } } { q - 1 } + \rho + \kappa _ { \infty } ) } \\
{ s _ { t } = ( t , \frac { \boldsymbol { p } } { q - t } + ( \rho + \kappa _ { \infty } ) t ) } \\
{ s _ { \infty } = ( \infty , \infty ) }
\end{array} \quad \left\{\begin{array}{l}
s_{0}^{\prime}=\left(0, \frac{\boldsymbol{p}-t \kappa_{0}}{q}\right) \\
s_{1}^{\prime}=\left(1, \frac{\boldsymbol{p}-(1-t) \kappa_{1}}{q-1}+\rho+\kappa_{\infty}\right) \\
s_{t}^{\prime}=\left(t, \frac{\boldsymbol{p}-t(t-1) \kappa_{t}}{q-t}+\left(\rho+\kappa_{\infty}\right) t\right)
\end{array}\right.\right.
$$

and

$$
\begin{gathered}
s_{\infty}^{\prime}=-\frac{\boldsymbol{p}\left(\boldsymbol{p}-t \kappa_{0}\right)}{t q\left(\kappa_{\infty}+1\right)}+\frac{\boldsymbol{p}\left(\boldsymbol{p}-(1-t) \kappa_{1}\right)}{(t-1)(q-1)\left(\kappa_{\infty}+1\right)}-\frac{\boldsymbol{p}\left(\boldsymbol{p}-t(t-1) \kappa_{t}\right)}{t(t-1)(q-t)\left(\kappa_{\infty}+1\right)} \\
(42) \quad-\frac{\kappa_{\infty}+\rho}{\kappa_{\infty}+1}\left(\left(\kappa_{\infty}+\rho\right)(q-t-1)-\kappa_{1}-t \kappa_{t}\right)
\end{gathered}
$$

5.6. The moduli space $\mathcal{M}_{t}^{\kappa}$. To each $(p, q) \in \mathbb{C} \times\left(\mathbb{P}^{1} \backslash\{0,1, t, \infty\}\right)$, we have associated a Riccati foliation $\mathcal{F}_{1}$ on the Hirzebruch surface $\mathbb{F}_{1}$ with poles $0,1, t$ and $\infty$ and exponents $\boldsymbol{\kappa}$.

Conversely, given such a Riccati foliation $\mathcal{F}_{1}$ on $\mathbb{F}_{1}$ with poles at 0 , $1, t$ and $\infty$, the unique tangency $x=q$ between the negative section $\sigma_{-1}: \mathbb{P}^{1} \rightarrow \mathbb{F}_{1}$ and the foliation $\mathcal{F}_{1}$ defines $q \in \mathbb{P}^{1}$ uniquely. Now, if $q \neq 0,1, t, \infty$, apply an elementary transformation at this tangency point to define a Riccati foliation $\mathcal{F}_{2}$ on $\mathbb{F}_{2}$. There is a unique section $\sigma_{2}: \mathbb{P}^{1} \rightarrow \mathbb{F}_{2}$ having self-intersection +2 and passing through the singular points $s_{0}, s_{1}$ and $s_{t}$ of $\mathcal{F}_{2}$; choose a chart $(x, y) \in \mathbb{C} \times \mathbb{P}^{1}$ such that the section $\sigma_{2}$ and the negative one, $\sigma_{-2}$, are respectively given by $y=0$ and $y=\infty$. In fact, $y$ is uniquely defined if we normalize the $y^{2}$-coefficient of the Riccati equation like in formula (34). Then, we observe that all singular points $s_{i}$ and $s_{i}^{\prime}$ for $i=0,1, t, \infty$ only depend on parameters $\boldsymbol{\kappa}$ and $t$; only the singular point $s_{q}$, given by $(x, y)=(q, \boldsymbol{p})$ depend on the particular foliation $\mathcal{F}_{1}$. Define $p$ 
by $\boldsymbol{p}=q(q-1)(q-t) p$. We note that the Riccati foliation $\mathcal{F}_{1}$ is characterized by the position of the nodal singular point of $\mathcal{F}_{2}$ on the Hirzebruch surface $\mathbb{F}_{2}$ (after normalization above).

Theorem ([19]). Fix parameters

$$
\boldsymbol{\kappa}=\left(\kappa_{0}, \kappa_{1}, \kappa_{t}, \kappa_{\infty}\right)
$$

and consider Riccati foliations on the Hirzebruch surface $\mathbb{F}_{1}$ having at most simple poles at $0,1, t$ and $\infty$, with exponents $\pm \kappa_{i}$ over $x=i$ such that the negative section $\sigma_{-1}$ of $\mathbb{F}_{1}$ is not $\mathcal{F}$-invariant (semi-stability condition). Add moreover a parabolic structure $\boldsymbol{l}=\left(l_{0}, l_{1}, l_{t}, l_{\infty}\right)$ which consists over each $i=0,1, t, \infty$ of the data

- either of the singular point $l_{i} \in \mathbb{F}_{1}$ with exponent $\kappa_{i}$,

- or, when $\kappa_{i}=0$ and there is actually no singular point, of any point of the fibre $x=i$ (the only case where the parabolic structure is relevant, i.e. not determined by the foliation itself).

The moduli space $\mathcal{M}_{t}^{\kappa}$ of such pairs $(\mathcal{F}, \boldsymbol{l})$ up to bundle automorphisms is a quasi-projective rational surface that can be described as follows. Start with the Hirzebruch surface $\mathbb{F}_{2}$ and denote by $Z_{t}$ the reduced divisor which consists of the 4 fibres $x=i$ together with the negative section $\sigma_{-2}$. Consider the 8 points $s_{i}$ and $s_{i}^{\prime}$ defined by

$$
\left\{\begin{array} { l } 
{ s _ { 0 } = ( 0 , 0 ) } \\
{ s _ { 0 } ^ { \prime } = ( 0 , t \kappa _ { 0 } ) }
\end{array} \quad \left\{\begin{array} { l } 
{ s _ { 1 } = ( 1 , 0 ) } \\
{ s _ { 1 } ^ { \prime } = ( 1 , ( 1 - t ) \kappa _ { 1 } ) }
\end{array} \quad \left\{\begin{array}{l}
s_{t}=(t, 0) \\
s_{t}^{\prime}=\left(t, t(t-1) \kappa_{t}\right)
\end{array}\right.\right.\right.
$$

in the first chart $(x, y)$, and

$$
\left\{\begin{array}{l}
s_{\infty}=(\infty,-\rho) \\
s_{\infty}^{\prime}=\left(\infty,-\rho-\kappa_{\infty}\right)
\end{array}\right.
$$

in the chart $\left(x, \tilde{y}=\frac{y}{x^{2}}\right)$. For $i=0,1, t, \infty$ :

- if $\kappa_{i} \neq 0$, blow-up the two points $s_{i}$ and $s_{i}^{\prime}$,

- if $\kappa_{i}=0$, blow-up the single point $s_{i}=s_{i}^{\prime}$ and then blow-up the intersection point between the exceptional divisor and the strict transform of the fibre $x=i$.

Denote by $\overline{\mathcal{M}_{t}^{\kappa}}$ the 8-point-blowing-up of $\mathbb{F}_{2}$ like above. The moduli space is $\mathcal{M}_{t}^{\kappa}$ is the complement in $\overline{\mathcal{M}_{t}^{\kappa}}$ of the strict transform of the divisor $Z_{t}$.

The 8 points $s_{i}$ and $s_{i}^{\prime}$ of the statement are nothing but the singular points of the foliation on $\mathbb{F}_{2}$ described in section 5.3. When the nodal singular point $(x, y)=(q, \boldsymbol{p})$ tends to $s_{i}$ (resp. $\left.s_{i}^{\prime}\right)$, the corresponding foliation on $\mathbb{F}_{1}$ (see section 5.4) has its singular point $s_{i}^{\prime}$ (resp. $s_{i}$ ) tending to the negative section $\sigma_{-1}$. The limit depend on the way the nodal point tends to $s_{i}$ or $s_{i}^{\prime}$. When $\kappa_{i} \neq 0$, the fibre of $\mathcal{M}_{t}^{\kappa}$ over $x=i$ 
consists in two disjoint copies $S_{i}$ and $S_{i}^{\prime}$ of the affine line $\mathbb{C}$, over $s_{i}$ and $s_{i}^{\prime}$ respectively: they stand for the moduli space of those foliations $\mathcal{F}$ whose singular point $s_{i}^{\prime}$ (resp. $s_{i}$ ) lie on the negative section $\sigma_{-1}$. When $\kappa_{i}=0$, the fibre of $\mathcal{M}_{t}^{\kappa}$ over $x=i$ consists in the union of an affine line $S_{i} \simeq \mathbb{F}$ and a projective line $S_{i}^{\prime} \simeq \mathbb{P}^{1}$ that intersect transversely at one point, and project to $s_{i}=s_{i}^{\prime}$. The component $S_{i}$ stands for those foliations for which the point $s_{i}$ lies on the negative section $\sigma_{-1}$; the compact component $S_{i}^{\prime}$ stands for those parabolic foliations $(\mathcal{F}, \boldsymbol{l})$ which have actually no pole over $x=i$. If we neglect the parabolic structure, then the rational curve $S_{i}^{\prime}$ blows down to a quadratic singular point.

Sketch of the proof of Theorem 5.6 . Let $\mathcal{F}$ be a Riccati foliation on $\mathbb{F}_{1}$ having at most simple poles over $x=0,1, t$ and $\infty$, such that the negative section $\sigma_{-1}$ is not invariant (semi-stability). In standart the chart $(x, y)$, the foliation is defined by

$$
\frac{d y}{d x}=\frac{\left(a_{1} x+a_{0}\right) y^{2}+\left(b_{2} x^{2}+b_{1} x+b_{0}\right) y+\left(c_{3} x^{3}+c_{2} x^{2}+c_{1} x+c_{0}\right)}{x(x-1)(x-t)}
$$

with $a_{k}, b_{k}, c_{k} \in \mathbb{C}, a_{0}$ and $a_{1}$ not vanishing simultaneously. Bundle automorphisms are given by changes of coordinate of the form $y:=$ $a y+b x+c, a, b, c \in \mathbb{C}, a \neq 0$. The single tangency between $\mathcal{F}$ and the negative section $\sigma_{-1}$ is given by $x=q:=-\frac{a_{0}}{a_{1}}$. Using change of coordinate $y:=a y$, one may normalize

$$
\text { either } a_{1} x+a_{0}=x-q, \quad \text { or } \quad a_{1} x+a_{0}=\tilde{q} x-1 \text {, }
$$

where $\tilde{q}=\frac{1}{q}$. Let us first assume that $q \neq \infty$ so that we may assume $a_{1} x+a_{0}=x-q$. Using a change of coordinate of the form $y:=y+b x+c$ we may further assume $b_{1}=b_{2}=0$. By the way, assuming $q \neq \infty$, we get a unique normal form

$$
\frac{d y}{d x}=\frac{(x-q) y^{2}+b_{0} y}{x(x-1)(x-t)}+\frac{c_{0}}{t x}+\frac{c_{1}}{(1-t)(x-1)}+\frac{c_{t}}{t(t-1)(x-t)}+c_{\infty}
$$

From formula (37), we get

$$
b_{0}=-2 \boldsymbol{p}+(q-1)(q-t) \kappa_{0}+q(q-t) \kappa 1+q(q-1) \kappa_{t} .
$$

The residue at $x=0$ is defined by

$$
d y=\frac{-q y^{2}+b_{0} y+c_{0}}{t} \frac{d x}{x}+(\text { holomorphic at } x=0)
$$

and the exponents $\pm \kappa_{0}$ by the discriminant

$$
\Delta_{0}:=\frac{b_{0}^{2}+4 q c_{0}}{t^{2}}=\kappa_{0}^{2} .
$$


Similarly, we get

$$
\begin{gathered}
\Delta_{1}:=\frac{b_{0}^{2}+4(q-1) c_{1}}{(t-1)^{2}}=\kappa_{1}^{2}, \quad \Delta_{t}:=\frac{b_{0}^{2}+4(q-t) c_{t}}{t^{2}(t-1)^{2}}=\kappa_{t}^{2}, \\
\text { and } \Delta_{\infty}:=1-4 c_{\infty}=\kappa_{\infty}^{2} .
\end{gathered}
$$

Once parameter $\boldsymbol{\kappa}$ is fixed, one can determine uniquely $c_{0}, c_{1}, c_{t}$ and $c_{\infty}$ in function of $\boldsymbol{\kappa}, q$ and $b_{0}$ (i.e. $\boldsymbol{p}$ ) provided that $q \neq 0,1, t, \infty$. At the neighborhood of $q=0, c_{1}, c_{t}$ and $c_{\infty}$ are still determined as functions of $\boldsymbol{\kappa}, q$ and $b_{0}$ and the moduli space of such foliations is locally isomorphic to the surface

$$
\left\{\left(q, b_{0}, c_{0}\right) \in \mathbb{C}^{3}, b_{0}^{2}+2 q c_{0}=\left(t \kappa_{0}\right)^{2}\right\} .
$$

We promptly see that the moduli space consists, over $q=0$, of an affine line parametrized by $c_{0}$ over each point $b_{0}= \pm t \kappa_{0}$.

When $\kappa_{0} \neq 0$, the graph

$$
c_{0}=-\frac{\left(b_{0}-t \kappa_{0}\right)\left(b_{0}+t \kappa_{0}\right)}{4 q}
$$

is clearly obtained by blowing-up the two points and then deleting the level $c_{0}=\infty$, i.e. the strict transform of $q=0$. From the residue (46), we deduce that are equivalent over $q=0$ :

- $q=0$ and $b_{0}=t \kappa_{0}\left(\right.$ resp. $\left.b_{0}=-t \kappa_{0}\right)$,

- $q=0$ and $\boldsymbol{p}=0$ (resp. $\boldsymbol{p}=t \kappa_{0}$ ),

- the corresponding foliation $\mathcal{F}_{1}$ has its singular point $s_{0}^{\prime}$ (resp. $s_{0}$ ) lying on the negative section $y=\infty$.

When $\kappa_{0}=0$, the graph

$$
c_{0}=-\frac{b_{0}^{2}}{4 q}
$$

is now obtained after two blowing-up and the affine line parametrized by $c_{0}$ stands for those foliations $\mathcal{F}_{1}$ whose singular point $s_{0}=s_{0}^{\prime}$ lies on the negative section $y=\infty$. The surface equation $b_{0}^{2}+4 q c_{0}=0$ has a quadratic singular point at $\left(q, b_{0}, c_{0}\right)=(0,0,0)$ that corresponds to the case where the residue (46) vanishes, i.e. $\mathcal{F}_{1}$ has actually no singular point at $x=0$. The parabolic data at $x=0$ provides the desingularization of the surface. Indeed, the moduli of pairs $\left(\mathcal{F}_{1}, \boldsymbol{l}\right)$ is locally parametrized, at the neighborhood of $q=0$, by

$$
\left\{\left(q, b_{0}, c_{0}, s_{0}\right) \in \mathbb{C}^{3} \times \mathbb{P}^{1}, b_{0}^{2}+2 q c_{0}=0,2 q s_{0}=b_{0}\right\}
$$

(or we should better write $2 q u_{0}=b_{0} v_{0}$ where $\left.\left(u_{0}: v_{0}\right)=s_{0}\right)$. The parabolic data $s_{0}=\frac{b_{0}}{2 q}$ parametrizes the exceptional divisor $S_{0}^{\prime}$ (thus providing the blowing up). The intersection $S_{0} \cap S_{0}^{\prime}$ between the two 
components, given by $\left(q, b_{0}, c_{0}, s_{0}\right)=(0,0,0, \infty)$, is the foliation without singular point at $x=0$ whose parabolic structure lies on the negative section $y=\infty$.

The study of $q=1, t, \infty$ is similar except that for $q=\infty$, we have to choose the alternate normalization

$$
\frac{d y}{d x}=\frac{(\tilde{q} x-1) y^{2}+\tilde{b}_{2} x^{2} y}{x(x-1)(x-t)}+\frac{\tilde{c}_{0}}{x}+\frac{\tilde{c}_{1}}{x-1}+\frac{\tilde{c}_{t}}{x-t}+\tilde{c}_{\infty}
$$

where $\left(\tilde{q}, \tilde{b}_{2}\right)=\left(\frac{1}{q}, \frac{b_{0}}{q^{2}}\right)$ is the other chart of $\mathbb{F}_{2}$.

The deformation $t \mapsto \mathcal{M}_{t}^{\kappa}$ is analytically trivial (but not algebraically), and the trivialization is given by the Painlevé flow (see [38, 19]). The good phase space of the Painlevé VI equation is the (locally analytically trivial) fibration

$$
t: \mathcal{M}^{\kappa} \rightarrow \mathbb{P}^{1} \backslash\{0,1, \infty\} .
$$

The map $q: \mathcal{M}_{t}^{\kappa} \rightarrow \mathbb{P}^{1}$ is regular (whenever no $\kappa_{i}=0$ ) and gives $\mathcal{M}_{t}^{\kappa}$ (for $t$ fixed) a structure of affine $\mathbb{A}^{1}$-bundle with double fibers over $0,1, t, \infty$. Finally, we note that formula (37) defines an explicit section (universal Riccati foliation)

$$
(t, q, \boldsymbol{p}) \mapsto\left(\mathbb{F}_{1}, \mathcal{F}_{1}\right)
$$

over $\mathcal{M}^{\kappa} \backslash\{q=0,1, t, \infty\}$.

5.7. The moduli space $\mathcal{M}_{t}^{\boldsymbol{\theta}}$ of $\mathrm{sl}(2, \mathbb{C})$-connections. From section 5.5. we get an isomorphism

$$
\operatorname{elm}_{s_{\infty}^{\prime}}: \mathcal{M}_{t}^{\kappa} \stackrel{\sim}{\longrightarrow} \mathcal{M}_{t}^{\boldsymbol{\theta}}
$$

from the previous moduli space, to the moduli space $\mathcal{M}_{t}^{\boldsymbol{\theta}}$ of $\mathrm{sl}(2, \mathbb{C})$ connections $(E, \nabla)$ having exponent

$$
\boldsymbol{\theta}=\left(\theta_{0}, \theta_{1}, \theta_{t}, \theta_{\infty}\right):=\left(\kappa_{0}, \kappa_{1}, \kappa_{t}, \kappa_{\infty}+1\right) .
$$

Along this moduli space, recall that the underlying vector bundle $E$ is either trivial, or $\mathcal{O}(-1) \oplus \mathcal{O}(1)$; it is actually trivial on a Zariski open set of $\mathcal{M}^{\boldsymbol{\theta}}$. The locus of the non trivial bundle is Malgrange's Theta Divisor $\Theta \subset \mathcal{M}^{\boldsymbol{\theta}}$ defined for fixed $t$ by the exceptional divisor $S_{\infty}$ obtained after blowing-up $s_{\infty}$. Indeed, this corresponds to those foliations $\mathcal{F}_{1}$ for which $s_{\infty}^{\prime}$ lie in the negative section $\sigma_{-1}$; applying $\operatorname{elm}_{s_{\infty}^{\prime}}$ gives a foliation $\mathcal{F}_{0}$ on the Hirzebruch surface $\mathbb{F}_{2}=\mathbb{P}(\mathcal{O}(-1) \oplus \mathcal{O}(1))$. In fact, we get $\mathcal{F}_{0}=\mathcal{F}_{2}$ in this case: we are back to the foliation of section 5.3 where $s_{q} \rightarrow s_{\infty}$. Precisely, if we set

$$
p=\frac{\lambda-\rho q}{(q-1)(q-t)} \quad \text { and let } \quad q \rightarrow \infty
$$


in formula (13), we then get Heun equation

$$
g(x)=\frac{\rho\left(\rho+\kappa_{\infty}+1\right) x-\lambda \kappa_{\infty}-\rho\left((t+1) \rho+\kappa_{1}+t \kappa_{t}\right)}{x(x-1)(x-t)} .
$$

For our special parameters $\boldsymbol{\theta}=\left(\frac{1}{2}, \frac{1}{2}, \frac{1}{2}, \frac{1}{2}+\frac{\vartheta}{2}\right)$, we get Lamé equation (11) with $n=\frac{\vartheta}{2}$ and $c=2 \lambda(\vartheta-1)+(t+1) \frac{\vartheta}{2}\left(\frac{\vartheta}{2}-1\right)$.

Finally, the open set $\mathcal{M}^{\boldsymbol{\theta}}-\Theta$ (resp. the closed subset $\Theta$ ) may be viewed as the moduli space of Riccati foliations $\mathcal{F}_{0}$ on $\mathbb{F}_{0}$ (resp. $\mathbb{F}_{2}$ ) having simple poles $(0,1, t, \infty)$ and exponents $\left(\theta_{0}, \theta_{1}, \theta_{t}, \theta_{\infty}\right)$ excluding those for which the section passing through $s_{\infty}$ (resp. the exceptional section) is totally $\mathcal{F}_{0}$ invariant.

5.8. Okamoto symetries. There are many birational transformations

$$
(\boldsymbol{\kappa}, t, p, q) \mapsto(\tilde{\boldsymbol{\kappa}}, \tilde{t}, \tilde{p}, \tilde{q})
$$

that induce biregular diffeomorphisms between moduli spaces

$$
\mathcal{M}^{\kappa} \rightarrow \mathcal{M}^{\tilde{\kappa}}
$$

equivariant with the projection $t$. They are studied in [33]: some of them are classical, known as Schlesinger transformations, arising from geometrical transformations on connections (resp. Riccati foliations); together with a strange extra symetry, they generate the full group of Okamoto symetries.

5.8.1. Change of signs. First of all, we can change the "spin structure", i.e. the signs of parameters $\pm \kappa_{i}$. This does not change neither the Riccati foliation, nor the coefficients $t, q, b_{0}$ of the normal form (45), but the variable $p$ is modified as follows:

$$
\begin{aligned}
(-,+,+,+): & \left\{\begin{array}{ccc}
\left(\kappa_{0}, \kappa_{1}, \kappa_{t}, \kappa_{\infty}\right) & \mapsto & \left(-\kappa_{0}, \kappa_{1}, \kappa_{t}, \kappa_{\infty}\right) \\
t & \mapsto & t \\
(q, p) & \mapsto & \left(q, p-\frac{\kappa_{0}}{q}\right)
\end{array}\right. \\
(+,-,+,+): & \left\{\begin{array}{ccc}
\left(\kappa_{0}, \kappa_{1}, \kappa_{t}, \kappa_{\infty}\right) & \mapsto & \left(\kappa_{0},-\kappa_{1}, \kappa_{t}, \kappa_{\infty}\right) \\
t & \mapsto & t \\
(q, p) & \mapsto & \left(q, p-\frac{\kappa_{1}}{q-1}\right)
\end{array}\right. \\
(+,+,-,+):\left\{\begin{array}{ccc}
\left(\kappa_{0}, \kappa_{1}, \kappa_{t}, \kappa_{\infty}\right) & \mapsto & \left(\kappa_{0}, \kappa_{1},-\kappa_{t}, \kappa_{\infty}\right) \\
t & \mapsto & t \\
(q, p) & \mapsto & \left(q, p-\frac{\kappa_{t}}{q-t}\right)
\end{array}\right) & \left\{\begin{array}{ccc}
\left(\kappa_{0}, \kappa_{1}, \kappa_{t}, \kappa_{\infty}\right) & \mapsto & \left(\kappa_{0}, \kappa_{1}, \kappa_{t},-\kappa_{\infty}\right) \\
t & \mapsto & t \\
(q, p) & \mapsto & (q, p)
\end{array}\right.
\end{aligned}
$$


5.8.2. Permutation of poles. We can now permute the 4 poles of the Riccati foliation. The order 24 group is generated by

$$
\begin{aligned}
(01):\left\{\begin{array}{clc}
\left(\kappa_{0}, \kappa_{1}, \kappa_{t}, \kappa_{\infty}\right) & \mapsto & \left(\kappa_{1}, \kappa_{0}, \kappa_{t}, \kappa_{\infty}\right) \\
t & \mapsto & 1-t \\
(q, p) & \mapsto & (1-q,-p)
\end{array}\right. \\
(1 t):\left\{\begin{array}{clc}
\left(\kappa_{0}, \kappa_{1}, \kappa_{t}, \kappa_{\infty}\right) & \left.\mapsto \kappa_{0}, \kappa_{t}, \kappa_{1}, \kappa_{\infty}\right) \\
t & \mapsto & \frac{1}{t} \\
(q, p) & \mapsto & \left(\frac{q}{t}, t p\right)
\end{array}\right. \\
(0 \infty)(1 t):\left\{\begin{array}{ccc}
\left(\kappa_{0}, \kappa_{1}, \kappa_{t}, \kappa_{\infty}\right) & \left.\mapsto \kappa_{\infty}, \kappa_{t}, \kappa_{1}, \kappa_{0}\right) \\
t & \mapsto & t \\
(q, p) & \mapsto & \left(\frac{t}{q},-\frac{q(q p+\rho)}{t}\right)
\end{array}\right.
\end{aligned}
$$

(the change of variable is given by $\tilde{x}=1-x, \frac{x}{t}$ and $\frac{t}{x}$ respectively; we get $\tilde{b}_{0}=b_{0}, \frac{b_{0}}{t^{2}}$ and $\frac{t(q-1)(q-t)-t b_{0}}{q^{2}}$ respectively).

Together with sign changes, we already get a linear group of order 384 acting on our moduli space.

5.8.3. Elementary transformations. Let $\mathcal{F}$ be a Riccati foliation on $\mathbb{F}_{1}$ representing a point of $\mathcal{M}_{t}^{\kappa}$. We can apply an elementary transformation with center one of the singular points of $\mathcal{F}$, say $s_{0}$, to obtain a new Riccati foliation with simple poles over $0,1, t$ and $\infty$ and shifted parameter $\tilde{\boldsymbol{\kappa}}=\left(\kappa_{0}-1, \kappa_{1}, \kappa_{t}, \kappa_{\infty}\right)$. The resulting bundle is either the trivial bundle $\mathbb{F}_{0}$, or the Hirzebruch surface $\mathbb{F}_{2}$. However, after two (or more generally, an even number of) such elementary transformations, we are back to $\mathbb{F}_{1}$. Indeed, since the type $n$ of the Hirzebruch surface $\mathbb{F}_{n}$ shifts by \pm 1 at each elementary transformation, we just have to exclude the possibility, say

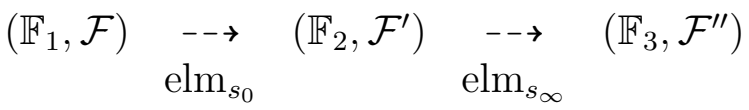

this would mean that each of the two successive elementary transformations have center lying on the negative section. In this later case, the negative section $\sigma_{-3}$ of $\mathbb{F}_{3}$ is the strict transform of $\sigma_{-1}$ : since $\sigma_{-1}$ is not $\mathcal{F}$-invariant, we get that $\sigma_{-3}$ is not $\mathcal{F}^{\prime \prime}$-invariant, but Proposition 25 gives a negative tangency, a contradiction. We have thus defined a biregular transformation

$$
\operatorname{elm}_{s_{\infty}} \circ \operatorname{elm}_{s_{0}}:\left\{\begin{array}{ccc}
\left(\kappa_{0}, \kappa_{1}, \kappa_{t}, \kappa_{\infty}\right) & \mapsto & \left(\kappa_{0}-1, \kappa_{1}, \kappa_{t}, \kappa_{\infty}-1\right) \\
t & \mapsto & t \\
(q, p) & \mapsto & (\tilde{q}, \tilde{p})
\end{array}\right.
$$


We omit the huge formula but we note that $\tilde{q}$ is given by the unique tangency point between $\mathcal{F}$ and the unique section $\sigma$ of $\mathbb{F}_{1}$ having +1 self-intersection and passing through $s_{0}$ and $s_{\infty}$.

More generally, given any 4-uple

$$
\boldsymbol{n}=\left(n_{0}, n_{1}, n_{t}, n_{\infty}\right) \in \mathbb{Z}^{4}, \quad n=n_{0}+n_{1}+n_{t}+n_{\infty} \in 2 \mathbb{Z},
$$

we construct a biregular transformation

$$
\operatorname{elm}_{l}^{n}:\left\{\begin{array}{ccc}
\left(\kappa_{0}, \kappa_{1}, \kappa_{t}, \kappa_{\infty}\right) & \mapsto & \left(\kappa_{0}-n_{0}, \kappa_{1}-n_{1}, \kappa_{t}-n_{t}, \kappa_{\infty}-n_{\infty}\right) \\
t & \mapsto & t \\
(q, p) & \mapsto & (\tilde{q}, \tilde{p})
\end{array}\right.
$$

where

$$
\operatorname{elm}_{l}^{n}=\operatorname{elm}_{s_{0}}^{n_{0}} \circ \operatorname{elm}_{s_{1}}^{n_{1}} \circ \operatorname{elm}_{s_{t}}^{n_{t}} \circ \operatorname{elm}_{s_{\infty}}^{n_{\infty}}
$$

with convention $\operatorname{elm}_{s_{i}}^{n_{i}}:=\operatorname{elm}_{s_{i}^{\prime}}^{-n_{i}}$ when $n_{i}<0$. Like before, there is a unique section $\sigma$ having self-intersection $\sigma \cdot \sigma=n-1$ and tangency of multiplicity $n_{i}$ with the foliation at each point $s_{i}$; the extra tangency between $\mathcal{F}$ and $\sigma$ is at $x=\tilde{q}$.

We now get an infinite affine group of transformations that we denote by $H$.

5.8.4. The Okamoto symetry. An extra symetry is needed to generate the full group $G$ of biregular transformations decribed in [33], namely

$$
\left\{\begin{array}{ccc}
\left(\kappa_{0}, \kappa_{1}, \kappa_{t}, \kappa_{\infty}\right) & \mapsto & \left(\kappa_{0}+\rho, \kappa_{1}+\rho, \kappa_{t}+\rho, \kappa_{\infty}+\rho\right) \\
t & \mapsto & t \\
(q, p) & \mapsto &
\end{array}\right.
$$

(called $s_{2}$ in [30]) or any of its conjugate. So far, there is no geometric interpretation of this symetry as long as we interpret $\mathcal{M}^{\kappa}$ as the moduli space of rank 2 connections (or Riccati foliations). One has to deal with isomonodromic deformations of connections of rank 3 (see [4]) or more (see [30]) in order to derive the full Okamoto group by natural transformations on the connection.

The conjugate of the Okamoto symetry above by the sign change $(+,+,+,-)$, denoted $s_{2} s_{1} s_{2}$ in notations of [30], is given by:

$$
\left\{\begin{array}{cccc}
\left(\kappa_{0}, \kappa_{1}, \kappa_{t}, \kappa_{\infty}\right) & \mapsto & \left(\kappa_{0}+\rho+\kappa_{\infty}, \kappa_{1}+\rho+\kappa_{\infty}, \kappa_{t}+\rho+\kappa_{\infty},-\rho\right) \\
t & \mapsto & t \\
(q, p) & \mapsto & & \left(q+\frac{\rho+\kappa_{\infty}}{p}, p\right)
\end{array}\right.
$$

We recognize in $\tilde{q}=q+\frac{\rho+\kappa_{\infty}}{p}$ the Tu invariant of the underlying bundle of the elliptic pull-back (see Corollary 10). 
5.9. Special configurations. For special values of the parameter $\boldsymbol{\kappa}$, the moduli space $\mathcal{M}_{t}^{\kappa}$ has rational complete curves (independently on $t$ ). They arise from curves on $\mathbb{F}_{2}$ avoiding the negative section and passing through $s_{i}$ or $s_{i}^{\prime}$ over each value $q=0,1, t, \infty$. They correspond to the locus either of reducible connections, or connections with a apparent singular point. It turns out that there are no other complete curve in $\mathcal{M}_{t}^{\kappa}$ (see [19]), in particular, no curve for generic values of $\boldsymbol{\kappa}$. Here follow some examples.

When say $\kappa_{t}=1$ and the foliation $\mathcal{F}$ has an apparent singular point at $x=t$, then the pole disappear after one elementary transformation with center $s_{t}$. We thus obtain a Hypergeometric type Riccati foliation $\mathcal{F}_{0}$ on $\mathbb{F}_{0}$ (it cannot be $\mathbb{F}_{1}$ by Proposition 25 ), that is to say with poles at 0,1 and $\infty$, and exponents $\left(\kappa_{0}, \kappa_{1}, \kappa_{\infty}\right)$. Conversely, given $\mathcal{F}_{0}$, we reconstruct a foliation $\mathbb{F}$ like above by applying an elementary tranformation at any point of the fibre $x=t$ : this gives us a rational family of foliations $\mathcal{F}$ (parametrized by the fibre $x=t$ ). The corresponding rational curve $C$ in the moduli space $\mathcal{M}_{t}^{\kappa}$ is given by equation

$$
q(q-1) p^{2}-\left((q-1) \kappa_{0}+q \kappa 1\right) p+\frac{\left(\kappa_{0}+\kappa_{1}\right)^{2}-\kappa_{\infty}^{2}}{4} .
$$

This is the unique curve $C$ in $\mathbb{F}_{2}$ satisfying:

- $q: C \rightarrow \mathbb{P}^{1}$ has degree 2 ,

- $C$ does not intersect the negative section $\sigma_{-2}$,

- $C$ intersects the fibre $q=i$ at both $s_{i}$ and $s_{i}^{\prime}$ for $i=0,1, \infty$,

- $C$ intersects twice the fibre $q=t$ at $s_{t}$,

- $C$ is singular at $s_{t}$ : it has two smooth branches.

Proof. To compute this family in the moduli space, we start from the Riccati foliation $\mathcal{F}_{0}$ that can be normalized to

$$
\frac{d y}{d x}=\frac{-y^{2}-\left(\kappa_{0}+x \kappa_{\infty}\right) y+c x}{x(x-1)}, \quad c=\frac{\kappa_{1}^{2}-\left(\kappa_{0}+\kappa_{\infty}\right)^{2}}{4}
$$

(we exclude some reducible cases here) and choose a parabolic structure $s_{t}=(t, \lambda)$ over $x=t$. The horizontal section $y=\lambda$ is sent by the elementary transformation $\operatorname{elm}_{s t}: \mathbb{F}_{0} \rightarrow \mathbb{F}_{1}$ to the negative section: the corresponding value $q=\frac{\lambda^{2}+\kappa_{0} \lambda}{c-\kappa_{\infty} \lambda}$ corresponds to the unique tangency point between $\mathcal{F}_{0}$ and $y=\infty$; we already note that the map $\lambda \mapsto q(\lambda)$ has degree 2. Now, we observe that the foliation $\mathcal{F}=\operatorname{elm}_{s_{t}} \mathcal{F}_{0}$ defines a point over $s_{0}$ (resp. $s_{0}^{\prime}$ ) in the moduli space $\mathcal{M}_{t}^{\kappa}$ if, and only if, the parabolic structure $s_{t}=(t, \lambda)$ and and the singular point $s_{0}^{\prime}$ (resp. $s_{0}$ ) of $\mathcal{F}_{0}$ lie on the same horizontal section, namely $y=\lambda$. Indeed, the later section is transformed by $\operatorname{elm}_{s_{t}}$ into the negative section of $\mathbb{F}_{1}$. Thus the curve $C$ passes once through each point $s_{0}$ and $s_{0}^{\prime}$. The 
same holds over $q=1$ and $\infty$. Finally, when the singular point $s_{t}$ is apparent for $\mathcal{F}_{1}$, it cannot lie on the negative section $\sigma_{-1}$; overwise, after applying $\lim _{s_{t}}$, we would obtain a hypergeometric foliation on $\mathbb{F}_{2}$ having, by Proposition 25, -1 tangencies with the negative section, impossible. Now we can compute the equation of the curve. Since $C$ has degree 2 and does not intersect the negative section of $\mathbb{F}_{2}$, its equation takes the form $P^{2}+A(q) P+B(q)$ where polynomials $A$ and $B$ have respective degree 2 and 4 . The fact that $C$ passes through all points $s_{i}$ and $s_{i}^{\prime}$ but $s_{t}^{\prime}$ does not define $C$ but a pencil of curves; they are smooth and vertical at $s_{t}$ (and thus escape from $\mathcal{M}_{t}^{\kappa}$ over this point) except one of them which is singular with normal crossing at $s_{t}$.

When $\kappa_{t}=2$, foliations $\mathcal{F}$ with apparent singular points at $x=t$ are obtained as follows. Take the hypergeometric foliation $\mathcal{F}_{0}$ on $\mathbb{F}_{1}$ with exponents $\left(\kappa_{0}, \kappa_{1}, \kappa_{\infty}\right)$, choose a parabolic structure $s_{t}$ at $x=0$ and apply twice $\operatorname{elm}_{s_{t}}$. Again, we get a rational curve in the moduli space which projects down to a curve $C \subset \mathbb{F}_{2}$ satisfying:

- $q: C \rightarrow \mathbb{P}^{1}$ has degree 4 ,

- $C$ does not intersect the negative section $\sigma_{-2}$,

- $C$ intersects the fibre $q=i$ at both $s_{i}$ and $s_{i}^{\prime}$ for $i=0,1, \infty$,

- $C$ intersects the fibre $q=t$ three times at $s_{t}$, one time at $s_{t}^{\prime}$.

5.10. Bolibrukh-Heu transversality. A remarkable result of Bolibrukh [5, page 37] asserts, in our context, that the isomonodromic deformation $t \mapsto\left(E_{t}, \nabla_{t}\right) \in \mathcal{M}_{t}^{\boldsymbol{\theta}}$ of an irreducible $\mathrm{sl}(2, \mathbb{C})$-connection is "mostly" defined on the trivial bundle:

Theorem 11 (Bolibrukh). Let $t \mapsto\left(E_{t}, \nabla_{t}\right) \in \mathcal{M}_{t}^{\theta}$ be a local isomonodromic deformation. Then we are in one of the following cases:

- outside a discrete set of the parameter space $T$, the underlying bundle $E_{t}$ is trivial,

- $E_{t} \equiv \mathcal{O}(-1) \oplus \mathcal{O}(1)$ and the destabilizing subsheaf $\mathcal{O}(1)$ is $\nabla_{t^{-}}$ invariant.

In particular, when $\left(E_{t}, \nabla_{t}\right)$ is irreducible, we are in the former case. Bolibrukh proved something more general for certain logarithmic connections of arbitrary rank on the Riemann sphere. The rank 2 case has been extended in full generality, to regular/irregular $\operatorname{sl}(2, \mathbb{C})$ connections, on arbitrary Riemann surfaces, by V. Heu in [16]. We will use the following Corollary

Proposition 12. Let $t \mapsto\left(E_{t}, \nabla_{t}\right) \in \mathcal{M}_{t}^{\boldsymbol{\theta}}$ be a local isomonodromic deformation of $a \operatorname{sl}(2, \mathbb{C})$-connection. Assume that $E_{t}$ is trivial and two eigenlines $l_{i}$ and $l_{j}$ coincide along the deformation, $i, j \in\{0,1, t, \infty\}$. 
Then $\left(E_{t}, \nabla_{t}\right)$ is reducible: the constant line bundle $L_{t} \subset E_{t}$ defined by $l_{i}=l_{j}$ is $\nabla_{t}$-invariant.

Proof. After applying $\operatorname{elm}_{s_{i}}$ o $\operatorname{elm}_{s_{j}}$ to the deformation, we get an isomonodromic deformation on the Hirzebruch surface $\mathbb{F}_{2}$ which is possible only when the connection is reducible by Bolibrukh Theorem 11.

Remark 13. We have the following more general result. Consider an irreducible Riccati foliation $\mathcal{F}_{0}$ in $\mathcal{M}_{t}^{\boldsymbol{\theta}}$ defined on the trivial bundle $\mathbb{P}^{1} \times \mathbb{P}^{1}$. Sections $\sigma_{d}$ having self-intersection $d \geq 0$ ( $d$ even) form $a(d+1)$-dimensional family. The smooth curve $\sigma_{d}$ has exactly $d+2$ tangencies with $\mathcal{F}_{0}$ counted with multiplicities. Then the tangency locus can be totally contained in fibers over $\{0,1, t, \infty\}$ only at isolated points of the parameter space $T$. For $d=0$ we recover the proposition. For $d=2$ we get for instance all 4 parabolics cannot lie along a bidegree $(1,1)$ curve along the deformation.

\section{LAMÉ CONNECTIONS}

The aim of this section is to roughly describe the moduli space of Lamé connections up to biregular bundle transformations by means of the Riemann-Hilbert correspondence. Here, we fix the elliptic curve

$$
X:\left\{y^{2}=x(x-1)(x-t)\right\} .
$$

We will point out which Lamé connections are invariant under the elliptic involution

$$
\sigma: X \rightarrow X ;(x, y) \mapsto(x,-y) .
$$

In the next section, we will see that $\sigma$-invariant Lamé connections can be pushed down, via the double cover

$$
\pi: X \rightarrow \mathbb{P}^{1} ;(x, y) \mapsto x
$$

as a logarithmic connection with poles at the 4 ramification points $i=0,1, t, \infty$.

Let $(E, \nabla)$ be a Lamé connection over the elliptic curve $X$, thus having a simple pole at $\omega_{\infty}$. When the exponent $\vartheta$ is not an integer, the connection may be reduced to the following matrix form

$$
\nabla: W \mapsto d W-\Omega W, \quad \Omega=\left(\begin{array}{cc}
\frac{\vartheta}{2} \frac{d z}{z} & 0 \\
0 & -\frac{\vartheta}{2} \frac{d z}{z}
\end{array}\right)
$$

where $z \in(\mathbb{C}, 0)$ is any local coordinate of $X$ at $\omega_{\infty}$, and $W \in \mathbb{C}^{2}$, a

convenient local holomorphic trivialization of $E$. On the other hand, 
when $\vartheta \in \mathbb{Z}$, say $\vartheta=n \in \mathbb{Z}_{\leq 0}$, the pole is resonant and the matrix form may be reduced (by local gauge transformation as above) to

$$
\text { either } \quad \Omega=\left(\begin{array}{cc}
\frac{n}{2} & 0 \\
0 & -\frac{n}{2}
\end{array}\right) \frac{d z}{z}, \quad \text { or } \quad \Omega=\left(\begin{array}{cc}
\frac{n}{2} & z^{n} \\
0 & -\frac{n}{2}
\end{array}\right) \frac{d z}{z} \text {. }
$$

The point $\omega_{\infty}$ is an apparent singular point for $\nabla$ in the former case (actually regular when $n=0$ ), and a logarithmic singular point in the latter case.

The connection $\nabla$ is regular over the affine part $X^{*}=X-\left\{\omega_{\infty}\right\}$ of the curve, and we inherit a monodromy representation

$$
\rho: \pi_{1}\left(X^{*}\right) \rightarrow \mathrm{SL}(2, \mathbb{C})
$$

which is well defined by $(E, \nabla)$ up to $\mathrm{SL}(2, \mathbb{C})$-conjugacy. Fix a loop $\delta \in \pi_{1}\left(X^{*}\right)$ going to $\omega_{\infty}$, turning once around, and coming back to the base point; then $\rho(\delta)$ is the local monodromy of $(E, \nabla)$ around $\omega_{\infty}$ and is conjugated to

$$
\left(\begin{array}{cc}
e^{i \pi \vartheta} & 0 \\
0 & e^{-i \pi \vartheta}
\end{array}\right) \quad\left(\text { resp. } \pm\left(\begin{array}{ll}
1 & 1 \\
0 & 1
\end{array}\right) \text { in the logarithmic case }\right) .
$$

All of this obviously does not depend on the choice of the base point for the fundamental group. We note that the singular point $\omega_{\infty}$ is an apparent singular point if, and only if, the local monodromy $\rho(\delta)$ is $\pm I$, the center of $\operatorname{SL}(2, \mathbb{C})$; this can occur only when $\vartheta \in \mathbb{Z}^{*}$.

6.1. Riemann-Hilbert correspondence. For each exponent $\vartheta \in \mathbb{C}$, we get an analytic map

$$
\left\{\begin{array}{c}
\text { Lamé connections over } X \\
\text { with exponent } \vartheta \\
(E, \nabla)
\end{array}\right\} / \sim \stackrel{R H}{\rightarrow}\left\{\begin{array}{c}
\rho: \pi_{1}\left(X^{*}\right) \rightarrow \operatorname{SL}(2, \mathbb{C}) \\
\operatorname{trace}(\rho(\delta))=2 \cos (\pi \vartheta)
\end{array}\right\} / \sim
$$

which assigns to a Lamé connection, up to holomorphic bundle isomorphisms, the corresponding monodromy representation, up to $\mathrm{SL}(2, \mathbb{C})$ conjugacy. This map is almost one-to-one: it is surjective, and it is injective in restriction to those connections without apparent singular point, i.e. such that the local monodromy is $\rho(\delta) \neq \pm I$.

When $(E, \nabla)$ has an apparent singular point at $\omega_{\infty}$, say $\vartheta=n \in \mathbb{Z}_{>0}$, all horizontal sections have meromorphic extension at $\omega_{\infty}$; those holomorphic ones are contained in a sub-line-bundle of $E$ near $\omega_{\infty}$, say $L$; through the normal form (47), $L$ is the constant line bundle generated by $\left(\begin{array}{l}1 \\ 0\end{array}\right)$. Except in very special cases, $L$ does not extends as a line bundle $L \subset E$ on the whole of $X$ : it is only defined at the 
neighborhood of $\omega_{\infty}$. The fiber of $L$ at $\omega_{\infty}$ coincides with the eigenline of the residual matrix associated to the positive eigenvalue $\frac{n}{2}$. By the Riemann-Hilbert correspondence over the punctured curve $X^{*}$, any two Lamé connections with the same monodromy representation are conjugated by a gauge transformation over $X^{*}$; the conjugacy extends as a global gauge transformation if, and only if, it conjugates the corresponding local line bundles as defined above. One can restore the injectivity of the Riemann-Hilbert map in the following way. Consider the monodromy representation $\rho$ as an action of the fundamental group $\pi_{1}\left(X^{*}, p\right)$ on the space $E_{p} \simeq \mathbb{C}^{2}$ of germs of solutions at the base point $p$; now, dragging back, by analytic continuation along (half-) $\delta$, the local holomorphic solutions at $\omega_{\infty}$ until the base point $p$, we get a one dimensional subspace $L_{p} \subset E_{p}$. In other words, $L_{p}$ is obtained by analytic continuation of $L$ (as a $\nabla$-invariant line bundle) along $\delta$. The Lamé connection (with apparent singular point) is characterized by the pair

$$
\left(\rho, L_{p}\right) \in \operatorname{Hom}\left(\pi_{1}\left(X^{*}, p\right), S L\left(E_{p}\right)\right) \times \mathbb{P}\left(E_{p}\right)
$$

up to conjugacy:

$$
\left(\rho, L_{p}\right) \sim\left(M^{-1} \rho M, M^{-1} L_{p}\right), \quad M \in \mathrm{SL}(2, \mathbb{C}) .
$$

This is a kind of parabolic structure for the space of representations.

6.2. Fricke moduli space. Let us first recall how to describe the moduli space of representations following Fricke (see [13]). Fix standart generators $\alpha, \beta \in \pi_{1}\left(X^{*}\right)$ of the fundamental group so that the commutator $\delta:=[\alpha, \beta]=\alpha \beta \alpha^{-1} \beta^{-1}$ represents a small loop turning once around the puncture as before. We neglect the base point as it will play no role in our discussion. A representation $\rho$ is determined by the images of generators

$$
A:=\rho(\alpha) \quad \text { and } \quad B:=\rho(\beta) .
$$

The ring of polynomial functions on $\mathrm{SL}(2, \mathbb{C}) \times \mathrm{SL}(2, \mathbb{C})$ that are invariant under the $\mathrm{SL}(2, \mathbb{C})$-conjugacy action is generated by

$$
a:=\operatorname{tr}(A), \quad b:=\operatorname{tr}(B) \quad \text { and } \quad c:=\operatorname{tr}(A B) ;
$$

for instance, the trace of the commutator $\delta=[\alpha, \beta]$ is given by

$$
d:=\operatorname{tr}([A, B])=a^{2}+b^{2}+c^{2}-a b c-2 .
$$

and we have

$$
\rho \text { is reducible } \Leftrightarrow a^{2}+b^{2}+c^{2}-a b c-2=2 .
$$


The geometric quotient of $\operatorname{Hom}\left(\pi_{1}\left(X_{t}^{*}\right), \mathrm{SL}(2, \mathbb{C})\right)$ by the $\mathrm{SL}(2, \mathbb{C})$-conjugacy action identifies with $\mathbb{C}^{3}$ via the composition

$$
\begin{array}{cccc}
\operatorname{Hom}\left(\pi_{1}\left(X^{*}\right), \mathrm{SL}(2, \mathbb{C})\right) & \rightarrow \mathrm{SL}(2, \mathbb{C}) \times \mathrm{SL}(2, \mathbb{C}) & \rightarrow & \mathbb{C}^{3} \\
\rho & \mapsto & (A, B) & \mapsto(a, b, c)
\end{array}
$$

Precisely (see [9, 13]), when $a^{2}+b^{2}+c^{2}-a b c-2 \neq 2$, the fibre over $(a, b, c)$ consists in the single $\mathrm{SL}(2, \mathbb{C})$-conjugacy class of the irreducible representation defined by

$$
A=\left(\begin{array}{cc}
a & -1 \\
1 & 0
\end{array}\right) \quad \text { and } B=\left(\begin{array}{cc}
0 & \gamma^{-1} \\
-\gamma & b
\end{array}\right) \quad \text { with } \quad \gamma+\gamma^{-1}=c
$$

This normal form is obtained in any basis of the form $(v,-\gamma B . v)$ where $v$ is an eigenvector for the product $A . B$ with eigenvalue $\gamma$; it only depends on the choice of the root $\gamma$. The commutator is therefore given by

$$
[A, B]=\left(\begin{array}{cc}
-\frac{2 \gamma^{2}+1}{\gamma^{2}} & \frac{a-b \gamma}{\gamma^{2}} \\
\frac{a \gamma-b}{\gamma} & \frac{1}{\gamma^{2}}
\end{array}\right) .
$$

One can check by direct computation that matrices $A$ and $B$ above share a common eigenvector if, and only if, $d=2$.

Corollary 14. A Lamé connection is reducible if, and only if, $\vartheta \in \mathbb{Z}$.

The elliptic involution $\sigma: X \rightarrow X ;(x, y) \mapsto(x,-y)$ acts on our moduli space sending the $\operatorname{SL}(2, \mathbb{C})$-class defined by $(A, B)$ onto that one defined by $\left(A^{-1}, B^{-1}\right)$; this may be seen by choosing a fixed point of $\sigma$ as the base point for the fundamental group. One of the key point of our construction is

Lemma 15. An $\mathrm{SL}(2, \mathbb{C})$-class is stabilized by the elliptic involution $\sigma$ if, and only if, it consists in either irreducible, or abelian representations. In other words, for any pair $(A, B)$ generating an irreducible or abelian subgroup of $\mathrm{SL}(2, \mathbb{C})$, there exists $M \in \mathrm{SL}(2, \mathbb{C})$ such that

$$
M^{-1} A M=A^{-1} \quad \text { and } \quad M^{-1} B M=B^{-1} .
$$

In the irreducible case, $M$ is unique up to a sign and $\operatorname{tr}(M)=0$.

Proof. In $\mathrm{SL}(2, \mathbb{C})$, we have $\operatorname{tr}\left(A^{-1}\right)=\operatorname{tr}(A)$ and

$$
\operatorname{tr}\left(A^{-1} B^{-1}\right)=\operatorname{tr}\left(B^{-1} A^{-1}\right)=\operatorname{tr}\left((A B)^{-1}\right)=\operatorname{tr}(A B)
$$

so that the involution acts trivially on the quotient, i.e. on triples $(a, b, c)$. Since irreducible $\operatorname{SL}(2, \mathbb{C})$-classes are characterized by their corresponding triple $(a, b, c)$, they are $\sigma$-invariant. Another way to show this is to note that the matrix $M$ as in the statement has to permute the two eigenvectors of each matrix $A$ and $B$; in $\operatorname{PSL}(2, \mathbb{C})$, 
we are looking for an element $\bar{M}$ permuting the corresponding points in $\mathbb{P}^{1}$, sending a quadruple $\left(a_{1}, a_{2}, b_{1}, b_{2}\right)$ to the quadruple $\left(a_{2}, a_{1}, b_{2}, b_{1}\right)$. But the cross-ratios are the same, thus showing the existence of $\bar{M}$; moreover, $\bar{M}$ is an involution since its square fixes the 4 points, and therefore $\operatorname{tr}(M)=0$. Degenerate cases where $a_{1}=a_{2}$ or/and $b_{1}=b_{2}$ have to be treated apart; we omit this discussion. In the reducible case, we have say $a_{1}=b_{1}$ and $\bar{M}$ exists if, and only if, $a_{2}=b_{2}$, thus implying abelianity. Finally, $\sigma$ exchanges upper and lower triangular $\mathrm{SL}(2, \mathbb{C})$-representations but stabilizes diagonal ones.

When the matrices $A$ and $B$ are in the normal form above, the matrix $M$ of the previous Lemma is given, up to a sign, by

$$
M=\left(\begin{array}{cc}
\frac{\gamma^{2}-1}{2 \gamma} & \frac{a-b \gamma}{2 \gamma} \\
\frac{a \gamma-b}{2} & -\frac{\gamma^{2}-1}{2 \gamma}
\end{array}\right)
$$

We resume our discussion in the non resonant case:

Corollary 16. Given an elliptic curve $X$ and $\vartheta \notin \mathbb{Z}$, Lamé connections on $X$ with exponent $\vartheta$ are in one-to-one correspondence with the points of the smooth affine hypersurface

$$
S_{d}:=\left\{(a, b, c) \in \mathbb{C}^{3} ; a^{2}+b^{2}+c^{2}-a b c-2=d\right\}, \quad d=2 \cos (\pi \vartheta) ;
$$

they are irreducible and $\sigma$-invariant.

Proof. The connection is irreducible $(\vartheta \notin \mathbb{Z})$ and has no apparent singular point. The Riemann-Hilbert is therefore injective and assertions directly follow from Lemma 15.

6.3. Resonant cases. We now complete the picture with those resonant parameters $\vartheta \in \mathbb{Z}$.

6.3.1. $\vartheta \in \mathbb{Z} \backslash 2 \mathbb{Z}$. The monodromy representation is irreducible, characterized by the corresponding triple $(a, b, c)$. The local monodromy around $\omega_{\infty}$ is parabolic, with twice the eigenvalue -1 , and is given by the commutator

$$
[A, B]=\left(\begin{array}{cc}
a^{2}+b^{2}+\gamma^{2}-a b c & \gamma^{-2}(a-b \gamma) \\
a-b \gamma^{-1} & \gamma^{-2}
\end{array}\right), \quad \gamma+\gamma^{-1}=c
$$

we have $\operatorname{tr}([A, B])=a^{2}+b^{2}+c^{2}-a b c-2=-2$ and $[A, B]=-I$ precisely when $(a, b, c)=(0,0,0)$, the unique singular point of the surface.

Proposition 17. Lamé connections with exponent $\vartheta \in \mathbb{Z} \backslash 2 \mathbb{Z}$ having a logarithmic singular point are in one-to-one correspondence with the smooth points of the Markov affine hypersurface

$$
S_{-2}=\left\{(a, b, c) \in \mathbb{C}^{3} ; a^{2}+b^{2}+c^{2}-a b c=0\right\} ;
$$


they are irreducible and $\sigma$-invariant.

Proof. The same as for Corollary 16.

When $(a, b, c)=(0,0,0)$, the image of the monodromy representation is the order 8 dihedral group (i.e. quaternionic)

$$
A=\left(\begin{array}{cc}
0 & -1 \\
1 & 0
\end{array}\right) \text { and } B=\left(\begin{array}{cc}
0 & i \\
i & 0
\end{array}\right)
$$

and the singular point $\omega_{\infty}$ of the connection is apparent: $[A, B]=-I$. In this case, the Lamé connection is not characterized by its monodromy representation and we indeed have

Proposition 18. Lamé connections over the singular point $(a, b, c)=$ $(0,0,0)$ are in one-to-one correspondence with $\mathbb{P}^{1}$. They have the same monodromy representation into the order 8 dihedral subgroup of $\operatorname{SL}(2, \mathbb{C})$, thus irreducible; all of them are $\sigma$-invariant.

Proof. The Lamé connection is determined by its monodromy representation $\rho$, acting on the space of solutions $E_{p} \simeq \mathbb{C}^{2}$, and the line $L_{p} \subset E_{p}$ corresponding to those solutions holomorphic at $\omega_{\infty}$ after analytic continuation along $\delta$. In other words, the connection is determined by a triple

$$
(A, B, L) \in \mathrm{SL}(2, \mathbb{C}) \times \mathrm{SL}(2, \mathbb{C}) \times \mathbb{P}^{1}
$$

another triple $(\tilde{A}, \tilde{B}, \tilde{L})$ will represent a connection gauge equivalent to the initial one if, and only if

$$
(\tilde{A}, \tilde{B}, \tilde{L})=\left(M^{-1} A M, M^{-1} B M, M^{-1} L\right), \quad M \in \operatorname{SL}(2, \mathbb{C}) .
$$

The monodromy representation, being irreducible here, has centralizor $\pm I$ acting trivially on $\mathbb{P}^{1}$ : once the monodromy representation $(A, B)$ is fixed, gauge equivalence classes of connections are in one-to-one correspondence with $\mathbb{P}^{1} \supset L$.

One easily check that the action of $\sigma$ on Lamé connections induces the following action on the corresponding triples:

$$
\sigma:(A, B, L) \mapsto\left(A^{-1}, B^{-1},(A B)^{-1} L\right) .
$$

It turns out that, when $(a, b, c)=(0,0,0)$ (and for instance $\gamma=i$ ), the matrix $M$ given by Lemma 15 (see (48) ) satisfies

$$
M=A \cdot B=\left(\begin{array}{cc}
i & 0 \\
0 & -i
\end{array}\right)
$$

and thus conjugates $\sigma(A, B, L)$ to $(A, B, L)$, thus proving the $\sigma$-invariance of the corresponding connection, a kind of miracle. 
Remark 19. In fact, the moduli space of Lamé connections with fixed exponent $\vartheta \in \mathbb{Z} \backslash 2 \mathbb{Z}$ may be viewed, over the surface $S_{-2}:\left\{a^{2}+b^{2}+\right.$ $\left.c^{2}-a b c=0\right\}$, as the minimal resolution obtained after blowing-up once the singular point at $(0,0,0)$ : the exceptional divisor stands for those connections with apparent singular point considered in Proposition 18. This will immediately follow from the similar result obtained in [19] for connections over $\mathbb{P}^{1}$ after our descent construction; let us give some direct arguments. Along the smooth part of the affine surface $S_{-2}$, one can consider the one dimensional subspace $L_{p} \subset E_{p}$ of solutions holomorphic at $\omega_{\infty}$ after analytic continuation along $\delta$ : one can check from the local model (47) in the logarithmic case that $L_{p}$ coincides with the eigenspace of the local monodromy $[A, B]$, namely

$$
L=\mathbb{C} \cdot\left(\begin{array}{c}
\gamma^{2}+1 \\
-a \gamma^{2}+b \gamma
\end{array}\right)
$$

The exceptional divisor of $S_{-2}$ is given by $a^{2}+b^{2}+c^{2}=0$ in homogeneous coordinates $(a: b: c)$ and can be parametrized by

$$
\mathbb{P}^{1} \hookrightarrow \mathbb{P}^{2} ; s \mapsto\left(i\left(s^{2}+1\right): s^{2}-1: 2 s\right) .
$$

One can easily verify that the line $L_{p}$ tends to $\mathbb{C} \cdot\left(\begin{array}{l}1 \\ s\end{array}\right)$ when the representation $\rho$ tends to the point s via the parametrization above.

6.3.2. $\vartheta \in 2 \mathbb{Z}$. In this case, a combination of several non Hausdorff phenomena occur for both moduli spaces of representations, and connections. The Hausdorff quotient is given by the Cayley affine hypersurface

$$
S_{2}=\left\{(a, b, c) \in \mathbb{C}^{3} ; a^{2}+b^{2}+c^{2}-a b c=4\right\} .
$$

The singular points of $S_{2}$ are

$$
(a, b, c)=(2,2,2),(2,-2,-2),(-2,2,-2) \text { and }(-2,-2,2) ;
$$

they play the same role in the sense that they are permuted by changing signs of generators:

$$
(A, B),(A,-B),(-A, B) \text { and }(-A,-B) .
$$

Over a smooth point $(a, b, c) \in S_{2}$, there are exactly 3 distinct $\mathrm{SL}(2, \mathbb{C})$-conjugacy classes of representations, namely

$$
(A, B)=\left(\left(\begin{array}{cc}
\alpha & \lambda \\
0 & \alpha^{-1}
\end{array}\right),\left(\begin{array}{cc}
\beta & \mu \\
0 & \beta^{-1}
\end{array}\right)\right), \quad\left(\left(\begin{array}{cc}
\alpha & 0 \\
\lambda & \alpha^{-1}
\end{array}\right),\left(\begin{array}{cc}
\beta & 0 \\
\mu & \beta^{-1}
\end{array}\right)\right)
$$

(genuine upper and lower triangular) 


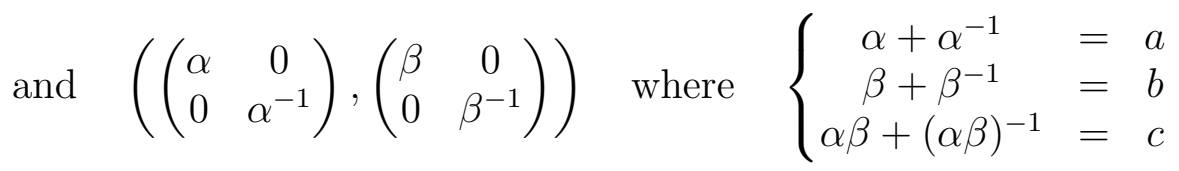

In the triangular cases, $\lambda$ and $\mu$ can be choosen arbitrarily, provided that $[A, B] \neq I$, i.e. $b \lambda+a \mu \neq 0$.

Each of the two triangular representations correspond to a unique Lamé connection (with a logarithmic singular point). They are permuted by $\sigma$ and thus not $\sigma$-invariant. The moduli space of those triangular connections is a 2-fold cover of the smooth part $S_{2}^{*}$ of $S_{2}$, the two sheets of which are permuted around each of the four singular points.

When $\vartheta=0$, the diagonal representation corresponds to a unique (regular) Lamé connection which is $\sigma$-invariant.

When $\vartheta \neq 0$, Lamé connections over the diagonal representation have an apparent singular point: there are exactly 3 equivalence classes corresponding to the following choices for the line bundle $L$ (see the proof of Proposition [18)

$$
\mathbb{P}^{1} \ni L=(1: 0),(0: 1) \quad \text { or } \quad(1: 1)
$$

(any choice $L=(1: s), s \in \mathbb{C}^{*}$, is equivalent to $L=(1: 1)$ ). The involution $\sigma$ permutes the two first connections while it fixes the "generic" third one: in both situations, it suffices to choose the matrix

$$
M=\left(\begin{array}{cc}
0 & i \gamma^{-1} \\
i \gamma & 0
\end{array}\right)
$$

(see again proof of Proposition 18). By the way, Lamé connections with diagonal monodromy split into another two-fold cover of $S_{2}^{*}$, with Galois involution $\sigma$, and a copy of $S_{2}^{*}$, on which $\sigma$ acts trivially.

Finally, over each smooth point $(a, b, c) \in S_{2}^{*}$, there are exactly 5 Lamé connections (resp. 3 when $\vartheta=0$ ), only one of which is $\sigma$ invariant.

Consider now a singular point, say $(a, b, c)=(2,2,2)$ (recall that the four singular points play the same role). Above this point, there are infinitely many distinct $\mathrm{SL}(2, \mathbb{C})$-conjugacy classes in the fibre defined by parabolic pairs

$$
(A, B)=\left(\left(\begin{array}{ll}
1 & r \\
0 & 1
\end{array}\right),\left(\begin{array}{ll}
1 & s \\
0 & 1
\end{array}\right)\right), \quad(r: s) \in \mathbb{P}^{1}
$$

and the central one $(A, B)=(I, I)$ (when $(r, s)=(0,0))$. When $\vartheta=0$, those representations bijectively correspond to Lamé connections that are $\sigma$-invariant. When $\vartheta \neq 0$, then for each parabolic representation, there are exactly 2 Lamé connections given by $L=(1: 0)$ and $L=(s$ : 
1) (any $s \in \mathbb{C}$ are equivalent) and one Lamé connection with trivial monodromy; all of them are $\sigma$-invariant.

Remark 20. When we have an apparent singular point and the direction $L$ is fixed by the monodromy, we get a $\nabla$-invariant line bundle, say $L$ again, having positive degree, thus implying unstability of the bundle $E$. Indeed, in this case we have $\vartheta= \pm 2 m, m \in \mathbb{Z}_{>0}$ and $\nabla$ horizontal sections in $L$ are holomorphic, vanishing at the order $m$ at $\omega_{\infty}$; by Fuchs relation, $\operatorname{deg}(L)=m$. We do not want to consider this kind of deformations in this paper.

We resume a part of our discussion

Proposition 21. When $\vartheta=2 m \in \mathbb{Z}$, all semistable and $\sigma$-invariant Lamé connections have an apparent singular point (resp. regular when $m=0$ ) at $\omega_{\infty}$ and their monodromy data belong to the following list:

$$
\begin{aligned}
\text { - }(A, B) & =\left(\left(\begin{array}{cc}
\alpha & 0 \\
0 & \alpha^{-1}
\end{array}\right),\left(\begin{array}{cc}
\beta & 0 \\
0 & \beta^{-1}
\end{array}\right)\right) \text { with }(\alpha, \beta) \neq( \pm 1, \pm 1) \\
\text { and } L & =\left(\begin{array}{l}
1 \\
1
\end{array}\right) \text { when } m \neq 0 \\
\text { - }(A, B) & =\left( \pm\left(\begin{array}{ll}
1 & s \\
0 & 1
\end{array}\right), \pm\left(\begin{array}{ll}
1 & t \\
0 & 1
\end{array}\right)\right) \text { with }(s, t) \in \mathbb{P}^{1} \\
\text { and } L & =\left(\begin{array}{l}
1 \\
1
\end{array}\right) \text { when } m \neq 0 .
\end{aligned}
$$

When $m=0$, we also have to add the 4 connections with monodromy $(A, B)=( \pm I, \pm I)$.

6.4. Irreducible Lamé connections are elliptic pull-back. We now check that $\sigma$-invariant representations are actually coming from representations of the 4 -punctured sphere via the elliptic cover. Precisely, let us consider the elliptic pull-back construction of section 2 from the monodromy representation point of view. For a connection $(E, \nabla) \in \mathcal{M}_{t}^{\boldsymbol{\theta}}$ with exponents

$$
\boldsymbol{\theta}=\left(\frac{1}{2}, \frac{1}{2}, \frac{1}{2}, \frac{1}{2}+\frac{\vartheta}{2}\right)
$$

consider the monodromy representation

$$
\pi_{1}\left(\mathbb{P}^{1} \backslash\{0,1, t, \infty\}\right) \rightarrow \mathrm{SL}(2, \mathbb{C}) .
$$

It is defined by matrices $\left(M_{0}, M_{1}, M_{t}, M_{\infty}\right)$ satisfying

$$
\left(M_{0}\right)^{2}=\left(M_{1}\right)^{2}=\left(M_{t}\right)^{2}=-I \quad \text { and } \quad \operatorname{tr}\left(M_{\infty}\right)=-2 \sin \left(\frac{\pi \vartheta}{2}\right)
$$


The monodromy of its elliptic pull-back is therefore given by

$$
A=M_{0} M_{1} \quad \text { and } \quad B=M_{1} M_{t}
$$

(see [7, section 2] for details), the commutator by

$$
[A, B]=-M_{0}\left(M_{\infty}\right)^{2} M_{0}^{-1}
$$

and we can check that its trace is given by

$$
\operatorname{tr}([A, B])=-\operatorname{tr}\left(\left(M_{\infty}\right)^{2}\right)=2-\left(\operatorname{tr}\left(M_{\infty}\right)\right)^{2}=2 \cos (\pi \vartheta) .
$$

Clearly, this representation is $\sigma$-invariant since for $M:= \pm M_{1}$ we get from (49) that

$$
M^{-1} A M=A^{-1} \quad \text { and } \quad M^{-1} B M=B^{-1} .
$$

Conversely, let $(A, B)$ defines the monodromy of a $\sigma$-invariant Lamé connection: there is a matrix $M$ conjugating $(A, B)$ to $\left(A^{-1}, B^{-1}\right)$. From the previous sections, it is clear that we can assume that $M$ has null trace: $M^{2}=-I$. Then it is straightforward to check that $(A, B)$ is the elliptic pull-back of the following represention

$$
M_{0}=-A M, \quad M_{1}=M, \quad M_{t}=-M B \quad \text { and } \quad M_{\infty}=B^{-1} M A^{-1} .
$$

When the monodromy $(A, B)$ is irreducible, then $\left(M_{0}, M_{1}, M_{t}, M_{\infty}\right)$ is the unique quadruple up to a sign, whose elliptic pull-back gives the representation $(A, B)$.

Corollary 22. Let $(E, \nabla)$ be an irreducible Lamé connection with exponent $\vartheta \notin 2 \mathbb{Z}$. Then there is a unique (up to isomorphism) connection $(\underline{E}, \underline{\nabla}) \in \mathcal{M}_{t}^{\boldsymbol{\theta}}$ with exponents

$$
\boldsymbol{\theta}=\left(\frac{1}{2}, \frac{1}{2}, \frac{1}{2}, \frac{1}{2}+\frac{\vartheta}{2}\right)
$$

such that $(E, \nabla)$ is the elliptic pull-back of $(\underline{E}, \underline{\nabla})$.

Proof. Consider $(A, B)$ the monodromy representation of $(E, \nabla)$. There is a unique quadruple $\left(M_{0}, M_{1}, M_{t}, M_{\infty}\right)$ lifting to the representation $(A, B)$ such that $\operatorname{tr}\left(M_{\infty}\right)=-2 \sin \left(\frac{\pi \vartheta}{2}\right)$ (one has to conveniently choose the sign of $M$, and therefore of the quadruple). Assume non resonance condition $\vartheta \notin \mathbb{Z}$. By the Riemann-Hilbert correspondance, there is a unique connection $(\underline{E}, \underline{\nabla}) \in \mathcal{M}_{t}^{\boldsymbol{\theta}}$ with prescribed monodromy and exponents up to isomorphism. By construction, the elliptic pull-back of $(\underline{E}, \underline{\nabla})$ must have exponent $\vartheta$ and holonomy representation $(A, B)$, the same as $(E, \nabla)$. Again by (unicity part of) Riemann-Hilbert correspondance, the elliptic pull-back of $(\underline{E}, \underline{\nabla})$ must be isomorphic to $(E, \nabla)$, proving the Corollary in the non resonant case. When $\vartheta \in \mathbb{Z} \backslash 2 \mathbb{Z}$, the proof is the same if the singular point is logarithmic (i.e. with infinite monodromy). However when the pole of $(E, \nabla)$ becomes apparent, 
then we have to deal with a parabolic structure to restore injectivity of the Riemann-Hilbert correspondance; we do not detail, but the key step of the proof is precisely given by Proposition 18 .

\section{Proof of Theorem 1}

We now detail the proof of Theorem 1, Let $t \mapsto\left(E_{t}, \nabla_{t}\right)$ be an isomonodromic deformation of an irreducible Lamé connection with exponent $\vartheta$. From Corollary 22, it is the elliptic pull-back of an isomonodromic deformation $t \mapsto\left(\underline{E}_{t}, \underline{\nabla}_{t}\right) \in \mathcal{M}_{t}^{\boldsymbol{\theta}}$ with $\boldsymbol{\theta}=\left(\frac{1}{2}, \frac{1}{2}, \frac{1}{2}, \frac{1}{2}+\frac{\vartheta}{2}\right)$. From Bolibrukh transversality (see section 5.10), there is an open set of the parameter for which the bundle $E_{t}$ is trivial and the parabolic directions $\left(l_{0}, l_{1}, l_{t}, l_{\infty}\right)$ are pairwise distinct; moreover, they do not lie on a degree $(1,1)$ curve. Therefore, the cross-ratio

$$
c=\frac{l_{t}-l_{0}}{l_{1}-l_{0}} \frac{l_{1}-l_{\infty}}{l_{t}-l_{\infty}} \in \mathbb{P}^{1} \backslash\{0,1, t, \infty\}
$$

is not special and we can apply Proposition 7 and Corollary 10 and get the explicit expression for $\mathrm{Tu}$ invariant

$$
\lambda\left(E_{t}\right)=q+\frac{\rho+\kappa_{\infty}}{p}
$$

where $t \mapsto(p(t), q(t))$ are the invariants of $\left(\underline{E}_{t}, \underline{\nabla}_{t}\right)$. In particular, $\lambda\left(E_{t}\right)$ coincide with the Okamoto symmetric $s_{2} s_{1} s_{2}$ of $q(t)$ (see section 5.8) which is therefore a Painlevé VI solution itself, for parameters

$$
\tilde{\boldsymbol{\kappa}}=\left(\frac{\vartheta}{4}, \frac{\vartheta}{4}, \frac{\vartheta}{4}, \frac{\vartheta}{4}\right) .
$$

\section{Flat Logarithmic $\operatorname{sl}(2, \mathbb{C})$-CONnECtions}

Here, we recall basic facts about flat logarithmic connections that can be found in greater details in [10, 31, 24, 16].

A meromorphic connection of rank $r$ over a smooth complex manifold $X$ is a pair $(E, \nabla)$ where $E$ is a locally trivial rank $r$ holomorphic vector bundle over $X$ and $\nabla$ a $\mathbb{C}$-linear morphism of sheaves

$$
\nabla: \mathcal{E} \rightarrow \mathcal{M}(K) \otimes_{\mathcal{O}} \mathcal{E}
$$

(where $\mathcal{E}$ is the sheaf of holomorphic sections of $E$, and $\mathcal{M}(K)$, the sheaf of meromorphic sections of the canonical bundle $K$ ) satisfying moreover the Leibniz rule

$$
\nabla(f v)=d f \otimes v+f \nabla(v)
$$


for all sections $f$ and $v$ of the structural sheaf $\mathcal{O}$ and the vector bundle $E$ respectively. From the analytic point of view, $E$ is defined by charts

$$
U_{i} \times \mathbb{C}^{r} \ni\left(x, Y_{i}\right), \quad X=\cup_{i} U_{i},
$$

glued by transition maps

$$
Y_{i}=M_{i, j} Y_{j}, \quad M_{i, j} \in \mathrm{GL}\left(r, \mathcal{O}\left(U_{i} \cup U_{j}\right)\right) ;
$$

then $\nabla$ is a differential operator of the form

$$
Y_{i} \mapsto d Y_{i}-\Omega_{i} Y_{i}, \quad \Omega_{i} \in \operatorname{gl}\left(r, \mathcal{M}(K)\left(U_{i}\right)\right),
$$

in trivializing charts, satisfying compatibility conditions

$$
\Omega_{j}=M_{i, j}^{-1} \Omega_{i} M_{i, j}+M_{i, j}^{-1} d M_{i, j} .
$$

Throughout this work, we will adopt the later analytic point of view. Meromorphic connections $(E, \nabla)$ will be considered up to holomorphic isomorphisms of vector bundles.

8.1. Polar divisor. We say that $\nabla$ has a pole at some point $x \in U_{i} \subset$ $X$ if at least one of the coefficients of the corresponding matrix $\Omega_{i}$ has a pole at $x$; the order of the pole is therefore given by the maximal order for all coefficients. One easily check that is does not depend neither on the choice of the chart $U_{i}$, nor of the local trivialization $Y_{i}$. The polar divisor $D=(\nabla)_{\infty}$ of the connection is a well defined positive divisor on $X$.

\subsection{Flatness and monodromy representation. A horizontal sec-} tion (or solution) of $(E, \nabla)$ is any section $v$ of $E$ satisfying $\nabla(v)=0$; in a chart, horizontal sections $Y_{i}(x)$ are the solutions of the Pfaffian system $d Y_{i}=\Omega_{i} Y_{i}$. The connection $(E, \nabla)$ is flat (or integrable) when it satisfies

$$
d \Omega_{i}+\Omega_{i} \wedge \Omega_{i}=0
$$

in any chart (in once chart is actually enough). This is equivalent to the existence of a basis $\mathcal{B}=\left(v_{1}, \ldots, v_{r}\right)$ of horizontal holomorphic sections at any regular point for $\nabla$. In other words, the connection is flat if it is locally trivial at any regular point, i.e. given by $Y_{i} \mapsto d Y_{i}$ $\left(\Omega_{i} \equiv 0\right)$ in convenient local trivialisation of $E$. This basis $\mathcal{B}$ admits analytic continuation along any paths in $X \backslash D$, just by gluing local trivializations of $\nabla$ with help of transition maps of the bundle. Therefore, fixing $x_{0} \in X \backslash D$ and a basis $\mathcal{B}$ like above at the neighborhood of $x_{0}$, we get the monodromy representation of $(E, \nabla)$ with respect to $\mathcal{B}$ a homomorphism

$$
\rho_{\nabla, \mathcal{B}}: \pi_{1}\left(X \backslash D, x_{0}\right) \rightarrow \mathrm{GL}(r, \mathbb{C}) ; \gamma \mapsto M_{\gamma}
$$


defined as follows: if $\mathcal{B}_{\gamma}$ is the new basis of horizontal sections around $x_{0}$ obtained after analytic continuation along $\gamma$, then $M_{\gamma}$ is given by

$$
\mathcal{B}_{\gamma}=\mathcal{B} M_{\gamma}
$$

If we change the basis of horizontal sections $\mathcal{B}$ by another one $\mathcal{B}^{\prime}=M \mathcal{B}$, $M \in \mathrm{GL}(r, \mathbb{C})$, then the new monodromy representation is given by

$$
\rho_{\nabla, \mathcal{B}^{\prime}}(\gamma)=M \cdot \rho_{\nabla, \mathcal{B}^{\prime}}(\gamma) \cdot M^{-1}, \quad \forall \gamma \in \pi_{1}\left(X \backslash D, x_{0}\right) .
$$

Therefore, the monodromy representation is well-defined by $\nabla$ up to $\mathrm{GL}(r, \mathbb{C})$-conjugacy and we will simply denote by $\rho_{\nabla}$ any representative of the conjugacy class.

8.3. Flat logarithmic connections. A flat connection is said logarithmic when it has only simple poles (i.e. $D$ is reduced) and moreover, in charts, the matrix connection $\Omega$ is such that its differential $d \Omega$ has simple poles as well. This later condition is equivalent to the fact that, at the neighborhood any smooth point of the polar divisor $D$, the connection has a product structure: there exists local coordinates $\left(x_{1}, \ldots, x_{n}\right)$ on $X$ and a local trivialization $Y_{i}$ such that $D$ is defined by $x_{2}=\cdots=x_{n}=0$ and the matrix connection only depend on the single variable $x:=x_{1}$. Therefore, along each irreducible component of $D$, the $\operatorname{GL}(r, \mathbb{C})$-conjugacy class of the residual matrix is constant: one can talk about the eigenvalues $\left\{\theta_{1}, \ldots, \theta_{r}\right\}$ of the connection at each pole, i.e. each component of $D$. A pole is said resonant when at least two eigenvalues differ by an integer: $\theta_{i}-\theta_{j} \in \mathbb{Z}, i \neq j$. At any smooth point of a non resonant pole, the matrix connection can be further reduced to its principal part

$$
\Omega=\left(\begin{array}{ccc}
\theta_{1} & & 0 \\
& \ddots & \\
0 & & \theta_{r}
\end{array}\right) \frac{d x}{x} .
$$

In the resonant case, for each $\theta_{i}-\theta_{j} \in \mathbb{Z}_{\geq 0}$, the $(i, j)$-coefficient of $\Omega$ can be reduced to a resonant monomial $c \cdot x^{\theta_{i}-\theta_{j}} \cdot \frac{d x}{x}$. For each irreducible component $D_{j}$ of the divisor $D$, fix a path $\delta_{j}$ in $X \backslash D$ joining the base point $x_{0}$ to a smooth point of $D_{j}$. Now, consider a loop $\gamma_{j}$ in $X \backslash D$ based at $x_{0}$ going first along $\delta_{j}$ very close to $D_{j}$, turning once around $D_{j}$, and going back to $x_{0}$ by $\delta_{j}^{-1}$. The conjugacy class of $\rho\left(\gamma_{j}\right)$ does not depend on the choices and is called the local monodromy of $(E, \nabla)$ around $D_{j}$; eigenvalues are given by $\left\{e^{2 i \pi \theta_{1}}, \ldots, e^{2 i \pi \theta_{r}}\right\}$. If the local monodromy is diagonalisable, so is the residual matrix; the converse is not true. 
8.4. Trace and twist. The trace of a connection $(E, \nabla)$ is the rank 1 meromorphic connection $(\operatorname{det}(E), \operatorname{tr}(\nabla))$ where $\operatorname{det}(E)$ is the determinant of $E$ defined (with notations above) by transition charts $\operatorname{det}\left(M_{i, j}\right)$, and $\operatorname{tr}(\nabla)$, defined by $y_{i} \mapsto d y_{i}-\operatorname{tr}\left(\Omega_{i}\right) y_{i}$. We say that the connection $(E, \nabla)$ is trace free when its trace is the trivial connection (on the trivial bundle): $y \mapsto d y$. The polar divisor of the trace is bounded by that one of the initial connection. The twist of $(E, \nabla)$ by a rank 1 connection $(L, \zeta)$ is the rank $r$ connection given by their tensor product $(L \otimes E, \zeta \otimes \nabla)$ : if $(L, \zeta)$ is defined in the same open covering $U_{i}$ by $y_{i} \mapsto d y_{i}-\omega_{i} y_{i}$ with transition charts $y_{i}=m_{i, j} y_{j}$, then the twist has the matrix form $\Omega_{i}+\omega_{i} \cdot I$ with transition charts $Y_{i}=m_{i} \cdot M_{i} Y_{j}$. We have

$\operatorname{det}(L \otimes E)=\operatorname{det}(L)^{\otimes r} \otimes \operatorname{det}(E) \quad$ and $\quad \operatorname{tr}(\zeta \otimes \nabla)=\operatorname{tr}(\zeta)^{\otimes r} \otimes \operatorname{tr}(\nabla)$.

The trace of a flat (resp. logarithmic) connection is flat (resp. logarithmic).

8.5. $\operatorname{sl}(2, \mathbb{C})$-connections. For the sake of notations, we now restrict ourselves to flat logarithmic $\mathrm{sl}(2, \mathbb{C})$-connections (i.e. rank 2 and trace free); the monodromy representation takes values into $\mathrm{SL}(2, \mathbb{C})$. For each irreducible component $D_{j}$ of the polar divisor $D$, the exponent $\theta_{j} \in \mathbb{C}$, defined up to a sign, is the difference between the two eigenvalues $\pm \frac{\theta_{j}}{2}$ of the residual matrix: the corresponding local monodromy has trace $2 \cos (\pi \theta)$. The component $D_{j}$ is resonant if, and only if, $\theta_{j} \in \mathbb{Z}$. In this case, say $\theta_{j}=n \in \mathbb{Z}_{\leq 0}$, the connection matrix can be reduced to

$$
\text { either } \quad \Omega=\left(\begin{array}{cc}
\frac{n}{2} & 0 \\
0 & -\frac{n}{2}
\end{array}\right) \frac{d x}{x}, \quad \text { or } \quad \Omega=\left(\begin{array}{cc}
\frac{n}{2} & x^{n} \\
0 & -\frac{n}{2}
\end{array}\right) \frac{d x}{x}
$$

at the neighborhood of any smooth point of $D_{j}$. The corresponding local monodromy is respectively

$$
\pm\left(\begin{array}{ll}
1 & 0 \\
0 & 1
\end{array}\right), \quad \text { or } \quad \pm\left(\begin{array}{ll}
1 & 1 \\
0 & 1
\end{array}\right)
$$

where $\pm:=(-1)^{n}$. The pole is called apparent in the former case (there is no pole when $n=0$ ) and logarithmic in the later case. In any case, we note that bounded solutions

$$
v=\left(\begin{array}{c}
c x^{n / 2} \\
0
\end{array}\right), \quad c \in \mathbb{C},
$$

form a one-dimensional subspace of the space of solutions. 
8.6. Riemann-Hilbert correspondence. One defines the RiemannHilbert correspondence as the map

$$
\left\{\begin{array}{c}
\text { Flat logarithmic } \\
\operatorname{sl}(2, \mathbb{C}) \text {-connections over } X \\
\text { with polar divisor } D \\
\text { and exponent } \theta_{j} \text { over } D_{j}
\end{array}\right\} / \sim \rightarrow\left\{\begin{array}{c}
\text { Representations } \\
(E, \nabla)
\end{array} \quad \begin{array}{c}
R H \\
\pi_{1}\left(X \backslash D, x_{0}\right) \rightarrow \operatorname{SL}(2, \mathbb{C}) \\
\text { having trace } 2 \cos \left(\pi \theta_{j}\right) \\
\text { around } D_{j} \\
\rho
\end{array}\right\} / \sim
$$

which assigns to a connection, up to holomorphic bundle isomorphism, the corresponding monodromy representation, up to conjugacy. This map is surjective provided that $D$ has normal crossings [10] or $X$ has dimension $\leq 2$ [24]. It is moreover injective provided that none of the exponent is a non zero integer. In fact, the lack of injectivity comes from apparent singular points. One can restore the injectivity in the resonant case by enriching the monodromy data as follows. For each $\theta_{j} \in \mathbb{Z} \backslash\{0\}$, consider, in the basis of solutions $\mathcal{B}$ near $x_{0}$, the onedimensional subspace $L_{j} \subset \mathbb{C}^{2}$ of those solutions that are bounded around $D_{j}$ after analytic continuation along the path $\delta_{j}$. The full monodromy data, characterizing the connection up to isomorphism, is now given by the monodromy representation $\rho$ and the collection $L_{j} \in \mathbb{P}^{1}\left(=\mathbb{P}\left(\mathbb{C}^{2}\right)\right)$ where $j$ spans over the set $J^{r e s}$ of all indices such that $\theta_{j} \in \mathbb{Z} \backslash\{0\}$. Any base change $\mathcal{B}^{\prime}=M \mathcal{B}, M \in \mathrm{SL}(2, \mathbb{C})$, yields new monodromy data

$$
\rho^{\prime}=M \cdot \rho \cdot M^{-1} \quad \text { and } \quad L_{j}^{\prime}=M \cdot L_{j}, \forall j \in J^{r e s} .
$$

(for the standart action of $\operatorname{SL}(2, \mathbb{C})$ on $\mathbb{C}^{2}$ ).

Proposition 23. Assume $D$ is normal crossing reduced divisor, and $D_{j}, \gamma_{j}, \delta_{j}$ be as above. The set of flat logarithmic $\operatorname{sl}(2, \mathbb{C})$-connections $(E, \nabla)$ with polar divisor $D$ and exponents $\theta_{j}$ along $D_{j}$ modulo isomorphism is in one-to-one correspondence with the set of pairs $\left(\rho,\left(L_{j}\right)_{j \in J^{r e s}}\right)$ where

- $\rho \in \operatorname{Hom}\left(\pi_{1}\left(X \backslash D, x_{0}\right), \operatorname{SL}(2, \mathbb{C})\right)$ such that $\operatorname{tr}\left(\rho\left(\gamma_{j}\right)\right)=2 \cos \left(\pi \theta_{j}\right)$ for all $j \in J$,

- $L_{j} \in \mathbb{P}^{1}$ is $\rho\left(\gamma_{j}\right)$-invariant for all $j \in J^{\text {res }}$

modulo the $\mathrm{SL}(2, \mathbb{C})$-action given by (50).

8.7. Reducible $\mathrm{gl}(2, \mathbb{C})$-connections. A sub line bundle $L \subset E$ is said $\nabla$-invariant when it is generated by $\nabla$-horizontal sections. In this case, the connection $\nabla$ induces a meromorphic connection $\left.\nabla\right|_{L}$ on $L$. The connection $(E, \nabla)$ is said reducible when it admits such an invariant line bundle, and irreducible if not. When the connection 
is reducible, then the monodromy representation is itself reducible: the monodromy group has a common eigenvector. In the logarithmic case with normal crossing polar divisor, the converse is true: $(E, \nabla)$ is reducible if, and only if, $\rho$ is.

8.8. Projective $\mathrm{sl}(2, \mathbb{C})$-connections and Riccati foliation. A rank 2 meromorphic connection $(E, \nabla)$ induces a projective $\mathrm{sl}(2, \mathbb{C})$-connection $(\mathbb{P}(E), \mathbb{P}(\nabla))$ on $X$. If the linear connection is given in the trivializing chart $Y_{i}$ by

$$
Y_{i} \mapsto d Y_{i}-\Omega_{i} Y_{i}, \quad \Omega_{i}=\left(\begin{array}{cc}
\alpha_{i} & \beta_{i} \\
\gamma_{i} & \delta_{i}
\end{array}\right)
$$

where $\alpha_{i}, \beta_{i}, \gamma_{i}$ and $\delta_{i}$ are meromorphic 1-forms on $U_{i}$, then the projective connection $\mathbb{P}(\nabla)$ is defined in the projective trivializing chart $\mathbb{P}\left(Y_{i}\right)=\left(1: z_{i}\right) \in \mathbb{P}^{1}$ by

$$
z_{i} \mapsto d z_{i}+\beta_{i} z_{i}^{2}+\left(\alpha_{i}-\delta_{i}\right) z_{i}-\gamma_{i}
$$

Another linear connection $\left(E^{\prime}, \nabla^{\prime}\right)$ will define the same projective connection if and only if it is the twist of $(E, \nabla)$ by some rank 1 meromorphic connection $(L, \zeta)$.

Conversely, when $\mathrm{H}^{2}\left(X, \mathcal{O}^{*}\right)=0$ (as it so happens when $X$ is a curve), any $\mathbb{P}^{1}$-bundle $P$ is the projectivization of a rank 2 vector bundle $P=\mathbb{P}(E)$. Moreover, one immediately deduce from the formula above that given any meromorphic projective $\operatorname{sl}(2, \mathbb{C})$-connection on $P$ and any meromorphic (linear) connection $\zeta$ on the line bundle $L=\operatorname{det}(E)$, there is a unique meromorphic linear connection $\nabla$ on $E$ lifting the projective one on $\mathbb{P}(E)$ with prescribed trace $\operatorname{tr}(\nabla)=\zeta$ on $\operatorname{det}(E)$.

When $X$ is a curve, there are two topological types of $\mathbb{P}^{1}$-bundles : the topological type of $\mathbb{P}(E)$ is given by the class of $\operatorname{deg}(E) \in \mathbb{Z} / 2 \mathbb{Z}$. We note that topological triviality is the condition for the existence of a square root $L$ of $\operatorname{det}(E) \in \operatorname{Pic}(X)$. In other words, a $\mathbb{P}^{1}$-bundle $P$ is topologically trivial if, and only if, $P$ can be lifted as an $\operatorname{SL}(2, \mathbb{C})$ vector bundle : setting $E:=E \otimes L^{\otimes(-2)}$, we get $P=\mathbb{P}(E)$ with $\operatorname{det}(E)=\mathcal{O}$. The $\operatorname{SL}(2, \mathbb{C})$-lifting depends on the choice of a square root : it is well defined up to order two points in $\operatorname{Pic}(X)$ and there are $2^{2 g}$ possible liftings over $X$ of genus $g$. Finally, any meromorphic projective connection on $P$ lifts uniquely as a linear $\mathrm{sl}(2, \mathbb{C})$-connection $\nabla$ on $E$ (with the same polar divisor).

When $X$ is a curve, the total space of $\mathbb{P}(E)$ is a ruled surface $S \rightarrow X$ and the Riccati equation $\mathbb{P}(\nabla)=0$ defines a singular foliation $\mathcal{F}$ on $S$ whose leaves are the graphs of horizontal sections of the projective connection. The pair $(S, \mathcal{F})$ is called a Riccati foliation (see [6]). The foliation is regular, transversal to the ruling outside the polar locus of 
$\nabla$. Over the poles of the projective connection, the $\mathbb{P}^{1}$ fibre is the disjoint union of a vertical leaf of $\mathcal{F}$ and 1 or 2 singular points. Precisely, when $\nabla$ is logarithmic (simple poles), singular points correspond to the eigenlines of the linear connection $\nabla$. If $\theta$ and $\theta^{\prime}$ are the eigenvalues of $\nabla$ at some pole $x \in X$, denote by $l$ and $l^{\prime}$ the corresponding eigenlines; the exponent (or Camacho-Sad index of the vertical leaf) at the singular point $l$ of the Riccati foliation is $\kappa=\theta^{\prime}-\theta$. Let $\sigma: X \rightarrow S$ be a section.

Proposition 24. Let $X$ be a curve, $(S, \mathcal{F})$ a Riccati foliation with simple poles over $X$, and $\sigma$ a $\mathcal{F}$-invariant section. Then

$$
\sigma \cdot \sigma=\sum_{i} \kappa_{i}
$$

where $\kappa_{i}$ are the exponents of the singular points $\sigma$ passes through where $i$ runs over the invariant fibres of $\mathcal{F}$.

This is a particular case of Camacho-Sad formula (see [6], page 37).

Proof. Viewed as a projective connection, there exists a unique lifting $(E, \nabla)$ of the projective connection such that the $\nabla$-invariant line bundle $L$ corresponding to $\sigma$ is the trivial bundle, and the connection induced by $\nabla$ on $L$ is the trivial connection: the eigenvalues of $\nabla$ over the pole $i$ are given by 0 and $\kappa_{i}$. Then, Fuchs relations give

$$
\operatorname{deg}(E)=\sum_{i} \kappa_{i}
$$

and we have

$$
\sigma \cdot \sigma=\operatorname{deg}(E)-2 \operatorname{deg}(L)=\operatorname{deg}(E)
$$

Proposition 25. Let $X$ be a curve of genus $g,(S, \mathcal{F})$ a Riccati foliation over $X$ with $n$ poles (counted with multiplicity), and $\sigma: X \rightarrow S$ a section which is not $\mathcal{F}$-invariant. Then the number of tangencies between $\sigma$ and the foliation (including singular points lying on $\sigma$ ) is given by

$$
\operatorname{tang}(\mathcal{F}, \sigma)=2 g-2+n+\sigma \cdot \sigma
$$

(counted with multiplicities).

This is a particular case of Proposition 2, page 37 in [6].

Proof. Choose any lifting $(E, \nabla)$ of the projective connection and apply Lemma 6 to the line bundle $L \subset E$ corresponding to $\sigma$. 
8.9. Stability of bundle and connections. A rank 2 vector bundle over a curve $X$ is said stable (resp. semistable) when we have

$$
\operatorname{det}(E)-2 \operatorname{det}(L)>0 \quad(\text { resp. } \geq 0)
$$

for all sub line bundle $L \subset E$. This notion is invariant by projective equivalence: the $\mathbb{P}^{1}$-bundle $\mathbb{P}(E)$ is stable (resp. semistable) if

$$
\sigma \cdot \sigma>0 \quad(\text { resp. } \geq 0)
$$

for all section $\sigma: X \rightarrow \mathbb{P}(E)$.

Similarly, we say that a connection $(E, \nabla)$ is stable (resp. semistable) when

$$
\operatorname{det}(E)-2 \operatorname{det}(L)>0 \quad(\text { resp. } \geq 0)
$$

for all $\nabla$-invariant sub line bundle $L \subset E$. Again, this notion is invariant by projective equivalence: the projective connection $(\mathbb{P}(E), \mathbb{P}(\nabla))$ is stable (resp. semistable) if

$$
\sigma \cdot \sigma>0 \quad(\text { resp. } \geq 0)
$$

for all $\mathbb{P}(\nabla)$-invariant section $\sigma$. In particular, an irreducible connection $(E, \nabla)$ is stable even if the bundle $E$ is unstable. However, for a semistable connection, the bundle $E$ cannot be arbitrarily unstable: by Lemma 6 , the stability index is bounded by

$$
\operatorname{det}(E)-2 \operatorname{det}(L) \geq 2-2 g-\operatorname{deg}(D)
$$

where $D$ is the polar divisor of $\nabla$.

8.10. Meromorphic and elementary gauge tranformations. We start recalling what is an elementary transformation of a rank 2 vector bundle, say $E$, over a curve $X$. Given a point $p \in X$ and a linear subspace $l \in \mathbb{P}\left(E_{p}\right)$ in the fibre over $p$, one usually defines two birational bundle transformations

$$
\operatorname{elm}_{p, l}^{+}: E \rightarrow E^{+} \text {and } \operatorname{elm}_{p, l}^{-}: E \rightarrow E^{-},
$$

that are unique up to post-composition by a bundle isomorphism. In restriction to the punctured curve $X^{*}=X \backslash\{p\}$, both $\lim _{p, l}^{ \pm}$induce isomorphisms. At the neighborhood of $p$, they can be described as follows. Choose a local coordinate $x: U \rightarrow \mathbb{C}$ at $p$ together with a trivialization of $Y:\left.E\right|_{U} \rightarrow \mathbb{C}^{2}$ for which the linear subspace $l$ is spanned by $Y=\left(\begin{array}{l}0 \\ 1\end{array}\right)$. This, in particular, induces a trivialization 
of $\left.E\right|_{X^{*}}$ on $U^{*}=U \backslash\{p\}$. Elementary transformations $\operatorname{elm}_{p, l}^{ \pm}$can be defined by the following commutative diagram

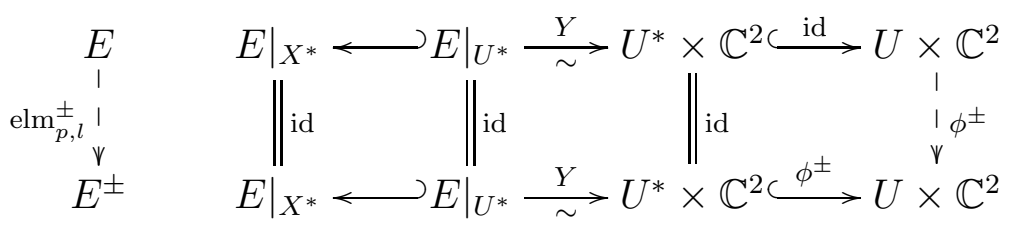

where

$$
\phi^{+}(Y)=\left(\begin{array}{ll}
1 & 0 \\
0 & x
\end{array}\right) Y \quad \text { and } \quad \phi^{-}(Y)=\left(\begin{array}{cc}
1 / x & 0 \\
0 & 1
\end{array}\right) Y
$$

All three bundles $E$ and $E^{ \pm}$are constructed by gluing the local trivial bundle $U \times \mathbb{C}^{2}$ to the same restricted bundle $\left.E\right|_{X^{*}}$ through different bundle isomorphisms (either the identity, or $\phi^{ \pm}$) over the punctured neighborhood $U^{*}$. Isomorphisms $\left.\left.E\right|_{X^{*}} \rightarrow E^{ \pm}\right|_{X^{*}}$ given by this construction extend as birational bundle transformations. We have

$$
\operatorname{det}\left(E^{ \pm}\right)=\operatorname{det}(E) \otimes \mathcal{O}( \pm[p]) .
$$

On the other hand, $\operatorname{elm}_{p, l}^{ \pm}$induce the same birational $\mathbb{P}^{1}$-bundle transformation

$$
\operatorname{elm}_{p, l}: P=\mathbb{P}(E) \rightarrow P^{\prime}
$$

since $\phi^{+}$and $\phi^{-}$coincide both in $\operatorname{PGL}\left(2, \mathcal{O}\left(U^{*}\right)\right)$ and $\operatorname{PGL}(2, \mathcal{M}(U))$.

One still has to verify that our construction only depends on the "parabolic structure" $(p, l)$, not on the choice of the local trivialization $Y$. For another choice

$$
\tilde{Y}=M \cdot Y, \quad M \in \mathrm{GL}(2, \mathcal{O}(U)),
$$

one has to check that $\phi^{+}(\tilde{Y})=\phi^{+}(M \cdot Y)=\tilde{M} \cdot \phi^{+}(Y)$ with $\tilde{M} \in$ $\mathrm{GL}(2, \mathcal{O}(U))$. Indeed, if $M=\left(\begin{array}{ll}a & b \\ c & d\end{array}\right)$, then $\tilde{M}=\left(\begin{array}{cc}a & b / x \\ x c & d\end{array}\right)$; since $l$ has to be spanned by $\tilde{Y}=\left(\begin{array}{l}0 \\ 1\end{array}\right)$, we have $b(0)=0$ and $\tilde{M}$ is holomorphic with $\operatorname{det}(\tilde{M})=\operatorname{det}(M) \neq 0$.

A similar computation shows that the line $l^{ \pm} \subset E_{p}^{ \pm}$, defined by $\phi^{ \pm}=$ $\left(\begin{array}{l}1 \\ 0\end{array}\right)$ in the construction above, does not depend on our choices. In other word, given a bundle $E$ equipped with a parabolic structure over $p, l \subset E_{p}$, elementary transformations define a birational tranformation

$$
\operatorname{elm}_{p}^{ \pm}:(E, l) \rightarrow\left(E^{ \pm}, l^{ \pm}\right)
$$


between parabolic bundles which is well defined up to left-and-right composition by parabolic bundle isomorphisms. It also follows from computations above that

$$
\operatorname{elm}_{p}^{ \pm} \circ \operatorname{elm}_{p}^{\mp}:(E, l) \rightarrow\left(E^{\prime}, l^{\prime}\right)
$$

are parabolic bundle isomorphisms. In this sense, $\operatorname{elm}_{p}^{+}$and $\operatorname{elm}_{p}^{-}$are inverse to each other. We can also consider a general rank 2 parabolic bundle $(E, l)$ over $(X, S)$ where $S \subset X$ is a finite subset, and $l: S \rightarrow \mathbb{P}\left(\left.E\right|_{S}\right)$ a section of the projective bundle induced over $S$. The elementary transformations $\operatorname{elm}_{p}^{ \pm}:(E, l) \rightarrow\left(E^{ \pm}, l^{ \pm}\right)$are defined between parabolic bundles over $(X, S)$ like above when $p \in S$ (note that $\operatorname{elm}_{p, l(p)}^{ \pm}$induces an isomorphism of parabolic bundles over $\left.\left(X^{*}, S^{*}\right)\right)$ and as the identity when $p \notin S$. Finally, if $p_{1}, p_{2} \in S$ are two distinct points, elementary transformations $\operatorname{elm}_{p_{1}}^{ \pm}$and $\operatorname{elm}_{p_{2}}^{ \pm}$commute (up to parabolic bundle isomorphisms) so that one can define elm $S_{S^{\prime}}^{ \pm}$for any subset $S^{\prime} \subset S$.

Now, we would like to describe how elementary transformations act on parabolic connections $(E, \nabla, l):(E, l)$ is a parabolic bundle over $(X, S)$ like above and $\nabla$ a meromorphic connection on $E$. Let $p \in S$ and denote by $\nabla^{ \pm}$the push-forward of $\nabla$ by the elementary transformation $\operatorname{elm}_{p}^{ \pm}:(E, l) \rightarrow\left(E^{ \pm}, l^{ \pm}\right): \nabla^{ \pm}$is a meromorphic connection on $E^{ \pm}$. Under notations above, if $\nabla$ is defined in coordinates $(x, Y)$ by

$$
Y \mapsto d Y-\Omega Y, \quad \Omega=\left(\begin{array}{ll}
\alpha & \beta \\
\gamma & \delta
\end{array}\right)
$$

then $\nabla^{ \pm}$is defined in the $E^{ \pm}$local trivialization $Y^{ \pm}=\phi^{ \pm}(Y)$ by $Y^{ \pm} \mapsto$ $d Y^{ \pm}-\Omega^{ \pm} Y \pm$ where

$$
\Omega^{+}=\left(\begin{array}{cc}
\alpha & \frac{\beta}{x} \\
x \gamma & \delta+\frac{d x}{x}
\end{array}\right) \text { and } \Omega^{-}=\left(\begin{array}{cc}
\alpha-\frac{d x}{x} & \frac{\beta}{x} \\
x \gamma & \delta
\end{array}\right) .
$$

If $p$ is not a pole of $\nabla$, then $\nabla^{ \pm}$has a logarithmic pole at $p$. If $p$ is a pole of order $k$ of $\nabla$, there are two cases:

- if $l(p)$ is an eigenvector of $\nabla$ at $p$ (i.e. of $x^{k} \Omega$ at $x=0$ ), then $\nabla^{ \pm}$has a pole of order $k$ or $k-1$ at $p$;

- if not, then $\nabla^{ \pm}$has a pole of order $k+1$ at $p$.

In any case, $l^{ \pm}(p)$ is an eigenvector of $\nabla^{ \pm}$at $p$.

When $\nabla$ is a logarithmic connection, then $\nabla^{ \pm}$is also logarithmic if, and only if, either $p$ is regular, or $p$ is a pole and $l(p)$, an eigenline of $\nabla$. One can then choose the coordinate $Y$ such that $\nabla$ is defined by 


$$
\begin{gathered}
Y \mapsto d Y-A Y \frac{d x}{x} \text { with } \\
\Omega=\left(\begin{array}{cc}
\theta_{1} \frac{d x}{x} & 0 \\
0 & \theta_{2} \frac{d x}{x}
\end{array}\right), \quad\left(\begin{array}{cc}
(\theta+n) \frac{d x}{x} & x^{n} \frac{d x}{x} \\
0 & \theta \frac{d x}{x}
\end{array}\right) \quad \text { or } \quad\left(\begin{array}{cc}
\theta \frac{d x}{x} & 0 \\
x^{n} \frac{d x}{x} & (\theta+n) \frac{d x}{x}
\end{array}\right) \\
A=\left(\begin{array}{cc}
\theta_{1} & 0 \\
0 & \theta_{2}
\end{array}\right), \quad\left(\begin{array}{cc}
(\theta+n) & x^{n} \\
0 & \theta
\end{array}\right) \text { or } \quad\left(\begin{array}{cc}
\theta & 0 \\
x^{n} & (\theta+n)
\end{array}\right) .
\end{gathered}
$$

(including the regular case $A=0$ ) with the restriction $n>0$ in the middle case. Then $\nabla^{ \pm}$is given in coordinate $Y^{ \pm}=\phi^{ \pm}(Y)$ by $Y^{ \pm} \mapsto$ $d Y^{ \pm}-A^{ \pm} Y^{ \pm} \frac{d x}{x}$ with respectively

$$
A^{+}=\left(\begin{array}{cc}
\theta_{1} & 0 \\
0 & \theta_{2}+1
\end{array}\right), \quad\left(\begin{array}{cc}
(\theta+n) & x^{n-1} \\
0 & \theta+1
\end{array}\right) \quad \text { or } \quad\left(\begin{array}{cc}
\theta & 0 \\
x^{n+1} & (\theta+n+1)
\end{array}\right)
$$

and

$$
A^{-}=\left(\begin{array}{cc}
\theta_{1}-1 & 0 \\
0 & \theta_{2}
\end{array}\right), \quad\left(\begin{array}{cc}
(\theta+n-1) & x^{n-1} \\
0 & \theta
\end{array}\right) \quad \text { or } \quad\left(\begin{array}{cc}
\theta-1 & 0 \\
x^{n+1} & (\theta+n)
\end{array}\right) \text {. }
$$

We resume:

- if $p$ is a regular point of $\nabla$, i.e. $A=0$, then $\nabla^{ \pm}$is logarithmic with eigenvalues 0 and \pm 1 ;

- if $p$ is a pole and $\theta$ an eigenvalue of $\nabla$ at $p$, there exists one and only one eigenline $l(p)$ associate to $\theta$ except in the diagonal case when $\theta_{1}=\theta_{2}=\theta$; when $\nabla$ is trace free, this later case does not occur and (the eigenspace of) each eigenvalue defines a parabolic structure over $p$;

- if $\left\{\theta_{1}, \theta_{2}\right\}$ denote the eigenvalues and if $l(p)$ is the eigenline associate to $\theta_{2}$, then $\nabla^{+}$(resp. $\nabla^{-}$) has eigenvalues $\left\{\theta_{1}, \theta_{2}+1\right\}$ (resp. $\left.\left\{\theta_{1}-1, \theta_{2}\right\}\right) ; \nabla^{ \pm}$are of diagonal type if, and only if, $\nabla$ is; the parabolic structure of $\left(E^{ \pm}, \nabla^{ \pm}, l^{ \pm}\right)$over $p$ corresponds to the eigenvalue $\theta_{1}$.

The trace of the connection is changed by

$$
\operatorname{tr}\left(\nabla^{ \pm}\right)=\operatorname{tr}(\nabla) \otimes \zeta^{ \pm}
$$

where $\zeta$ is the unique logarithmic connection on $\mathcal{O}_{X}( \pm[p])$ having a single pole at $p$ with residue \pm 1 and trivial monodromy. Indeed, the monodromy does not change by a birational bundle transformation.

\section{REFERENCES}

[1] D. Arinkin and S. Lysenko, Isomorphisms between moduli spaces of SL(2)bundles with connections on $\mathbb{P}^{1} \backslash\left\{x_{1},, x_{4}\right\}$, Math. Res. Lett. 4 (1997) 181-190.

[2] M. F. Atiyah, Vector bundles over an elliptic curve, Proc. London Math. Soc. (3) 7 (1957) 414-452.

[3] A. Beilinson and V. Drinfeld, Opers, arXiv:math/0501398 [math.AG]. 
[4] P. Boalch, From Klein to Painlevé via Fourier, Laplace and Jimbo, Proc. London Math. Soc. (3) 90 (2005) 167-208.

[5] A.A. Bolibrukh, The Riemann-Hilbert problem, Russian Math. Surveys 45 (1990) 1-58.

[6] M. Brunella, Birational geometry of foliations, Monografías de Matemática. Instituto de Matemática Pura e Aplicada (IMPA), Rio de Janeiro, 2000.

[7] S. Cantat and F. Loray, Dynamics on character varieties and Malgrange irreducibility of Painlevé VI equation, Ann. Inst. Fourier (Grenoble) 59 (2009) 2927-2978.

[8] D. Cerveau, A. Lins Neto, F. Loray, J. V. Pereira and F. Touzet, Complex codimension one singular foliations and Godbillon-Vey sequences, Mosc. Math. J. 7 (2007) 21-54.

[9] R.C. Churchill, Two generator subgroups of $\mathrm{SL}(2, \mathbf{C})$ and the hypergeometric, Riemann, and Lam equations. Differential algebra and differential equations, J. Symbolic Comput. 28 (1999) 521-545.

[10] P. Deligne, Équations différentielles à points singuliers réguliers, Lecture Notes in Mathematics 163. Springer-Verlag, Berlin-New York, 1970.

[11] H. Esnault and E. Viehweg, Semistable bundles on curves and irreducible representations of the fundamental group, in Algebraic Geometry, Hirzebruch 70 (Warsaw, 1998), Contemp. Math., Vol. 241, Amer. Math. Soc., Providence, RI, (1999), 129-138.

[12] D. Gallo, M. Kapovich and A. Marden, The monodromy groups of Schwarzian equations on closed Riemann surfaces, Annals of Math. 151 (2000) 625-7004.

[13] W. M. Goldman - The modular group action on real SL(2)-characters of a one-holed torus, Geometry and Topology 7 (2003) 443-486.

[14] R. C. Gunning, Special Coordinate Coverings of Riemann Surfaces, Math. Annalen 170 (1967) 67-86.

[15] R. Hartshorne, Algebraic geometry, Graduate Texts in Mathematics, No. 52. Springer-Verlag, New York-Heidelberg, 1977.

[16] V. Heu, Stability of rank 2 vector bundles along isomonodromic deformations, Math. Ann. 344 (2009) 463-490.

[17] N.J. Hitchin, Twistor spaces, Einstein metrics and isomonodromic deformations, J. Differential Geom. 42 (1995) 30-112.

[18] A. T. Huckleberry and G. A. Margulis, Invariant analytic hypersurfaces, Invent. Math. 71 (1983) 235-240.

[19] M. Inaba, K. Iwasaki and M. Saito, Dynamics of the Sixth Painlevé equation, Théories asymptotiques et équations de Painlevé, 103-167, Sémin. Congr., 14, Soc. Math. France, Paris, 2006.

[20] E.L. Ince, Ordinary Differential Equations. Dover Publications, New York, 1944.

[21] S. Kawai, Isomonodromic deformation of fuchsian projective connections on elliptic curves, Nagoya Math. J. 171 (2003) 127-161.

[22] A. M. Levin and M. A. Olshanetsky, Painlevé-Calogero correspondence, Calogero-Moser-Sutherland models (Montréal, QC, 1997), 313-332, CRM Ser. Math. Phys., Springer, New York, 2000.

[23] F. Loray, Okamoto symmetry of Painlevé VI equation and isomonodromic deformation of Lamé connections, Algebraic, analytic and geometric aspects of 
complex differential equations and their deformations. Painlevé hierarchies. 129136, RIMS Kôkyûroku Bessatsu, B2, Res. Inst. Math. Sci. (RIMS), Kyoto, 2007.

[24] F. Loray and J. V. Pereira, Transversely projective foliations on surfaces: existence of minimal form and prescription of monodromy, Internat. J. Math. 18 (2007) 723-747.

[25] F. Loray, M. van der Put and F. Ulmer, The Lamé family of connections on the projective line, Ann. Fac. Sci. Toulouse 17 (2008) 371-409.

[26] F. Loray, M.-H. Saito and C. Simpson, Foliations on the moduli space of rank two connections on the projective line minus four points, Geometric and differential Galois theories, 117-170, Sémin. Congr., 27, Soc. Math. France, Paris, 2013.

[27] B. Malgrange, Sur les déformations isomonodromiques I. Singularités régulières, Mathematics and physics (Paris, 1979/1982), 401-426, Progr. Math., 37, Birkhäuser Boston, Boston, MA, 1983.

[28] Yu. I. Manin, Sixth Painlevé equation, universal elliptic curve, and Mirror of $\mathbb{P}^{2}$, Amer. Math. Soc. Transl. (2) 186 (1998) 131-151.

[29] M. Mazzocco, Picard and Chazy solutions to the Painlevé VI equation, Math. Ann. 321 (2001) 157-195.

[30] M. Noumi and Y. Yamada, A new Lax pair for the sixth Painlevé equation associated with $\widehat{s o}(8)$, Microlocal analysis and complex Fourier analysis, 238252, World Sci. Publishing, River Edge, NJ, 2002.

[31] D. Novikov and S. Yakovenko, Lectures on meromorphic flat connections in Normal forms, bifurcations and finiteness problems in differential equations, 387-430, NATO Sci. Ser. II Math. Phys. Chem., 137, Kluwer Acad. Publ., Dordrecht, 2004.

[32] K. Okamoto, Sur les feuilletages associés aux équations du second ordre à points critiques fixes de P. Painlevé, Japan J. Math. 5 (1979) 1-79.

[33] K. Okamoto, Studies on the Painlevé equations I. Sixth Painlevé equation $P_{\mathrm{VI}}$, Ann. Mat. Pura Appl. (4) 146 (1987) 337-381.

[34] J. Palmer, Zeroes of the Jimbo-Miwa-Ueno tau function, J. Math. Phys., 40 12 (1999) 6638-6681.

[35] É. Picard, Mémoire sur la théorie des fonctions algébriques de deux variables, Jour. Math. Pures et Appl. 5 (1889) 135-319.

[36] H. Poincaré, Sur les groupes des équations linéaires, Acta Mathematica 4 (1884) 201-311.

[37] H. Sakai, Rational surfaces associated with affine root systems and geometry of the Painlevé equations, Comm. Math. Phys. 220 (2001) 165-229.

[38] M.-H. Saito, T. Takebe, H. Terajima, Deformation of Okamoto-Painlevé pairs and Painlevé equations, J. Algebraic Geom. 11 (2002) 311-362.

[39] L. W. Tu, Semistable bundles over an elliptic curve, Adv. Math. 98 (1993) $1-26$.

[40] A. Weil, Généralistion des fonctions abéliennes, J. Math. Pures Appl. 17 (1938) 47-87.

[41] A. Zotov, Elliptic linear problem for the Calogero-Inozemtsev model and Painlevé VI equation, Lett. Math. Phys. 67 (2004) 153-165. 
Frank LORAY (Directeur DE REChERCHES Au CNRS) IRMAR, Campus de Beaulieu, 35042 Rennes Cedex (France)

E-mail address: frank. loray@univ-rennes1.fr 